\title{
Many-body Correlation Effects in the Ultrafast Nonlinear Optical Response of Confined Fermi Seas
}

\author{
I. E. Perakis \& T. V. Shahbazyan \\ Department of Physics \& Astronomy \\ Vanderbilt University \\ Nashville, TN 37235
}

November 3, 2018

\begin{abstract}
The dynamics of electrons and atoms interacting with intense and ultrashort optical pulses presents an important problem in physics that cuts across different materials such as semiconductors and metals. The currently available laser pulses, as short as $5 \mathrm{fs}$, provide a time resolution shorter than the dephasing and relaxation times in many materials. This allows for a systematic study of many-body effects using nonlinear optical spectroscopy. In this review article, we discuss the role of Coulomb correlations in the ultrafast dynamics of modulation-doped quantum wells and metal nanoparticles. We focus in particular on the manifestations of non-Markovian memory effects induced by strong electron-hole and electron-plasmon correlations.
\end{abstract}




\section{Contents}

List of acronyms 4

$1 \quad$ Introduction and Scope of this Review 4

2 Nonlinear Optical Dynamics in Modulation-doped Quantum $\begin{array}{ll}\text { Wells } & 10\end{array}$

$2.1 \quad$ Fermi Edge Singularity in Linear Absorption . . . . . . . . . . 10

2.2 vitrafast Dynamics . . . . . . . . . . . . . . . . . . . 12

3 Nonlinear Optical Dynamics in Metal Nanoparticles 22

3.1 Surface Plasmon Resonance in Linear absorption . . . . . . . . 23

3.2 vltrafast Dynamics . . . . . . . . . . . . . . . . . . 24

4 Coherent Ultrafast Response of the Fermi Edge Singularity:

Formalism 30

4.1 Basic equations $\ldots \ldots \ldots$. . . . . . . . . . . . . . . . . . . . 30

4.2 Overview of the Coupled Cluster Expansion . . . . . . . . . . 34

$4.3 \quad$ Effective Hamiltonian and Transition Matrix Elements . . . . 35

4.3 .1 Discussion for Doped Semiconductors . . . . . . . . . . 35

4.3 .2 Second Quantization Expressions . . . . . . . . . . . . 37

4.4 Description of the Electron-Hole Pair Dynamics . . . . . . . . 40

4.5 The Case of Two Coupled Subbands . . . . . . . . . . . . . . 43

5 Coherent Ultrafast Dynamics of the Fermi Edge Singularity 46

5.1 Discussion of the Physics: Monochromatic Excitation . . . . . 46

5.2 Results for Short-pulse Excitation . . . . . . . . . . . . . . 47

5.3 Ultrafast Dynamics of the FES-Exciton Hybrid . . . . . . . . 52

6 Quasiparticle Scattering with Surface Collective Modes in Metal Nanoparticles $\quad 55$

$6.1 \quad$ Electron-Electron Interactions in Metal Nanoparticles . . . . . 55

6.2 Conduction Electron Scattering . . . . . . . . . . . . . . . 59

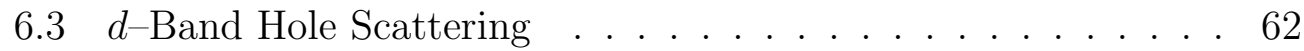

\begin{tabular}{|lll}
\hline 7 & Surface Plasmon Nonlinear Optical dynamics & 64
\end{tabular}

\begin{tabular}{|ll}
\hline 8 Conclusions & 68
\end{tabular} 
$\begin{array}{ll}\text { Acknowledgements } & \mathbf{7 0}\end{array}$

\begin{tabular}{ll}
\hline A APPENDIX A & 70
\end{tabular}

\begin{tabular}{ll}
\hline B APPENDIX B & 77
\end{tabular}

\begin{tabular}{lll}
\hline C APPENDIX 9 & 78 \\
\hline
\end{tabular}

$\begin{array}{ll}\text { References } & 79\end{array}$ 


\section{List of acronyms}

$\begin{array}{ll}\text { FES } & \text { Fermi Edge Singularity } \\ \text { FS } & \text { Fermi sea } \\ e-h & \text { Electron-hole } \\ e-e & \text { Electron-electron } \\ h-h & \text { Hole-hole } \\ e-p & \text { Electron-phonon } \\ h-p & \text { Hole-phonon } \\ \text { HFA } & \text { Hartree-Fock approximation } \\ \text { SBE's } & \text { Semiconductor Bloch Equations } \\ \text { SP } & \text { Surface Plasmon } \\ \text { FWM } & \text { Four Wave Mixing } \\ \text { PP } & \text { Pump-probe } \\ \text { RPA } & \text { Random Phase Approximation } \\ \text { QW } & \text { Quantum Well } \\ \text { CCE } & \text { Coupled Cluster Expansion } \\ \text { fs } & \text { Femtosecond } \\ \text { ps } & \text { Picosecond } \\ \text { MDQW } & \text { Modulation-doped Quantum Well } \\ \text { TI-FWM } & \text { Time-integrated Four Wave Mixing }\end{array}$

\section{Introduction and Scope of this Review}

One of the most exciting thrusts in Physics focusses on understanding the properties of systems possessing many degrees of freedom. The goal is to relate such properties to the interactions among the elementary excitations. Such many-body problems have been a driving force in many different subfields of Physics and Chemistry: condensed matter physics, nuclear physics, quantum chemistry, high energy physics, $\cdots$ In condensed matter physics, the study of many-body phenomena has led to many exciting discoveries: superconductivity, quantum Hall effect, Kondo effect, Mott transition, quantum phase transitions, Fermi Edge and X-ray Edge Singularities, $\cdots$. In most cases, such fascinating phenomena have been observed in equilibrium systems at low temperatures. In comparison, the role of many-body effects in the dynamics of non-equilibrium many-electron systems presents a less explored frontier. 
Ultrafast nonlinear optical spectroscopy provides unique and powerful tools for studying the dynamics of many-body correlations. In such experiments, an intense optical pulse, the pump, with duration shorter than the scattering time or the period of the elementary excitations photoexcites the system from its ground state. The subsequent dynamics is then monitored by using a second pulse, the probe. By measuring the amplitude and the phase of the optical field emerging from the photoexcited sample, one can gain valuable insight into the interplay between many-body and quantum confinement effects during very short time scales. A good understanding of the role of such fundamental physics can lead to new ideas regarding the next generation of photonic and opto-electronic devices. These operate under intense excitation conditions in the technologically important Tbit/sec regime and are often based on semiconductor heterostructures and novel lowdimensional structures. Such considerations have spurred intense efforts in recent years aimed at understanding how to control the optical dynamics during sub-picosecond time scales $\left(1 \mathrm{Tbit} / \mathrm{sec}=(1 \mathrm{ps})^{-1}\right)$. In the highly non-equilibrium femtosecond regime, the dominant effects on the nonlinear optical dynamics come from the Pauli exclusion principle (Phase Space Filling effects) and the Coulomb interactions among the optically-excited and Fermi sea carriers. The phonons also play an important role, especially during picosecond time scales.

Following their photoexcitation, the electrons and holes undergo a number of different relaxation stages as they scatter among themselves via the Coulomb interaction and with the phonons via the electron-phonon $(e-p)$ interaction. Below we briefly discuss each of these relaxation stages. Initially (stage I, the coherent regime) the photoexcited electrons and holes have a well-defined phase relationship: since the photon momentum is negligible, they are restricted to have opposite momenta. Such a coherence is described by an optical polarization. In the very beginning, the photoexcited carriers interact via the bare Coulomb interaction. After time intervals of the order of the inverse plasmon frequency corresponding to the carrier density, the interactions become screened. The problem can then be approached in terms of interacting Fermi liquid quasi-particles [1]. Even though in typical metals such screening occurs within an extremely short time interval $\sim 1$ fs, in semiconductors the inverse plasma frequency is of the order of tens of femtoseconds and therefore the above regime is accessible experimentally by using $\sim 5-10$ fs optical pulses. If one tunes the pump frequency above the onset of linear absorption, real carriers are photoexcited, in which case 
the duration of stage $\mathbf{I}$ is determined by the dephasing time, $T_{2}$. In metals, the latter is typically of the order of a few femtoseconds, while in semiconductors it can be as long as a few picoseconds. The dephasing processes are suppressed if one tunes the pump frequency below the onset of linear absorption. Under such below-resonance excitation conditions, the coherent effects become dominant since only virtual carriers are photoexcited and thus dissipative scattering processes cannot occur. The time-dependence of the optical polarization is then determined by the pulse duration, which leads to extremely fast nonlinearities.

Immediately after the $e-h$ phase coherence is lost, incoherent populations of electrons and holes are formed. These are initially described by nonthermal density distributions. Subsequently, these evolve into hot thermal distributions characterized by an electron temperature that can far exceed that of the lattice. Such a time evolution (stage II) as the carriers equilibrate among themselves is mainly induced by the $e-e, e-h, h-h$, and $e-p$ interactions. The duration of this stage II is determined by the population relaxation time, $T_{1}$, which can be of the order of picoseconds. Subsequently, the $e-p$ interaction leads to the equilibration of the electronic and lattice temperatures on a time scale of tens of picoseconds, followed by the return to thermodynamic equilibrium after hundreds of picoseconds via the recombination of the photoexcited carriers or via the transport of heat to the surroundings (stage III).

A large body of experimental and theoretical work has focussed on different aspects of the above relaxation. Such developments in undoped semiconductors have been extensively described in a number of excellent review articles and books. Among the earlier reviews of interaction effects in highlyexcited undoped semiconductors, we note those of Haug and Schmitt-Rink [2], Stahl and Baslev [3], Zimmermann [4], Schmitt-Rink et. al. [5], and Haug and Koch [6]. More recent reviews focussing on the femtosecond coherent dynamics in undoped semiconductors include those by Mukamel [7], Binder and Koch [8], Shah [9], Haug and Jauho [10], Axt and Mukamel [11], and Chemla [12]. We also note the collections of papers in Refs. [13, 14, 15, 16]. More recently, there has been a lot of interest in using ultrafast nonlinear spectroscopy to study the dynamics of many-body effects in metals. The charge carrier dynamics and the coherent ultrafast phenomena in bulk metals, metal interfaces, and metal surfaces were recently reviewed by Petek and Ogawa [17.

The purpose of this review article is to provide a comprehensive picture 
of recent experimental and theoretical advances in understanding femtosecond many-body phenomena in Fermi sea systems. As has been extensively discussed in the review articles and books listed above, even though a description of the ultrafast dynamics based on few-level models [18, 19, 20] has been very successful in atomic and molecular systems, it faces serious shortcomings when applied to semiconductors and metals. The development of microscopic non-equilibrium many-body theories has been found to be necessary for describing the femtosecond dynamics and has made this field an exciting area of research in condensed matter physics. In view of the large body of work, especially in undoped semiconductors, we will concentrate here on specific aspects of the many-body physics that have not been extensively reviewed elsewhere. In particular, we will not touch on the impressive advances in undoped semiconductors and will attempt to provide a unified picture of the ultrafast dynamics in different confined Fermi sea systems. We will mainly focus on coherent effects due to the Coulomb interaction and will only briefly discuss the electron-phonon $(e-p)$ interaction effects. The main body of this article discusses the recent breakthroughs in understanding the sub-picosecond dynamics in modulation-doped quantum wells (MDQW's) and metal nanoparticles, with particular emphasis on non-Markovian memory effects induced by carrier-carrier many-body correlations. Such dynamical effects are beyond the scope of the dephasing and relaxation time approximations. One should note here that a detailed quantitative description of the experimental results presents a formidable task in the subpicosecond regime, which requires sophisticated numerical simulations as well as different approximations. This can make it difficult to discuss in a simple and intuitive way the important many-body physics conveyed by the experiment. Here we will focus on the simplest possible models that can capture the most important physics associated with the non-equilibrium Coulomb correlations and attempt to provide, to the degree possible, an intuitive explanation of the often delicate experimental features.

Let us now briefly discuss how many-body correlations enter into the ultrafast nonlinear optical response. The most commonly used theoretical approach starts with the Heisenberg equations of motion of the one-particle density matrix, $\rho_{i j}(t)$, where the indices $i, j=1,2$ label the conduction and valence band [7]. The off-diagonal density matrix element, $\rho_{12}(t)=P(t)$, is the optical polarization, while the diagonal matrix elements, $\rho_{i i}(t)$, correspond to the electron and hole occupation numbers (density distributions). Such equations of motion must be solved to obtain the polarization, which 
in turn acts as the source term in the Maxwell equations that determine the signal measured in the experiment [7]. The difficulty in treating the many-body interactions comes from the coupling of the one-particle density matrix to many-particle correlation functions (higher density matrices). As is typical for many-body systems, this leads to an infinite hierarchy of coupled equations which cannot be solved exactly [7, 11. One must therefore devise a controlled truncation scheme. One possibility is to adopt the time-dependent Hartree-Fock approximation (HFA), which relies on the factorization of the many-particle correlation functions and only includes the two-particle interaction effects. Such an approach developed into the very successful Semiconductor Bloch Equations (SBE's) formalism (see e.g. Refs. 21, 22, 23, 24, 25, 6]). However, many recent experimental observations, reviewed e.g. in Ref. [12], could not be explained within such a mean field approach and were attributed to four-particle and higher order Coulomb correlations. In order to develop a many-body theory that can capture such effects, it was noted by several different groups that, in undoped semiconductors, the above infinite density matrix hierarchy truncates if one adopts an expansion in terms of the optical fields [11, 26, 27, 28, 29, 7, 8]. This is due to the fact that, in the undoped semiconductor ground state, the conduction band is empty while the valence band is full. Such a method for treating the Coulomb correlations nonperturbatively is often referred to as the "dynamic controlled truncation scheme". An alternative many-body approach has been based on diagrammatic expansions for Keldysh Green's functions, whose multiple-time dependence is typically eliminated by using the Kadanoff-Baym ansatz [10].

Let us now discuss how non-Markovian memory effects enter into the density matrix equations of motion. Due to the many degrees of freedom that affect the dynamics, one usually distinguishes between a subsystem interacting directly with the optical fields (e.g. the photoexcited $e-h$ pair) and a reservoir/bath consisting of all the other degrees of freedom (e.g. the phonons or the Fermi sea excitations). The polarization equation of motion can be cast in the form

$$
\frac{\partial}{\partial t} P(t)=\left.\frac{\partial}{\partial t} P(t)\right|_{\mathrm{coh}}+\left.\frac{\partial}{\partial t} P(t)\right|_{\mathrm{scatt}}
$$

where the first term describes the Hartree-Fock interactions among the coherent $e-h$ pairs, while the second term describes the many-body scattering processes among the carriers as well as between the carriers and the bath 
excitations. The latter term can be expressed in the general form

$$
\left.\frac{\partial}{\partial t} P(t)\right|_{\text {scatt }}=\int_{-\infty}^{t} d t^{\prime} \Gamma\left(t, t^{\prime}\right) P\left(t^{\prime}\right)
$$

where $\Gamma\left(t, t^{\prime}\right)$ is a memory kernel (similar equations can also be written for the carrier distribution functions). The importance of the many-body correlations of interest here is that they determine the dependence of $\Gamma\left(t, t^{\prime}\right)$ on the two times $t$ and $t^{\prime}$ and therefore govern the memory effects. In systems where only times $t^{\prime} \sim t$ contribute to the above integral, one can apply the Markovian approximation, in which case

$$
\left.\frac{\partial}{\partial t} P(t)\right|_{\text {scatt }} \sim \Gamma(t) P(t)
$$

In many cases, one can further approximate $\Gamma(t)$ by a time-independent constant, the dephasing energy width $\sim 1 / T_{2}$. For example, such an approximation allows one to include, in a semi-phenomenological way, the effect of the scattering processes in the SBE's. However, when such a Markovian approximation breaks down, the many-body correlations can affect the qualitative ultrafast dynamics by inducing strong memory effects and non-exponential polarization decay. Such effects in undoped semiconductors are known to arise e.g. from exciton-exciton correlations or from $e-p$ and carrier-carrier interactions in the quantum kinetic regime and have been extensively reviewed e.g. in Refs. [12, 10]. Here we focuss on analogous effects whose origin lies in the non-perturbative Coulomb correlations between the photoexcited carriers and the cold electron Fermi sea in MDQW's and small metal nanoparticles.

This review is organized as follows. First we present an overview of the experimental results on the ultrafast nonlinear optical dynamics in MDQW's (Section 2) and metal nanoparticles (Section 3). In Section 1 we outline the formalism necessary for describing the coherent ultrafast nonlinear optical response of Fermi sea systems. In Section 5, the ultrafast nonlinear dynamics of the Fermi Edge Singularity (FES) in the coherent regime is discussed. In Section 6 the size-dependent dynamical screening of the Coulomb interaction in metal nanoparticles is discussed and the results for the quasiparticle scattering rates are reviewed. In Section 0 these results are used to describe the ultrafast surface plasmon (SP) dynamics in small noble-metal nanoparticles. Section 8 concludes the review. 


\section{Nonlinear Optical Dynamics in Modulation- doped Quantum Wells}

By separating the ionized impurity donors from the electrons, modulation doping can produce a high mobility two-dimensional electron gas. In contrast to the case of a photoexcited electron gas, the temperature of such a system can be lowered, which enables the observation of fascinating many-body phenomena such as the Quantum Hall Effect [30, 31, 32, 33] and the Fermi Edge Singularity [5].

Recently there has been a lot of interest in studying the ultrafast dynamics in MDQW's. The role of the interactions between the photoexcited and the Fermi sea (FS) carriers during the thermalization stage II has been reviewed e.g. in Refs. [9, 14, 10] and is only briefly discussed here. In the section 3.2 we will review corresponding measurements in metal films and nanoparticles and compare with MDQW's. Much less is known about the role of many-body effects in the coherent regime (stage I). Even though some aspects of the effects of the $e-e$ scattering have been described within the dephasing time approximation, the role of the $e-h$ correlations between the photoexcited holes and the FS electrons has only recently been studied. The main difficulty in treating such $e-h$ correlation effects comes from the nonperturbative nature of the Fermi edge singularity (FES), which dominates the absorption spectrum close to the absorption onset, and which cannot be described within the dephasing time approximation. Important here is that the $e-h$ interactions between a heavy photoexcited hole and the FS-electrons lead to the scattering of a macroscopic number of low-energy FS-pairs, which readjusts the entire FS into a new orthogonal scattering state in the course of the optical excitation (Anderson orthogonality catastrophe [34]). Before we discuss the ultrafast dynamics due to such effects, we briefly summarize in the next section the linear optical properties of MDQW's.

\subsection{Fermi Edge Singularity in Linear Absorption}

Close to the onset of linear absorption and for low-temperatures, the optical properties of MDQW's are dominated by the FES. The latter is a many-body resonance that has been observed in doped semiconductors (see e.g [35, 36, 37, 38, 39, 5]) as well as in metals [40, 41], where it is referred to as the $\mathrm{X}-$ ray Edge Singularity. Despite the screening of the Coulomb interaction, the 
strength of this resonance is comparable to that of the undoped QW excitons. The non-Lorentzian lineshape of the FES can be viewed as originating from the decay of an excitonic bound state caused by its interactions with the gapless FS excitations [42]. In the case of holes localized due to e.g. the disorder [35] or for core holes in metals, the lineshape of the FES close to the onset of absorption can be approximately described by using the analytic power law expression 42, 43, 44, 5]

$$
\alpha(\omega) \propto \mathcal{N}\left(\frac{E_{F}}{\omega}\right)^{\beta},
$$

where $\mathcal{N}$ is the density of states, $E_{F}$ is the Fermi energy, $\omega$ is the optical frequency measured from the Fermi edge, and $\beta=2 \delta / \pi-(\delta / \pi)^{2}$ is the FES exponent, where $\delta \sim \tan ^{-1}(\pi g)$ is the $\mathrm{s}^{-}$wave phaseshift of the screened $e-h$ potential $V$ evaluated at $E_{F}$, and $g=V \mathcal{N}$ is the dimensionless parameter characterizing the $e-h$ scattering strength. Such a non-Lorentzian lineshape results from the competition between the Mahan singularity, due to the attractive interaction between the FS and the localized hole (vertex correction effect, [45, 46]) and the Anderson orthogonality catastrophe due to the readjustment of the FS density profile during the optical transition (hole self-energy effect, [34]). In the case of finite hole mass $m_{h}$, the FES is broadened by an additional energy width of the order of the hole recoil energy $\sim m_{e} / m_{h} E_{F}$, where $m_{e}$ is the electron mass 42, 47, 48].

In a first approximation, the excitonic effects in a MDQW can be described by extending the mean field approach (HFA) to include the effects of the screening and the Pauli blocking due to the FS [5, 42, 49]. However, such a static treatment of the FS leads to a spurious bound state [5, 42] with respect to the Fermi energy, $E_{F}$, referred to in the following as the HFA bound state 45]. This discrete state, with binding energy $E_{M}$, would appear at the energy $E_{F}-E_{M}$. Obviously, for $E_{M}<E_{F}$ (as in typical MDQW's), such a state cannot exist since it overlaps with the FS continuum, with which it interacts via the $e-h$ potential [5, 42]. The "unbinding" of this HFA bound state occurs via its interactions with the FS excitations, which are not taken into account in the HFA 42]. Note that, in two-dimensional systems, a static FS allows for bound states even for arbitrarily small attractive interactions and therefore such an unbinding cannot arise from static screening or from Pauli blocking effects. This spurious bound state could be artificially merged with the continuum by introducing a dephasing time comparable to its binding energy. Such an approximation, however, neglects competely the dynamical 
correlations between the photoexcited $e-h$ pair and the FS excitations. A microscopic description of the unbinding of the HFA bound state presents a nontrivial problem due to the non-perturbative nature of the $e-h$ correlations between the photoexcited hole and the FS excitations [42]. Even in linear absorption, within Green function techniques, this problem is rather involved because vertex correction diagrams with arbitrarily many crossed $e-h$ interaction lines are as divergent as the ladder diagrams and should be treated on equal footing [43, 46]. To perform such a task, one must sum up at least the parquet diagrams and address the three-body correlations between the photoexcited hole and a FS excitation [42, 47]. Therefore, alternative methods were developed for the case of linear absorption, based on Fermi's golden rule with many-electron eigenstates expressed in terms of Slater determinants [41]. Such approaches become exact in the limit $m_{e} / m_{h} \ll 1$ and describe quite accurately the FES lineshapes observed in typical MDQW's 41, 50, 48, 51. Another approach to the FES problem is based on the coupled cluster expansion (CCE) [52, 53, 54, 55]. This general many-body technique provided exact results in the limit $m_{e} / m_{h} \ll 1$ [56, 57] and was used to treat the hole recoil correlations in one dimension [58. In the latter case, an exact solution was obtained for $m_{e}=m_{h}$. The CCE has also been used to describe the $e-e$ correlation effects [59, 60]. More importantly, however, this method is well-suited for describing correlations in non-equilibrium systems, where it retains the advantages of diagrammatic expansions without resorting to the Kadanoff-Baym ansatz or to the Markovian approximation [61].

\subsection{Ultrafast Dynamics}

In this section we summarize some recent studies of the dephasing and relaxation processes in MDQW's. The earlier work focussed on the role of the interactions between the FS and the photoexcited carriers on the thermalization during relaxation stage II. In most cases, a non-thermal distribution of real electron and hole carriers was photoexcited well above the Fermi surface and the subsequent incoherent population dynamics was monitored with ultrafast pump-probe spectroscopy. Note that, in such experiments, the measured differential transmission is

$$
D S T(\omega, \tau)=\frac{\Delta T_{s}(\omega, \tau)}{T_{0}(\omega)}=\frac{T_{s}\left(\mathcal{E}_{p}\right)-T_{s}\left(\mathcal{E}_{p}=0\right)}{T_{s}\left(\mathcal{E}_{p}=0\right)},
$$


where $T_{s}\left(\mathcal{E}_{p}\right)$ is the transmission coefficient in the probe direction in the presence of the pump field $\mathcal{E}_{p}$ and $\tau$ denotes the time delay between the pump and probe optical pulses. For samples with sufficiently small thickness $d$ such that $\Delta \alpha d$ is small, the differential transmission reproduces the change, $\Delta \alpha(\omega, \tau)$, in the probe absorption coefficient $\alpha(\omega, \tau)$ which is induced by the pump photoexcitation: $D S T(\omega, \tau) \sim-\Delta \alpha(\omega, \tau) d$.

For a simple intuitive interpretation of the thermalization experiments in MDQW's, it is often assumed that, for free carriers photoexcited well above the onset of absorption and for time scales much longer than the dephasing time, the differential absorption spectrum maps the carrier distribution functions at the probe photon energy at time $\tau$. In particular, the following approximation is often used:

$$
\Delta \alpha(\omega, \tau)=\left(1-f_{e}-f_{h}\right) \alpha(\omega)
$$

where $\alpha(\omega)$ is the linear absorption coefficient and $f_{e}$ and $f_{h}$ are the electron and hole distribution functions at the corresponding energies. Within such an approximation, the time evolution of the differential absorption is determined by the carrier distribution functions at time $\tau$. There have been many theoretical calculations of the distribution function time evolution, mostly based on numerical solutions of the semiclassical Boltzmann equations (see e.g. Refs. 10, 14, 9]). A detailed review of such an approach to thermalization can be found in Ref. [10]. More recently, the dynamics in the quantum kinetic regime and the shortcomings of the Boltzmann equations have been addressed, as reviewed e.g. in Ref. [10].

Knox et. al. [62 studied the effect of the inelastic $e-e$ scattering on the thermalization by photoexciting a non-thermal carrier distribution well above the Fermi surface and then monitoring its time evolution to a thermal distribution at the bottom of the band. They observed that the $e-e$ interactions between the photoexcited and FS electrons significantly enhance the thermalization rates as compared to undoped semiconductors. In particular, the thermalization time in MDQW's was found to be of the order of $\sim 10 \mathrm{fs}$. The differential transmission lineshape mimicked that of a Boltzmann distribution peaked at the bottom of the conduction band, which indicates a non-degenerate carrier distribution. This is expected at room temperatures (as in the experiment of Ref. [62]), where the thermal energy exceeds the Fermi energy, and at high intensities, where the photoexcited carrier density exceeds the FS density. Knox et. al. also observed an instantaneous 
redshift due to the bandgap renormalization by the photoexcited carriers [5]. However, as we discuss later, the situation changes drastically at low temperatures, where the FS electrons have a sharp Fermi-Dirac distribution. Wang et al. 63 performed low temperature pump-probe measurements and found that, at low excitation densities, the differential transmission lineshape corresponded to a redshift of the FES due to the incoherent bandgap renormalization. Importantly, they showed that such a redshift slowly builds up with time as the hot electron temperature decreases due to the cooling to the lattice temperature. This observation points out that the bandgap renormalization depends not only on the carrier density but also on the carrier distribution. At high photoexcited densities, the heating of the electron gas becomes stronger and Wang et. al. observed a bleaching of the FES due to the smearing of the sharp Fermi surface.

Both the above experiments were interpreted in terms of $e-e$ interactions between the photoexcited and the FS carriers. Tomita et. al. 64 performed ultrafast luminescence measurements at low temperatures to investigate the role of the $e-h$ interactions on the thermalization in n-type MDQW's. In particular, they measured the time evolution of the luminescence intensity at the bandgap energy, where the optically-induced perturbation of the electron distribution due to, e.g., heating is minimal. Tomita et. al. found that the number of holes that thermalized to the top of the valence band as a function of time could not accounted for if one assumes a thermalized hole distribution. This result indicates that a substantial fraction of the photoexcited holes are non-thermal for time intervals as long as $\sim 800 \mathrm{fs}$. Furthermore, MonteCarlo simulations 64 indicated that the $e-h$ scattering is the dominant hole thermalization mechanism. Similar measurements in n-doped bulk GaAs were performed by Chebira et. al. (at low temperatures) 65 and Zhou et. al. (at room temperature) [66]. Woerner et. al. [67, 68] studied the hole relaxation in $\mathrm{p}$-doped semiconductors by photoexciting holes from the heavyhole to the split-off valence band and then monitoring the time evolution of the inter-valence-band absorption spectrum by using femtosecond pulses in the mid-infrared region. Due to the absence of conduction electrons, the dynamics is then solely determined by the $h-h$ and $h-p$ scattering. They found that the inter-valence-band scattering via the emission of e.g. optical phonons occurs within a short time interval $<100 \mathrm{fs}$, and that subsequently the phototexcited holes slowly thermalize via $h-h$ scattering with the hole FS within a time interval $\sim 700$ fs.

An important effect of the quantum confinement in QW's is the formation 
of discrete conduction and valence subbands. Recently, there has been much interest in exploring the role of the intersubband excitations in the ultrafast dynamics, partly motivated by device applications such as the quantum cascade laser [69]. Lutgen et. al. [70, 71] performed femtosecond pumpprobe measurements and showed that the intersubband nonlinear absorption is determined by both the intersubband and the intraband electron relaxation. They measured the effects of intersubband pump excitation on the nonlinear absorption due to probe-induced interband transitions from the valence to the conduction band. They observed a population relaxation that proceeds in two stages. First, the electrons photoexcited by the pump from the Fermi sea in the lowest conduction subband relax back via intersubband scattering within a time interval $\sim 1.3 \mathrm{ps}$. The latter is consistent with the intersubband relaxation times due to longitudinal optical phonon emission [72, 73]. This process is followed by intraband $e-e$ relaxation similar to the experiments discussed above. Lutgen et. al. also measured the time evolution of the nonlinear absorption spectrum due to optical transitions between the conduction subbands. They observed an intial decay of the differential transmission within a time interval $\sim 2-3 \mathrm{ps}$, which they attributed to the population relaxation due to the intersubband scattering. This was followed by a slowly decaying differential transmission signal, whose time evolution depended strongly on the photoexcitation frequency (within the linear absorption linewidth). The latter regime was attributed to the intraband population relaxation within the lowest subband as the carriers cool to the lattice temperature.

We now come to the effect of a cold FS on the $e-e$ scattering time. One would expect that the latter should become short as the FS density increases. This is indeed the case for low FS densities, high temperatures, or for large excess energies from the Fermi surface. Hawrylak et. al. [74 obtained the energy-dependent $e-e$ scattering times by calculating the equilibrium self-energies including the effects of the electron-plasmon and $e-p$ interactions. They predicted step-like decreases in the scattering time for electron energy above the Fermi surface that exceeds the onset of plasmon and optical phonon emission. They also concluded that short-range $e-e$ correlations (not included in the RPA) can significantly affect the scattering times at high energies. Such fast $e-e$ scattering of photoexcited electrons well above the Fermi surface can explain for example the absence of spectral hole burning in the pump-probe spectra of MDQW's [62, 9]. However, the situation changes drastically for electron energies close to the Fermi surface and temperatures 
smaller than the Fermi energy. Under such conditions, the $e-e$ scattering is in fact suppressed in the presence of a degenerate FS. Indeed, a photoexcited electron lowers its energy by interacting with a FS electron while the latter scatters above the Fermi surface. For electrons close to the Fermi surface, the drastic reduction of the phase space available for scattering due to the Pauli blocking by the FS electrons as well as the screening leads to the strong suppression of the $e-e$ scattering [1]. This is due to the sharp Fermi-Dirac distribution of the FS electrons at low temperatures. In fact, at zero temperatures, the $e-e$ scattering time $\tau_{e}(E)$ becomes infinite right at the Fermi surface, $E=E_{F}$ [1, 74]. According to Fermi liquid theory [1],

$$
\tau_{e}(E) \propto \frac{E_{F}^{2}}{\left(E-E_{F}\right)^{2}+\left(\pi k_{B} T\right)^{2}},
$$

where $k_{B} T$ is the thermal energy. Note that the above result applies both to metals and MDQW's due to the similar values of $r_{s}$. As the electron temperature increases, the smearing of the Fermi-Dirac distribution allows for the scattering of a photoexcited electron to states below $E_{F}$ and therefore $\tau_{e}(E)$ decreases as compared to the zero temperature limit. Even though the $e-e$ scattering rate initially increases with electron density for low densities, it decreases at higher densities when the FS temperature is low and the Fermi edge is sharp [75. Therefore, one should expect delayed dephasing and thermalization processes as the electrons approach the Fermi surface (note that the above phase space restriction does no apply to the $e-h$ scattering of the photoexcited hole with the FS electrons).

The effect of a cold FS on the dephasing time of the interband optical polarization was investigated by Kim et al. [76] using transient FWM spectroscopy [9]. In such experiments, two optical pulses separated by a time delay $\tau$ propagate along two different directions, $\mathbf{k}_{\mathbf{1}}$ and $\mathbf{k}_{\mathbf{2}}$, and interfere within the sample, thus generating a nonlinear polarization. In FWM spectroscopy, one measures the signal that emerges along the direction $2 \mathbf{k}_{\mathbf{2}}-\mathbf{k}_{\mathbf{1}}$. In the case of optically-thin samples, the time-resolved FWM signal corresponds to the square of the amplitude of the nonlinear polarization along the above direction [9]. The time-integrated FWM signal (TI-FWM) is given by the integral over all times of the time-resolved FWM signal and is a function of the time delay $\tau$. To a first approximation, the FWM signal can be interpreted similar to atomic and molecular systems based on a two-level system [18, 9]. Within such a model, in the case of homogeneous broadening, the time-resolved FWM signal is emitted immediately after the second 
pulse arrives and decays with a decay time $T_{2} / 2$, where $T_{2}$ is the dephasing time. The TI-FWM signal also decays with a decay time $T_{2} / 2$ and vanishes for negative time delays where pulse 1 comes after pulse 2 . In the case of inhomogeneous broadening, the time-resolved FWM signal is delayed by a time interval $\tau$ after the second pulse arrives and corresponds to a photon echo [18, 9, 19]. The TI-FWM signal decays with a decay time $T_{2} / 4$, which is a factor of 2 smaller than in the case of homogeneous broadening, and also vanishes for negative time delays. In undoped semiconductors, significant deviations from the above simple picture have been observed and have been attributed to Hartree-Fock exciton-exciton interactions and to exciton-exciton correlations [12]. For example, such effects lead to a strong TI-FWM signal for negative time delays as well as a delayed time-resolved FWM signal. For a detailed review of the exciton-exciton interaction effects in undoped semiconductors, see e.g. Ref. [12.

In MDQW's, Kim et. al. measured long dephasing times of a few picoseconds for energies within the frequency range of the FES (i.e. close to $\left.E_{F}\right)$. These decreased to less than 100fs as the excitation frequency exceeded the FES peak by an energy comparable to the Fermi energy. The above result is consistent with the Fermi liquid energy dependence of the $e-e$ scattering time, Eq. 7, and points out that, unlike for the $e-h$ correlations, in MDQW's the $e-e$ scattering is suppressed within the frequency range of the FES. This experiment also suggests that the hole dephasing times are of the order of a few picoseconds. In the time-resolved FWM signal, Kim et. al. observed photon-echo-like behavior, which indicates that, despite the strong FES peak in the linear absorption, the continuum of interband $e-h$ states leads to behavior similar to that of an inhomogeneously-broadened system. Finally, in MDQW's, the pair-pair interactions, analogous to the excitonexciton interactions in undoped semiconductors, are screened out and the negative time delay TI-FWM signal characteristic of exciton-exciton interactions was absent for high Fermi sea densities [76]. The above experimental results were interpreted by Hawrylak et. al. [74 within the two-level system approximation (which neglects all FES correlation effects) by introducing energy-dependent dephasing times determined by the $e-e$ and $e-p$ scattering times.

In order to provide further insight into the dephasing processes in MDQW's, Bar-Ad et. al. [77 performed low temperature FWM measurements in the presence of a magnetic field parallel to the QW growth axis. For zero magnetic field, they observed a dephasing time $\sim 1$ ps. For magnetic fields such 
that only the lowest Landau level is occupied, they observed a suppression of the $e-e$ scattering. In particular, the dephasing times were then found to be longer, $\sim 1.5 \mathrm{ps}$, and, unlike in the zero field case of Ref. [76], they did not depend significantly on the frequency of the optical excitation. Bar Ad et. al. attributed the above observations to the suppression of the phase space available for scattering induced by the magnetic confinement. Indeed, the application of a magnetic field changes the continuous density of states into a series of discrete highly degenerate Landau level peaks, broadened by the disorder. Finally, Bar-Ad et. al. observed a FES in the FWM spectrum at high magnetic fields, which they attributed to the optical transitions to the occupied lowest Landau level.

The dephasing of the intersubband optical polarization in MDQW's was recently investigated by Kaindl et. al. [78 using transient FWM in the midinfrared. They observed an exponential decay of the TI-FWM signal that was fairly insensitive to the temperature. By modelling their data with a HFA calculation, Kaindl at. al. deduced the values of the dephasing times to be of the order of hundreds of femtoseconds and concluded that the main dephasing mechanism is the $e-e$ scattering. By increasing the Fermi sea density, they observed faster dephasing. In another experiment, Bonvalet et. al. [79] used an extremely short, $\sim 12 \mathrm{fs}$, pulse to excite a wavepacket consisting of states in the two lowest conduction subbands of a QW. By measuring the radiated coherent electromagnetic field, they observed a quantum beat at room temperature oscillating at the inverse intersubband excitation energy. From the decay of the signal they deduced an intersubband polarization dephasing time of 110fs in the case of a MDQW, which was shorter than the dephasing time of $180 \mathrm{fs}$ observed in an undoped QW. Such polarization dephasing is determined by the destructive interference effects as well as by the different scattering processes.

The above measurements of the polarization dephasing were performed under resonant photoexcitation conditions. In contrast to undoped semiconductors, the relaxation of such real pump-induced $e-h$ pairs due to their $e-e$ interactions with the FS will obscure any coherent effects. Fortunately, the dissipative processes can be suppressed by tuning the pump frequency below the onset of absorption. In this case, only virtual carriers are excited by the pump field and thus the coherent effects will dominate. For well-belowresonance pump excitation, the $e-h$ pair phase is primarily determined by the optical field and the time dependence of the interband polarization follows that of the pump pulse (adiabatic following [19]). In this case, the pump 
creates a truly coherent $e-h$ pair many-body state that lasts for the duration of the pulse. Such pump-probe experiments in atomic systems showed a resonance blueshift ( optical Stark effect) accompanied by a resonance bleaching due to the Phase Space Filling by the pump-induced $e-h$ pairs [19, 5, 80]. In the case of excitons, it was shown that the Coulomb interaction can significantly alter the bleaching observed in atomic systems. In particular, at low pump intensities or for pulse duration longer than the dephasing time, the interactions lead to a pure exciton blueshift without significant bleaching. For high intensities and pulse duration shorter than the dephasing time, the exciton bleaching is very strong. The exciton ac-Stark effect has been reviewed e.g. in Refs. [5, 6, 15, 12, 81]. Such interaction effects in undoped semiconductors suggest that the many-body $e-h$ correlations between the photoexcited holes and the FS electrons will significantly affect the coherent nonlinear optical response of the FES. Brener et. al. [82, 83 studied this issue by performing pump-probe measurements for pump detunings $\sim$ $50 \mathrm{meV}$ below the Fermi surface, much larger than the $e-h$ Coulomb energy $E_{M}$ (which is much smaller than the Fermi energy $\sim 15 \mathrm{meV}$ ). They observed a qualitatively different bleaching of the FES as compared to excitons in undoped QW's, which they attributed to the distinct nature of the two resonances. Schäfer [82] studied this issue by performing quantum kinetic calculations using Keldysh Green's functions. Such an approach treats the HFA coherent effects and includes the scattering contributions within the second order Born, Markovian, and static screening approximations. The numerical results for Fermi energy equal to the exciton binding energy showed a strikingly different bleaching between the FES and the exciton, which was attributed to the $e-e$ scattering.

As we discuss at length in section 5, the $e-h$ correlations between the photoexcited hole and the FS electrons lead to strong dephasing and affect qualitatively the coherent ultrafast dynamics of the FES. An important difference from undoped semiconductors is that, due to its gapless excitation spectrum, a FS responds unadiabatically to time-dependent perturbations. In contrast, because of its finite Coulomb binding energy, an exciton can be polarized by the pump optical field without being ionized. A non-equilibrium treatment beyond the HFA is necessary in order to take into account the unadiabatic time-dependent change in the $e-h$ pair-FS interactions and the $e-h$ scattering processes induced by the ultrafast pump excitation in the coherent regime. As has already been noted in the case of photoexcitation of an electron gas within the continuum of states of an undoped semiconductor 84, 
the loss of coherence due to the many-body correlations cannot be fully described within the dephasing time approximation. In section 5 we show that memory effects due to the $e-h$ correlations lead to a time evolution of the pump-probe signal characterized by the inverse Coulomb energy $E_{M}$ rather than by the dephasing time (as in the case of exciton bound states).

Concluding this section, let us briefly discuss a very recent FWM experiment by Fromer et. al. 85, which demonstrated for the first time nonMarkovian memory effects in the Quantum Hall Effect regime. Even though for magnetic fields between 5.5 and $6.5 \mathrm{~T}$ the TI-FWM profile was found to be a single exponential with an unusually long decay time, for magnetic fields that exceed $7.5 \mathrm{~T}$ such a profile was more complicated and characterized by a change of slope indicating memory effects in the polarization dynamics. Such effects were also seen in the frequency domain: the FWM spectrum profile changed from a Lorentzian lineshape to an asymmetric one corresponding to a frequency dependent width, $\Gamma(\omega)$. To interpret such behavior, we note that, at high magnetic fields such that the cyclotron energy, $\hbar \omega_{c}$, is large compared to other characteristic energies of the system, the relaxation is dominated by intra-Landau-level processes. Such scattering by collective excitations involves the matrix elements of the dynamically screened interaction, $U_{i j}^{<}\left(t, t^{\prime}\right)$, which in the lowest Landau level have the form:

$$
U_{i j}^{<}\left(t, t^{\prime}\right)=\int \frac{d \mathbf{q}}{(2 \pi)^{2}} e^{-q^{2} l^{2} / 2} v_{q}^{2} \bar{\chi}_{q}^{<}\left(t, t^{\prime}\right) c_{i j}(q),
$$

where $\bar{\chi}_{q}^{<}\left(t, t^{\prime}\right)=\left\langle\bar{\rho}_{\mathbf{q}}\left(t^{\prime}\right) \bar{\rho}_{-\mathbf{q}}(t)\right\rangle$ is the density-density correlation function projected onto the lowest Landau level [86, 87], and $\bar{\rho}_{\mathbf{q}}(t)$ is the corresponding density operator. Here, $v_{q}$ is the unscreened Coulomb interaction, $l=(\hbar / e B)^{1 / 2}$ is the magnetic length, and the coefficients $c_{i j}(q)$ with $i, j \rightarrow e, h$ model the asymmetry in the $e$ - $e$ and $e-h$ interaction matrix elements, which originates from the difference between the electron and hole lowest Landau level wavefunctions. Because of the breakdown of perturbation theory due to the Landau level degeneracy in 2D systems, it is incorrect to evaluate $\chi_{q}^{<}\left(t, t^{\prime}\right)$ within the standard RPA [10]. Instead, one should account for the true excitations of the interacting two-dimensional electron liquid. Several models can be found in the literature, and we base our discussion on the magnetoroton model, which is the one best suited for the filling factors $\nu$ in the experiment of Ref. [85]. The most salient features are, however, general and model independent. The magnetoroton dephasing mechanism is somewhat similar to that of acoustic phonon scattering. Under 
the experimental conditions of Ref. [85], to a very good approximation, the intra-Landau-level collective excitations are not affected by the small density of photogenerated carriers, so one can use the equilibrium density correlation function 86]. The equations for the density matrix elements then read

$$
\begin{aligned}
& \left.\frac{\partial \rho_{i j}}{\partial t}\right|_{s c a t t}=i \sum_{k} \int_{-\infty}^{t} d t^{\prime} G_{i}^{r}\left(t-t^{\prime}\right) G_{j}^{a}\left(t^{\prime}-t\right) \\
& \times\left(\left[U_{i k}^{<}\left(t-t^{\prime}\right)-U_{k j}^{<}\left(t-t^{\prime}\right)\right] \rho_{i k}^{<}\left(t^{\prime}\right) \rho_{k j}^{>}\left(t^{\prime}\right)-(<\leftrightarrow>)\right),
\end{aligned}
$$

where $G_{i}^{r / a}(t)$ is the retarded/advanced Green function, $\rho_{i j}^{<}=\rho_{i j}$, and $\rho_{i j}^{>}=$ $\delta_{i j}-\rho_{i j}$. If all $U_{i j}$ are equal, i.e., $c_{i j}(q)=1$, then the polarization scattering term vanishes [88]. This corresponds to identical electron and hole wavefunctions in the lowest Landau level. In practice, there is always an asymmetry between electrons and holes, due to, e.g., differing band offsets, lateral confinement, and disorder. Using the results of Ref. [86], Eq. (8) takes the form

$$
\begin{aligned}
U^{<}(t)=-\frac{i n}{2 \pi} \int \quad & \frac{d \mathbf{q}}{(2 \pi)^{2}} e^{-q^{2} l^{2} / 2} v_{q}^{2} c_{i j}(q) \\
& \times \bar{s}_{q}\left[\left(N_{q}+1\right) e^{i \omega_{q} t}+N_{q} e^{-i \omega_{q} t}\right],
\end{aligned}
$$

where $N_{q}$ is the Bose distribution function for magnetorotons of energy $\omega_{q}$, and $\bar{s}_{q}$ is the static stucture factor of the 2D electron liquid in the lowest Landau level. By comparing Eqs. (9) and (10), we see that the $\omega$ dependence of $\Gamma(\omega)$ is determined by the Fourier transform of $U^{<}(t)$, which is governed by the $q$ dependence of $\bar{s}_{q}$. In the lowest Landau level, we have $\bar{s}_{q}=\left(2 \nu^{-1}-\right.$ 1) $\tilde{s}_{q}$, where $\tilde{s}_{q} \sim(q l)^{4}$ for $q l \ll 1, \sim \exp \left(-q^{2} l^{2} / 2\right)$ for $q l \gg 1$, and $\tilde{s}_{q}$ displays a peak for $q l \sim 186$ that leads to the magnetoroton excitations. The corresponding resonance in $\Gamma(\omega)$ near the magnetoroton energy leads to nonMarkovian behavior with a characteristic response time of approximately the inverse of this energy. The latter is estimated from the gap $\Delta$ at the magnetoroton dispersion minimum, $\Delta \sim 0.1\left(e^{2} / \epsilon l\right)$ for the range of $\nu$ in this experiment [86], which for $B=10 \mathrm{~T}$ is $\approx 1.5 \mathrm{meV}$.

This concludes our overview of the main ultrafast dynamical features observed in MDQW's. In the next section we discuss the ultrafast dynamics in the case of metal nanoparticles and compare to MDQW's. 


\section{Nonlinear Optical Dynamics in Metal Nanopar- ticles}

The properties of small metal particles in the intermediate regime between bulk-like and molecular behavior have been the subject of great interest lately. This was motivated in part by the need to understand how the properties of matter evolve at the transition from atoms and molecules to bulk solids. An additional motivation comes from the technological trend towards electronic and optoelectronic devices based on smaller and smaller solid state structures [39, 90, 91, 92, 93]. Metal clusters are also being used in a variety of applications, ranging from catalysis to biological and medical applications ( see e.g. Refs. 994, 95, 96, 97, 98]). The electronic and thermodynamic properties of metal clusters have been extensively reviewed, e.g. in Refs. [99, 100, 101, 102]. The linear optical properties of metal nanoparticles have also been reviewed in detail by Kreibig and Vollmer [103].

It has been known for a long time that surface collective excitations play an important role in the absorption of light by metal nanoparticles. In large particles with sizes comparable to the wave-length of light $\lambda$ (but smaller than the bulk mean free path), the lineshape of the surface plasmon (SP) resonance is determined by the electromagnetic effects [104, 105, 106, 103]. However, as the size of the nanoparticle becomes smaller than the mean free path of electrons in the bulk metal, quantum confinement becomes important. In small nanoparticles with radii $R \ll \lambda$, the absorption spectrum is governed by quantum confinement effects. For example, the momentum nonconservation due to the confining potential leads to the Landau damping of the SP and to a resonance linewidth inversely proportional to the nanoparticle size [107]. A review of the extensive theoretical and experimental studies of this effect may be found in Ref. [103]. More recently, it was established that the static nonlinear optical properties of small nanoparticles are also affected by the confinement. In particular, a size-dependent enhancement of the third-order nonlinear optical susceptibilities for monochromatic photoexcitation, caused by the elastic surface scattering of single-particle excitations, was predicted by Flytzanis and collaborators [108, 109] and observed experimentally by Yang et. al. [110]. Dielectric confinement also enhances the optical nonlinearities close to the SP frequency [109, 103, [11]. Before we proceed with the discussion of the ultrafast dynamics of such confined Fermi seas, let us briefly summarize in the next section the main features of the SP 
resonance in the linear absorption spectrum.

\subsection{Surface Plasmon Resonance in Linear absorption}

In this section we summarize the basic facts regarding the linear absorption by small metal particles embedded in a medium with dielectric constant $\epsilon_{m}$. We will focus primarily on noble metal particles containing several hundreds of atoms; in this case, the confinement affects the extended electronic states even though the bulk lattice structure has been established. When the particle radii are small, $R \ll \lambda$, so that only dipole surface modes can be optically excited and non-local effects can be neglected, the optical properties of this system are determined by the dielectric function 103

$$
\epsilon_{\mathrm{col}}(\omega)=\epsilon_{m}+3 p \epsilon_{m} \frac{\epsilon(\omega)-\epsilon_{m}}{\epsilon(\omega)+2 \epsilon_{m}}
$$

where $\epsilon(\omega)=\epsilon^{\prime}(\omega)+i \epsilon^{\prime \prime}(\omega)$ is the dielectric function of a metal particle and $p \ll 1$ is the volume fraction occupied by nanoparticles in the colloid. Since the $d$-electrons play an important role in the optical properties of noble metals, the dielectric function $\epsilon(\omega)$ includes also the interband contribution $\epsilon_{d}(\omega)$ due to transitions from the $\mathrm{d}$-band to the s-p conduction band. For

$p \ll 1$, the absorption coefficient of such a system is proportional to that of a single particle and is given by 103

$$
\alpha(\omega)=-9 p \epsilon_{m}^{3 / 2} \frac{\omega}{c} \operatorname{Im} \frac{1}{\epsilon_{s}(\omega)},
$$

where

$$
\epsilon_{s}(\omega)=\epsilon_{d}(\omega)-\omega_{p}^{2} / \omega\left(\omega+i \gamma_{s}\right)+2 \epsilon_{m}
$$

plays the role of an effective dielectric function of a particle in the medium. Its zero, $\epsilon_{s}^{\prime}\left(\omega_{s}\right)=0$, determines the frequency of the SP, $\omega_{s}$. In Eq. (13), $\omega_{p}$ is the bulk plasmon frequency of the conduction electrons, and the width $\gamma_{s}$ characterizes the SP damping. The semiclassical result Eqs. (12) and (13) applies to nanoparticles with radii $R \gg q_{T F}^{-1}$, where $q_{T F}$ is the ThomasFermi screening wave-vector $\left(q_{T F}^{-1} \sim 1 \AA\right.$ in noble metals). In this case, the electron density deviates from its classical shape only within a surface layer occupying a small fraction of the total volume [112]. Quantum mechanical corrections, arising from the discrete energy spectrum, lead to a width $\gamma_{s} \sim v_{F} / R$, where $v_{F}=k_{F} / m$ is the Fermi velocity [103, 107]. Even though 
$\gamma_{s} / \omega_{s} \sim\left(q_{T F} R\right)^{-1} \ll 1$, this damping mechanism dominates for sizes $R<10$ $\mathrm{nm}$. Additional contributions to such a width come from the $e-p, e-e$, and electron-impurity interactions as well as from the disorder. On the other hand, in small clusters containing several dozens of atoms, this semiclassical approximation breaks down and density functional or ab initio methods should be used [103, 99, 100, 101, 102, 113. In particular, discrete electronic levels whose width does not exceed their spacing are expected for particle sizes smaller than a few angstroms. In the latter regime, the $e-e$ interactions are similar to those in atoms and molecules. One therefore expects that the role of the many-body correlations increases as we approach the crossover from quasi-continuous to discrete nanoparticle energy levels.

It should be noted that, in contrast to the scattering with surface collective excitations, the $e-e$ scattering is not too sensitive to the nanoparticle size as long as the condition $q_{T F} R \gg 1$ holds [114]. Indeed, for such sizes, the static screening is essentially bulk-like. At the same time, the energy dependence of the bulk $e-e$ scattering rate (Eq. 7) $\gamma_{e} \propto\left(E-E_{F}\right)^{2}$ comes from the phase-space restriction due to the energy and momentum conservation, and involves the exchange of typical momenta $q \sim q_{T F}$. If the size-induced momentum uncertainty $\delta q \sim R^{-1}$ is much smaller than $q_{T F}$, the $e-e$ scattering rate in a nanoparticle is not significantly affected by the confinement [115. Below we will see however that this is not the case for the electron-SP interactions.

\subsection{Ultrafast Dynamics}

Even though the electronic, thermodynamic, and optical properties of metal nanoparticles have been extensively studied, the role of confinement in the electron dynamics is much less understood. Examples of outstanding issues include the role of $e-e$ interactions in the process of cluster fragmentation, the role of surface lattice modes in providing additional channels for intramolecular energy relaxation, the influence of the electron and nuclear motion on the superparamagnetic properties of clusters, and the effect of confinement on the nonlinear optical properties and transient response under ultrafast excitation [103, 113]. These and other time-dependent phenomena can be studied with femtosecond nonlinear optical spectroscopy, which in these structures provides time resolution shorter than the relaxation times. Similar experimental studies in bulk metals and metal interfaces and surfaces have been reviewed e.g. in Ref. [17]. Here we will focus on the more recent work 
in metal nanoparticles.

Extensive experimental studies of the electron relaxation in noble-metal nanoparticles have recently been performed using ultrafast pump-probe spectroscopy. In contrast to the situation in semiconductors, the dephasing processes in metals are very fast. In metal nanoparticles, the dephasing time can be deduced, e.g., from the linear absorption SP width to be of the order of a few fs. Such measurements were performed by Klar et. al. [116, who distinguished between homogeneous and inhomogeneous broadening by measuring directly the SP lineshape of a single nanoparticle using a near-field antenna effect. The decay time of the SP resonance has also been studied with second and third harmonic generation measurements [117, 118].

Within several femtoseconds after the photoexcitation, the opticallyexcited electrons and holes form non-equilibrium populations. The pumpinduced intraband transitions create a broad non-thermal electron distribution that extends from the Fermi energy up to the pump photon energy, while the interband transitions create additional electron and d-band hole populations when the pump frequency exceeds the $\mathrm{d}$-band to conduction band transition threshold. Within a few fs, the high energy electrons scatter to lower energies due to $e-e$ interactions with the FS electrons. The latter interactions are screened within time intervals of the order of the inverse plasma frequency, typically $\sim 1$ fs. Note that, similar to MDQW's [74], at high electron excess energies the $e-e$ scattering time is of the order of a fs, much shorter than the $e-p$ scattering times (of the order of a ps). However, as such electrons scatter down to the Fermi surface, the $e-e$ scattering times become much longer, of the order of several hundreds of fs. As discussed in the previous section, this is a consequence of the Pauli blocking of the phase space available for scattering and the screening of the $e-e$ interaction, which leads to energy-dependent scattering times given by Eq. 7 [1]. Therefore, one expects that thermalization should slow down as the electrons approach the Fermi surface. In the case of metal films, a non-thermal population consisting of a hot Fermi-Dirac distribution together with a tail of high energy electrons right above the Fermi surface (and a tail of low energy holes below the Fermi surface) was indeed observed in time-resolved photoemission experiments during time intervals of the order of hundreds of fs [119, 120]. Pump-probe experiments also showed a clear signature of delayed thermalization (see e.g. [121, 122, 123, 124, 125]). In particular, the rise time of the differential transmission and reflectance signal was observed to be of the order of hundreds of fs, much longer than the pulse duration. 
A similar slow rise time of the differential transmission was also observed in the case of copper [126], gold [127], and silver [128] nanoparticles as well as in gold nanoshells [129, 130]. It has been reported that, in small nanoparticles with diameters $\sim 5 \mathrm{~nm}$, such a delayed thermalization regime (stage $\mathbf{I I}$ ) lasts longer than in metal films 126, 128. At the same time, the $e-p$ interaction for such sizes was found to be weaker as compared to the bulk [126, 128]. A possible explanation of the reduced $e-p$ interaction is that, for the small sizes, the characteristic phonon energy, given by the Debye frequency, becomes smaller than the spacing between the nanoparticle energy levels close to the Fermi surface, which is of the order of $v_{F} / R$. As a result, the scattering of an electron via bulk-like phonons is suppressed [131. As the electrons approach the Fermi surface, the $e-e$ scattering times become comparable to the $e-p$ scattering times and therefore the $e-p$ interaction also contributes to the thermalization of the electron gas. The suppression of the latter could lead to delayed internal thermalization of the electron gas.

The main spectral feature in the differential absorption of the copper and gold nanoparticles (where the onset of interband transitions is very close to SP frequency) was a transient asymmetric broadening of the SP resonance ( see e.g. Refs. [132, 133, 134, 135, 136, 126, 127, 137, 138]). Perner et. al 127 observed a build-up of such a pump-induced broadening during time intervals of the order of 1ps. Bigot et. al. [126 observed a similar buildup and also pointed out that the differential absorption lineshape could not be understood without including the effects of the energy-dependent $e^{-}$ $e$ scattering on the interband dielectric function. They attributed the SP broadening mainly to the smearing of the Fermi-Dirac electron distribution close to the Fermi surface, due to the heating of the electron gas, which affects the interband dielectric function. Inouye et.al. 137 arrived at similar conclusions and argued that, in the case of resonant interband excitations, the contribution of the intraband $e-e$ scattering to the damping of the SP, which is described by the width $\gamma_{s}$ in the intraband dielectric function in Eq. (13) 125, 139, 140, plays a minor role.

The time-delay of the SP broadening can be understood as follows. For quasi-equilibrium conditions, the nonlinear absorption spectrum can be described using the linear absorption results but with dielectric constants determined by the time-dependent carrier populations. As discussed above, within the time resolution of the experiment, the electron distribution deviates from the equilibrium Fermi-Dirac distribution in the vicinity of the Fermi surface, due to the high electron temperature and the non-thermal 
population [119, 120, 121, 122]. As can be seen from the linear absorption expressions, such a smearing of the FS distribution leads to the broadening of the SP resonance. Therefore, the initial rise time of the differential transmission signal is determined by the non-equilibrium electrons ouside the equilibrium Fermi-Dirac distribution. Initially, such electrons occupy the broad photoexcited distribution and therefore their number is relatively small, determined by the pump intensity. However, their number increases with time as the $e-e$ interaction leads to the scattering of more electrons out of the FS. This is the origin of the time-dependent increase in the SP broadening.

Since the electron heat capacity is much smaller than that of the lattice, an electron temperature much higher than that of the lattice can be reached during subpicosecond time scales. Subsequently, the electron and phonon baths equilibrate through the $e-p$ interactions over time intervals of a few picoseconds. During this incoherent stage, the hot electrons can be characterized by a thermalized Fermi-Dirac distribution with time-dependent temperature $T(t)$, while the phonons can be characterized by a Bose-Einstein distribution with time-dependent temperature $T_{l}(t)$. During the cooling of the hot electron gas, the SP width decreases 1132, 133, 134, 135, 136, 126, 127, 137, 138. As soon as the electrons have equilibrated among themselves, one can study the subsequent time evolution by using a set of coupled differential equations for the temperatures $T(t)$ and $T_{l}(t)$, referred to as the two-temperature model [141, 142, 144, 145, 146, 147]:

$$
\begin{aligned}
C_{e}(T) \frac{\partial T}{\partial t} & =\nabla \cdot\left(\kappa_{e} \nabla T\right)-G\left(T-T_{l}\right)+P(\mathbf{r}, t), \\
C_{l} \frac{\partial T_{l}}{\partial t} & =G\left(T-T_{l}\right),
\end{aligned}
$$

where $C_{e}(T)=\gamma T$ and $C_{l}$ are the electron and lattice heat capacities, respectively, $G$ is the energy transfer coefficient between the electrons and the lattice, which is proportional to the $e-p$ coupling constant [143], $\kappa_{e}$ is the electronic thermal conductivity, and the source term $P(\mathbf{r}, t)$ describes the local energy density per unit time absorbed by the electron system from the pump optical pulse. The first term on the rhs of Eq. (14) describes the thermal diffusion from the nanoparticle to the surrounding matrix, which occurs on a time scale of several tens of ps. The second term on the rhs of Eq. (14) determines the tranfer of heat from the electron gas to the lattice as the former cools down during a time interval of several ps. The above model as- 
sumes electron and lattice temperatures larger than the Debye temperature 1142 and that the interactions can maintain a quasi-equilibrium for both the electron and the phonon populations at all times. The cooling of the electron gas to the lattice manifests itself via an exponential decay of the differential transmission. According to Eq. (14), the decay time of the differential tranmission signal is inversely proportional to the strength of the $e-p$ interaction [123, 124]. Stella et. al. [148] performed transient reflectivity measurements in metallic tin nanoparticles with radii ranging from $2 \mathrm{~nm}$ to $6 \mathrm{~nm}$ and saw a decay of the signal as a function of pump-probe delay that became faster by decreasing the nanoparticle radius. They deduced from their data a contribution to the energy relaxation time during the thermalization of the hot electron Fermi sea with the lattice that was inversely proportional to the nanoparticle radius. In a subsequent paper, Nisoli et. al. [149 performed femtosecond pump-probe measurements in solid and liquid gallium nanoparticles with radii ranging from $5 \mathrm{~nm}$ to $9 \mathrm{~nm}$ and observed energy relaxation time constants that varied from 1.6 ps to 600 fs with decreasing nanoparticle size. They observed similar electron relaxation dynamics in the solid and liquid nanoparticle phases, which indicates that in this system, the scattering of electrons with bulk phonons plays only a minor role in the relaxation because of the reduction in the available phase space due to the quantum confinement. Instead, the hot electrons transfer their excess energy to the lattice through the generation of surface vibrational waves (capillary waves), which leads to an electron-surface phonon interaction $G$ inversely proportional to the nanoparticle radius [150]. An additional contribution to this effect may come from the fact that the heat diffusion to the matrix becomes faster with decreasing nanoparticle size due to a thermal buildup that occurs for larger particles. A similar effect was observed by Halte et. al. in the case of very small silver nanoparticles [151]. The role of surface effects on the dynamics was also discussed in Ref. 136.

In the gold and copper nanoparticles, condidered above, the SP frequency is very close to the onset of interband transitions. In contrast, in silver nanoparticles, the SP and interband transitions are well separated in energy. Halte et. al. 128 compared the electron dynamics between silver thin films and silver nanoparticles embedded in glass in the same spectral range, and studied its dependence on the photoexcitation intensity. In thin films, they found that the RPA dielectric function corresponding to the instantaneous temperature determined by the two-temperature model reproduced their results quite well. In the case of the silver nanoparticles, the main feature 
in the differential absorption spectrum was an apparent SP redshift, which was attributed to the changes in the real part of the interband dielectric function due to the thermal broadening of the electron distribution close to the Fermi level. It was also shown that the finite $e-e$ lifetime of the carriers photoexcited via interband transitions was essential for obtaining such a redshift. Finally, the strong non-parabolicity of the conduction band at the energies corresponding to the optical frequencies was shown to be important for interpreting the data. Averitt et. al. [129, 130] performed ultrafast pump-probe measurements in gold nanoshell structures, which consist of a dielectric core surrounded by a thin metallic shell of nanometer dimensions. In such structures, the SP frequency can be tuned by changing the ratio of the core diameter to the shell thickness, and the experiment was performed at frequencies far off the onset of the interband transitions. The delayed pumpinduced broadening of the SP resonance suggests that a nonthermal electron population is present in these structures during the initial $\sim 100$ fs following the pump photoexcitation. The decay of the pump-probe signal was found to be somewhat slower than in the bulk, which was attributed to a weaker $e-p$ interaction due to the reduction of phase space available for scattering with bulk phonons induced by the confinement. Finally, they observed a transient blueshift of the SP resonance, attributed to the off-resonant interband transitions.

Recent experimental results in the case of small noble metal particles [126, 128] indicate that many-body correlation effects play an important role during the cooling of the electron gas to the lattice (relaxation stage III). Despite the similarities to the bulk-like behavior, observed, e.g., in metal films, certain aspects of the optical dynamics in nanoparticles are significantly different 126, 152, 153. For example, the experimental studies of copper nanoparticles by Bigot et. al. [126] revealed that, for sizes smaller than $5 \mathrm{~nm}$, the decay times of the pump-probe signal depend strongly on the probe frequency in the immediate vicinity of the SP resonance. In particular, the relaxation is considerably slower at the SP resonance, and becomes faster right above and right below the SP frequency [126, 152, 153]. This and other observations suggest that collective surface excitations play an important role in the electron dynamics in small metal particles. This important issue will be discussed at length in section 7 . 


\section{Coherent Ultrafast Response of the Fermi Edge Singularity: Formalism}

\subsection{Basic equations}

Before we proceed with our discussion of the time evolution of the FES in the coherent regime, we outline the main points of a formalism [154, 155, 83, 156, 157 recently developed to account for the non-Markovian dynamics due to the dynamical FS response ( $e-h$ correlations). More details may be found in Appendix A.

As discussed in section 2.1, even in linear absorption, the HFA and the dephasing time approximation have serious shortcomings when used to describe the FES. In the case of a photoexcited electron gas, the correlation effects have been treated within the second Born approximation by using Keldysh Green functions (see e.g. Ref. [10]). However, at low temperatures and within the frequency range of the FES, the effects of the $e-h$ interaction must be consistently accounted for to arbitrary order. For example, as discussed in section 2.1, even in linear absorption the vertex correction diagrams with arbitrarily many crossed $e-h$ interaction lines are as divergent as the HFA ladder diagrams and should therefore be treated on equal footing. With Green functions, this requires summing up at least the parquet diagrams 42, 47, a formidable task especially in the non-equilibrium femtosecond regime. Alternative methods are therefore desirable. In undoped semiconductors, the "dynamic controlled truncation scheme" has been used to treat the exciton-exciton correlations [11, 26] (see section 1). However, such a hierarchy of density matrix equations no longer truncates if the ground state of the semiconductor includes a FS. Furthermore, as we demonstrate in section 5.1, an expansion in terms of the optical fields breaks down for frequencies within the FES range. Finally, in view of the significant complexity of the problem, it is highly desirable to use a method that also allows for a physically intuitive interpretation of the results. The purpose of this section is to outline the main points of such a method, which will be used in section 5 to describe the coherent nonlinear response of the FES.

We first consider the case of one conduction subband and later extend the formalism to include the Coulomb coupling to a second subband. We also consider spinless electrons for simplicity. In the rotating frame [19, the 
Hamiltonian describing this system is

$$
H_{\mathrm{tot}}(t)=H+H_{p}(t)+H_{s}(t) .
$$

The first term is the "bare" Hamiltonian,

$$
H=\sum_{\mathbf{q}} \varepsilon_{\mathbf{q}}^{v} b_{-\mathbf{q}}^{\dagger} b_{-\mathbf{q}}+\sum_{\mathbf{q}}\left(\varepsilon_{\mathbf{q}}^{c}+\Omega\right) a_{\mathbf{q}}^{\dagger} a_{\mathbf{q}}+V_{e e}+V_{h h}+V_{e h},
$$

where $a_{\mathbf{q}}^{\dagger}$ is the creation operator of a conduction electron with energy $\varepsilon_{\mathbf{q}}^{c}$ and mass $m_{e}, b_{-\mathbf{q}}^{\dagger}$ is the creation operator of a valence hole with energy $\varepsilon_{\mathbf{q}}^{v}$ and mass $m_{h}, V_{e e}, V_{e h}$, and $V_{h h}$ describe the $e-e, e-h$, and $h-h$ interactions, respectively, and $\Omega=E_{g}+E_{F}\left(1+m_{e} / m_{h}\right)-\omega_{p}$ is the detuning of the central frequency of the optical fields, $\omega_{p}$, from the Fermi level, $E_{g}$ being the bandgap (we set $\hbar=1$ everywhere). The second and third terms describe the coupling of the pump optical field, $\mathcal{E}_{p}(t) e^{i \mathbf{k}_{p} \cdot \mathbf{r}-i \omega_{p} t}$, and the probe optical field, $\mathcal{E}_{s}(t) e^{i \mathbf{k}_{s} \cdot \mathbf{r}-i \omega_{p}(t-\tau)}$, respectively:

$$
\begin{gathered}
H_{p}(t)=-\mu \mathcal{E}_{p}(t)\left[e^{i \mathbf{k}_{p} \cdot \mathbf{r}} U^{\dagger}+\text { H.c. }\right], \\
H_{s}(t)=-\mu \mathcal{E}_{s}(t)\left[e^{i \mathbf{k}_{s} \cdot \mathbf{r}+i \omega_{p} \tau} U^{\dagger}+\text { H.c. }\right],
\end{gathered}
$$

where the pump amplitude $\mathcal{E}_{p}(t)$ is centered at time $t=0$ and the probe amplitude $\mathcal{E}_{s}(t)$ is centered at the time delay $t=\tau, \mu$ is the interband transition matrix element, and

$$
U^{\dagger}=\sum_{\mathbf{q}} a_{\mathbf{q}}^{\dagger} b_{-\mathbf{q}}^{\dagger}
$$

is the optical transition operator. The conventions for the time delay $\tau$ are clarified in Appendix B.

In many experiments, the amplitude of the probe field is much smaller than that of the pump, $\left|\mathcal{E}_{p}(t)\right| \gg\left|\mathcal{E}_{s}(t)\right|$. In that case, as was shown in Ref. [154] (see Appendix A), the experimentally-measurable nonlinear optical polarization can be obtained in terms of the linear response functions of a "pump-dressed" semiconductor to a probe field (note that, within $\chi^{(3)}$, this is true even for comparable pulse amplitudes). This "dressed" system is described by a time-dependent effective Hamiltonian $\tilde{H}(t)$, which is obtained by performing a time-dependent Schrieffer-Wolff/Van Vleck canonical transformation on the Hamiltonian $H+H_{p}(t)$ [158, 159, 160, 161]. As we shall 
see later, in all the cases of interest, the effective Hamiltonian $\tilde{H}(t)$ has the same operator form as the bare Hamiltonian $H$, with the important difference that the band dispersions (effective masses) and interaction potentials are time-dependent through $\mathcal{E}_{p}(t)$. Thus, the calculation of the nonlinear absorption spectrum reduces to that of the linear absorption spectrum of the "pump-dressed" semiconductor with uncoupled "effective bands" — a great simplification that allows us to use straightforward generalizations of well established theoretical tools in order to treat the correlations. In fact, such an approach mimics nicely the spirit of the pump-probe experiments and allows for a physically intuitive interpetation of the results. It is important to note here that this "pump-dressed semiconductor" approach is not restricted to monochromatic pulses and is valid for any pulse duration [154].

The pump-probe nonlinear polarization has the following form (see Appendix A):

$$
P_{\mathbf{k}_{s}}(t)=i \mu^{2} e^{i \mathbf{k}_{s} \cdot \mathbf{r}-i \omega_{p}(t-\tau)} \int_{-\infty}^{t} d t^{\prime} \mathcal{E}_{s}\left(t^{\prime}\right)\left\langle\Phi_{0}(t)\left|\tilde{U}(t) \mathcal{K}\left(t, t^{\prime}\right) \tilde{U}^{\dagger}\left(t^{\prime}\right)\right| \Phi_{0}\left(t^{\prime}\right)\right\rangle .
$$

Here, $\left|\Phi_{0}(t)\right\rangle$ is the state evolved with $\tilde{H}(t)$ from the semiconductor ground state $|0\rangle$ of $H, \tilde{U}^{\dagger}(t)$ is the effective optical transition operator describing the probability amplitude for the photoexcitation of an $e-h$ pair by the probe field in the presence of the pump excitation, and $\mathcal{K}\left(t, t^{\prime}\right)$ is the time-evolution operator satisfying the Schrödinger equation

$$
i \frac{\partial}{\partial t} \mathcal{K}\left(t, t^{\prime}\right)=\tilde{H}(t) \mathcal{K}\left(t, t^{\prime}\right)
$$

The above equation describes the time evolution of a probe-photoexcited $e-h$ pair in the presence of the pump excitation. The effective Hamiltonian and effective transition operator are given by (see Appendix A)

$$
\tilde{H}(t)=H_{0}+\frac{\mu}{2}\left(\mathcal{E}_{p}(t)\left[\hat{\mathcal{P}}(t), U^{\dagger}\right]+\text { H.c. }\right),
$$

and

$$
\tilde{U}^{\dagger}(t)=U^{\dagger}+\frac{1}{2}\left[\hat{\mathcal{P}}(t),\left[U^{\dagger}, \hat{\mathcal{P}}^{\dagger}(t)\right]\right]+\frac{1}{2}\left[\hat{\mathcal{P}}^{\dagger}(t),\left[U^{\dagger}, \hat{\mathcal{P}}(t)\right]\right],
$$

where the operator $\hat{\mathcal{P}}^{\dagger}(t)$, which generates the canonical transformation, satisfies the equation

$$
i \frac{\partial \hat{\mathcal{P}}^{\dagger}(t)}{\partial t}=\left[H, \hat{\mathcal{P}}^{\dagger}(t)\right]+\mu \mathcal{E}_{p}(t) U^{\dagger}
$$


with the initial condition $\hat{\mathcal{P}}^{\dagger}=0$ before the pump arrives. Eqs. (21) and (22) include all the pump-induced corrections to $\tilde{U}^{\dagger}(t)$ and $\tilde{H}(t)$ up to the second order in the pump optical field and are valid when $\left(\mu E_{p} / \Omega\right)^{2}<1$ (for off-resonant excitation) or $\left(\mu E_{p} t_{p}\right)^{2}<1$ (for resonant excitation), where $t_{p}$ is the pump duration.

It should be emphasized that, although Eq. (21) gives the effective Hamiltonian up to the second order in the pump field, $\mathcal{E}_{p} \mathcal{E}_{p}^{*}$, the polarization expression Eq. (19) describes the effects of $\tilde{H}$ in all orders. For example, as we shall see in section 4.3 , the pump-induced term in $\tilde{H}$ contains self-energy corrections to the electron/hole energies, which describe (among other effects) the resonance blueshift due to the ac-Stark effect. Although the magnitude of these self-energy corrections, calculated using Eq. (21), is quadratic in $\mathcal{E}_{p}$, the correct position of the resonance can only be obtained by evaluating the PP polarization (19) nonperturbatively (beyond $\chi^{(3)}$ ), i.e., without resorting to the expansion of the time-evolution operator $\mathcal{K}\left(t, t^{\prime}\right)$ in the pump field. Importantly, as we demonstrate in 5.1, the same is true when calculating the effects of the self-energy corrections on the $e-h$ correlations. As we shall see in section 5, such a nonperturbative (in the pump field) treatment of the nonlinear response of the FES is crucial for the adequate description of the PP spectrum at negative time delays. In Sections 4.2 and 4.4, we will describe the corresponding procedure, which accounts for the FS dynamical response. In contrast, the third-order polarization, $\chi^{(3)}$, can be simply obtained from Eq. (19) by expanding $\mathcal{K}\left(t, t^{\prime}\right)$ to the first order in the pump-induced term in $\tilde{H}$ [second term in Eq. (21)]. We did not include in Eq. (19) the biexcitonic contribution (coming from the excitation of two $e-h$ pairs by the pump and the probe pulses) since it vanishes for the negative time delays $(\tau<0)$ considered below where the coherent effects dominate [154].

In addition to including important contributions beyond $\chi^{(3)}$ via the solution of Eq. (20) as discussed above, the advantage of Eq. (19), as compared to the equations of motion for the polarization, comes from its similarity to the linear polarization that determines the linear absorption spectrum [43]. This can be seen by setting $\mathcal{E}_{p}(t)=0$ in the Eqs. (21) and (22), in which case the effective time-evolution and optical transition operators transform into their "bare" counterparts: $\mathcal{K}\left(t, t^{\prime}\right) \rightarrow e^{-i H\left(t-t^{\prime}\right)}$ and $\tilde{U}^{\dagger}(t) \rightarrow U^{\dagger}$. Moreover, like $U^{\dagger}$, the effective transition operator $\tilde{U}^{\dagger}(t)$ creates a single $e-h$ pair, while, as we shall see in section 4.3, the effective Hamiltonian $\tilde{H}(t)$ can be cast in a form similar to $H$. This allows one to interpret the Fourier transform of 
Eq. (19) as the linear absorption spectrum of a "pump-dressed" semiconductor with two uncoupled but time-dependent effective bands. This mapping simplifies significantly the calculation of the FES ultrafast nonlinear optical response by allowing a straightforward generalization of the $\mathrm{CCE}$. It also allows one to interpret the various dynamical features in the nonlinear absorption spectra, originating from the correlation effects, within the familiar framework developed for linear spectroscopy.

\subsection{Overview of the Coupled Cluster Expansion}

In this section, we show how the time-dependent CCE can be used to study the effects of the $e-h$ correlations (dynamical FS response) on the time evolution of the $e-h$ pair photoexcited by the probe. Our goal is to evaluate the many-body state $|\Psi(t)\rangle=\mathcal{K}\left(t, t^{\prime}\right) \tilde{U}^{\dagger}\left(t^{\prime}\right)\left|\Phi_{0}\left(t^{\prime}\right)\right\rangle$ that enters in Eq. (19). This state satisfies the Schrödinger equation

$$
i \frac{\partial}{\partial t}|\Psi(t)\rangle=\tilde{H}(t)|\Psi(t)\rangle
$$

As already mentioned, $\tilde{H}(t)$ has the same form as the bare Hamiltonian $H$. This allows us to obtain $|\Psi(t)\rangle$ through a straightforward generalization of the linear absorption calculations [55, 56, 57, 58]. After eliminating the valence hole degrees of freedom [162, 58], $|\Psi(t)\rangle$ is expressed in the CCE form 52

$$
|\Psi(t)\rangle=e^{S(t)}|\Phi(t)\rangle,
$$

where the time-dependent operator $S(t)$ creates FS-pairs and is given by

$$
\begin{aligned}
S(t)= & \sum_{p>k_{F}, k<k_{F}} s(\mathbf{p}, \mathbf{k}, t) a_{\mathbf{p}}^{\dagger} a_{\mathbf{k}} \\
& +\sum_{p_{1}, p_{2}>k_{F}, k_{1}, k_{2}<k_{F}} s_{2}\left(\mathbf{p}_{\mathbf{1}}, \mathbf{p}_{\mathbf{2}}, \mathbf{k}_{\mathbf{1}}, \mathbf{k}_{\mathbf{2}}, t\right) a_{\mathbf{p}_{\mathbf{1}}}^{\dagger} a_{\mathbf{p}_{\mathbf{2}}}^{\dagger} a_{\mathbf{k}_{\mathbf{2}}} a_{\mathbf{k}_{\mathbf{1}}}+\cdots
\end{aligned}
$$

while the state $|\Phi(t)\rangle$, discussed in Section 4.4, describes the time evolution of the probe-induced $e-h$ pair. In Eq. (26), the amplitude $s(\mathbf{p}, \mathbf{k}, t)$ describes

the $e-h$ correlations which, in particular, are responsible for the unbinding of the HFA bound state; the two-pair amplitude $s_{2}$ describes the $e-e$ interaction effects at the RPA level and beyond. ¿From a physical point of view, the operator $e^{S(t)}$ describes the readjustment of the FS density profile during the 
optical transition in response to the FS interactions with the photoexcited $e-h$ pair.

Substituting Eq. (25) into the Schrödinger equation Eq. (24), multiplying by the operator $e^{-S(t)}$ from the lhs, and using the fact that $\left[S(t), S\left(t^{\prime}\right)\right]=$ $\left[\dot{S}(t), S\left(t^{\prime}\right)\right]=0$, one obtains

$$
i \frac{\partial}{\partial t}|\Phi(t)\rangle+i \dot{S}(t)|\Phi(t)\rangle=e^{-S(t)} \tilde{H}(t) e^{S(t)}|\Phi(t)\rangle,
$$

where the transformed Hamiltonian on the rhs can be expressed in terms of the commutator series (Baker-Campbell-Hausdorff expansion)

$$
e^{-S(t)} \tilde{H}(t) e^{S(t)}=\tilde{H}(t)+[\tilde{H}(t), S(t)]+\frac{1}{2}[[H, S(t)], S(t)]+\cdots
$$

An important advantage of the CCE is that, due to the FS momentum restrictions in Eq. (26), the above series terminates after the first few terms (three for quartic interactions) and a closed-form expression of the transformed Hamiltonian (28) can be obtained in terms of $S(t)$. By requiring that all FS-pair creation processes are eliminated from the above equation, one obtains the CCE equations [52, 55] for $S(t)$. Before proceeding with such

a calculation however, one needs to derive explicit expressions for $\tilde{H}(t)$ and $\tilde{U}(t)$.

\subsection{Effective Hamiltonian and Transition Matrix Ele- ments}

\subsubsection{Discussion for Doped Semiconductors}

In the general case, the effective Hamiltonian $\tilde{H}(t)$ is given by Eq. (21) and the effective optical transition operator $\tilde{U}(t)$ by Eq. (22). For calculations, it is useful to re-express such equations in second-quantized form. In the case of undoped semiconductors, it was shown in Ref. [154] that the effective Hamiltonian $\tilde{H}(t)$ describes the same interactions as the bare Hamiltonian $H$, however among quasiparticles with time-dependent properties determined by the pump polarization. Similarly, the effective interband transition matrix element in $\tilde{U}(t)$ was shown to have an imaginary part that describes the dephasing due to the exciton-exciton interactions. The purpose of this section is to present similar results in the case of a doped semiconductor 157, 156, 83. 
We start with Eqs. (21) and (22), which express $\tilde{H}(t)$ and $\tilde{U}(t)$ in terms of the canonical transformation operator $\hat{\mathcal{P}}^{\dagger}(t)$. The latter is given by Eq. (23), which includes the effects of the Coulomb interactions on the pump photoexcitation. It is important to realize that the effect of the $e-h$ interactions on the pump and the probe photoexcitations is very different. For an adequate description of the FES, the $e-h$ interactions should be taken into account non-perturbatively for the probe-photoexcited pair. Indeed, the nonlinear absorption spectrum at a given frequency $\omega$ close to the FES resonance is determined by the time-evolution for long times (of the order of the dephasing time $T_{2}$ ) of an $e-h$ pair photoexcited by the probe at the energy $\omega$ [163. Since the characteristic " $e-h$ interaction time" $E_{M}^{-1}$ (inverse HFA bound state energy) that determines the non-exponential polarization decay of the FES is much shorter that the dephasing time, the long-time asymptotics of the response function (to the probe) depends non-perturbatively on the $e-h$ interactions. In contrast, a short pump optical pulse excites a wavepacket of continuum $e-h$ pair states (unlike in the discrete exciton case) with energy width $\sim t_{p}^{-1}$, which thus evolves during timescales comparable to the pulse duration $t_{p}$. Also, the corrections to the effective Hamiltonian are determined by the time evolution of the pump-induced carriers only up to times $\sim t_{p}$ [see Eq. (21)]. Therefore, if the " $e-h$ interaction time" $E_{M}^{-1}$ is larger than $t_{p}, t_{p} E_{M}<1$, (i.e., if the pump pulse frequency width exceeds $\left.E_{M}\right)$, the $e-h$ interactions can be treated perturbatively when describing the time-evolution of the pump-photoexcited pairs. This can also be shown explicitly for the third-order nonlinear polarization. In the general expression for $\chi^{(3)}$, all contributions that depend on the pump are integrated over the width of the pump pulse; therefore, any resonant enhancement of $\chi^{(3)}$ that depends on the pump frequency will be broadened out for sufficiently short pulses with frequency width that exceeds $E_{M}$. In other words, when deriving the pump-renormalized parameters, one can treat Coulomb interactions perturbatively if the above condition is fulfilled. In fact, the above situation is somewhat similar to the calculation of the linear absorption spectrum close to the indirect transition threshold, where perturbation theory can be used 42, 47. Thus the above consideration applies even for long pulse durations provided that the detuning $\Omega$ exceeds $E_{M}$. However, in order to obtain the full absorption spectrum, the time-evolution of the probe-photoexcited pair with such effective Hamiltonian (with perturbatively calculated timedependent parameters) should be treated non-perturbatively. As can be seen from the above discussion, an important advantage of this formalism is that 
it seprates naturally between the perturbative and the non-perturbative interaction effects.

\subsubsection{Second Quantization Expressions}

We now proceed with the derivation of the effective Hamiltonian. To lowest order in the interactions, $\hat{\mathcal{P}}^{\dagger}(t)$ can be presented as

$$
\begin{aligned}
\hat{\mathcal{P}}^{\dagger}(t)= & \sum_{\mathbf{q}} \mathcal{P}_{e h}(\mathbf{q}, t) a_{\mathbf{q}}^{\dagger} b_{-\mathbf{q}}^{\dagger}+\frac{1}{2} \sum_{\mathbf{p}, \mathbf{p}^{\prime}, \mathbf{k}} \mathcal{P}_{e h}^{e}\left(\mathbf{p} \mathbf{p}^{\prime} ; \mathbf{k} ; t\right) b_{\mathbf{k}-\mathbf{p}-\mathbf{p}^{\prime}}^{\dagger} a_{\mathbf{p}^{\dagger}}^{\dagger} a_{\mathbf{p}^{\prime}}^{\dagger} a_{\mathbf{k}} \\
& +\frac{1}{2} \sum_{\mathbf{p}, \mathbf{p}^{\prime}, \mathbf{k}} \mathcal{P}_{e h}^{h}\left(\mathbf{p} \mathbf{p}^{\prime} ; \mathbf{k} ; t\right) a_{\mathbf{p}+\mathbf{p}^{\prime}-\mathbf{k}}^{\dagger} b_{-\mathbf{p}}^{\dagger} b_{-\mathbf{p}^{\prime}}^{\dagger} b_{-\mathbf{k}},
\end{aligned}
$$

where $\mathcal{P}_{e h}$ is the probability amplitude for excitation of an $\mathrm{e}-\mathrm{h}$ pair with zero momentum satisfying

$$
i \frac{\partial}{\partial t} \mathcal{P}_{e h}(\mathbf{q}, t)=\left[\Omega+\varepsilon_{\mathbf{q}}^{(c)}+\varepsilon_{-\mathbf{q}}^{(v)}\right] \mathcal{P}_{e h}(\mathbf{q}, t)+\mu \mathcal{E}_{p}(t)-\sum_{\mathbf{q}^{\prime}} v\left(\mathbf{q}-\mathbf{q}^{\prime}\right) \mathcal{P}_{e h}\left(\mathbf{q}^{\prime}, t\right)
$$

In Eq. (29),

$$
\begin{aligned}
\mathcal{P}_{e h}^{e}\left(\mathbf{p p}^{\prime} ; \mathbf{k} ; t\right) & =i \int_{-\infty}^{t} d t^{\prime} e^{-i\left(t-t^{\prime}\right)\left(\Omega+\varepsilon_{\mathbf{p}}^{c}+\varepsilon_{\mathbf{p}^{\prime}}^{c}-\varepsilon_{\mathbf{k}}^{c}+\varepsilon_{\mathbf{p}+\mathbf{p}^{\prime}-\mathbf{k}}^{v}\right)} \\
\times & {\left[v(\mathbf{p}-\mathbf{k})\left[\mathcal{P}_{e h}\left(\mathbf{p}^{\prime}, t^{\prime}\right)-\mathcal{P}_{e h}\left(\mathbf{p}+\mathbf{p}^{\prime}-\mathbf{k}, t^{\prime}\right)\right]-\left(\mathbf{p} \leftrightarrow \mathbf{p}^{\prime}\right)\right] }
\end{aligned}
$$

describes the scattering of the photoexcited $e-h$ pair with an electron, and

$$
\begin{aligned}
\mathcal{P}_{e h}^{h}\left(\mathbf{p p}^{\prime} ; \mathbf{k} ; t\right) & =-i \int_{-\infty}^{t} d t^{\prime} e^{-i\left(t-t^{\prime}\right)\left(\Omega+\varepsilon_{\mathbf{p}+\mathbf{p}^{\prime}-\mathbf{k}}^{c}+\varepsilon_{\mathbf{p}^{\prime}}^{v}+\varepsilon_{\mathbf{p}}^{v}-\varepsilon_{\mathbf{k}}^{v}\right)} \\
\times & {\left[v(\mathbf{p}-\mathbf{k})\left[\mathcal{P}_{e h}\left(\mathbf{p}^{\prime}, t^{\prime}\right)-\mathcal{P}_{e h}\left(\mathbf{p}+\mathbf{p}^{\prime}-\mathbf{k}, t^{\prime}\right)\right]-\left(\mathbf{p} \leftrightarrow \mathbf{p}^{\prime}\right)\right] }
\end{aligned}
$$

describes the scattering of the photoexcited $e-h$ pair with a hole. The above expressions describe in the lowest order in the screened interaction [164] $v(\mathbf{p}-\mathbf{k})$ the coherent pump-induced processes, the effects of the HartreeFock pair-pair and pair-FS interactions, and the dynamical FS response to the pump photoexcitation.

By substituting Eq. (29) into Eq. (22), we obtain the following expression for the effective optical transition operator:

$$
\begin{aligned}
\tilde{U}^{\dagger}(t)\left|\Phi_{0}(t)\right\rangle= & \sum_{p>k_{F}} M_{\mathbf{p}}(t) a_{\mathbf{p}}^{\dagger} b_{-\mathbf{p}}^{\dagger}|0\rangle \\
& +\frac{1}{4} \sum_{p, p^{\prime}>k_{F}, k<k_{F}} M_{\mathbf{p p}^{\prime} \mathbf{k}}(t) a_{\mathbf{p}}^{\dagger} a_{\mathbf{p}^{\prime}}^{\dagger} b_{\mathbf{k}-\mathbf{p}-\mathbf{p}^{\prime}}^{\dagger} a_{\mathbf{k}}|0\rangle,
\end{aligned}
$$


where the effective matrix element $M_{\mathbf{p}}(t)$ includes corrections due to phase space filling and Hartree-Fock interactions, and $M_{\mathbf{p p}^{\prime} \mathbf{k}}(t)$ is the probability amplitude for indirect optical transitions [42] induced by the pump optical field, which contribute to the pump-probe polarization in the second order in the interactions. The explicit expressions for $M_{\mathbf{p}}(t)$ and $M_{\mathbf{p p}^{\prime} \mathbf{k}}(t)$ are given in Appendix C.

We turn now to the effective Hamiltonian $\tilde{H}(t)$. After substituting Eq. (29) into Eq. (21) we obtain that

$$
\tilde{H}(t)=\sum_{\mathbf{q}} \varepsilon_{\mathbf{q}}^{v}(t) b_{-\mathbf{q}}^{\dagger} b_{-\mathbf{q}}+\sum_{\mathbf{q}} \varepsilon_{\mathbf{q}}^{c}(t) a_{\mathbf{q}}^{\dagger} a_{\mathbf{q}}+V_{e h}(t)+V_{e e}(t),
$$

where

$$
\varepsilon_{\mathbf{q}}^{c}(t)=\varepsilon_{\mathbf{q}}^{c}-\mu \mathcal{E}_{p}(t) \operatorname{Re}\left[\mathcal{P}_{e h}(\mathbf{q}, t)-\sum_{\mathbf{q}^{\prime}} \mathcal{P}_{e h}^{e}\left(\mathbf{q} \mathbf{q}^{\prime} ; \mathbf{q} ; t\right)\right]
$$

is the effective conduction electron energy;

$$
\varepsilon_{\mathbf{q}}^{v}(t)=E_{g}+\varepsilon_{\mathbf{q}}^{v}-\mu \mathcal{E}_{p}(t) \operatorname{Re}\left[\mathcal{P}_{e h}(\mathbf{q}, t)-\sum_{\mathbf{q}^{\prime}} \mathcal{P}_{e h}^{h}\left(\mathbf{q q}^{\prime} ; \mathbf{q} ; t\right)\right],
$$

is the effective valence hole energy;

$$
V_{e h}(t)=-\sum_{\mathbf{k k}^{\prime} \mathbf{q}} v_{e h}\left(\mathbf{q} ; \mathbf{k} \mathbf{k}^{\prime} ; t\right) a_{\mathbf{k}+\mathbf{q}}^{\dagger} a_{\mathbf{k}} b_{-\mathbf{k}^{\prime}-\mathbf{q}}^{\dagger} b_{-\mathbf{k}^{\prime}}
$$

is the effective $e-h$ interaction; and

$$
V_{e e}(t)=\frac{1}{2} \sum_{\mathbf{k k}^{\prime} \mathbf{q}} v_{e e}\left(\mathbf{q} ; \mathbf{k k}^{\prime} ; t\right) a_{\mathbf{k}+\mathbf{q}}^{\dagger} a_{\mathbf{k}^{\prime}-\mathbf{q}}^{\dagger} a_{\mathbf{k}^{\prime}} a_{\mathbf{k}}
$$

is the effective $e-e$ interaction. The explicit expressions for $v_{e h}\left(\mathbf{q} ; \mathbf{k k}^{\prime} ; t\right)$ and $v_{e e}\left(\mathbf{q} ; \mathbf{k k}^{\prime} ; t\right)$ are given in Appendix C. As can be seen, $\tilde{H}(t)$ has the same operator form as the bare Hamiltonian $H$. However, both the effective band dispersions and the effective interaction potentials are now dependent on time. Note here that the above pump-induced renormalizations only last for the pulse duration $t_{p}$. As discussed above, they are therefore perturbative in the screened interactions for $t_{p} E_{M}<1$ or for $\Omega>E_{M}$.

Let us first discuss the effect of the pump-induced self-energy corrections to the conduction and valence band energies, given by the last terms in 
Eqs. (35) and (36). The dispersion of the effective band is shown in Fig. 1. As can be seen, the pump pulse leads to a bandgap increase as well as a change in the momentum dependence (band dispersion) that last as long as the pump pulse. The magnitude of the bandgap increase is of the order of $\left(\mu \mathcal{E}_{p}\right)^{2} / \Omega$ (for off-resonant excitation) and $\left(\mu \mathcal{E}_{p}\right)^{2} t_{p}$ (for resonant excitation) and leads to, e.g., the ac-Stark blueshift. As we shall see, for pulse duration shorter than the dephasing time, it also leads to bleaching and gain right below the onset of absorption, analogous to the case of excitons or twolevel systems. It should be emphasized that these are coherent effects that should not be confused with the incoherent bandgap redshift due to the $e-e$ interactions among real photoexcited carriers [63]. In particular, the above bandgap renormalization is induced by the transverse EM-field of the laser, as compared to the usual bandgap renormalization due to a longitudinal EMfield, i.e., Coulomb screening. The change in the band dispersion, whose relative magnitude is of the order of $\left(\mu \mathcal{E}_{p} / \Omega\right)^{2}$ (for off-resonant excitation) or $\left(\mu \mathcal{E}_{p} t_{p}\right)^{2}$ (for resonant excitation), can be viewed as an increase in the effective density of states and, to the first approximation, in the effective mass. This is important in doped semiconductors because, as we shall see later, it leads to an optically-induced time-dependent enhancement of the $e-h$ interactions and scattering processes with the FS electrons.

The effective Hamiltonian $\tilde{H}(t)$ also includes pump-induced corrections in the effective interaction potentials, determined by the pair-pair and pairFS interactions during the pump photoexcitation. By expanding Eqs. (128) and (129) for carrier energies close to the Fermi surface using Eqs. (31) and (32), one can show that these corrections vanish at the Fermi surface; for the typical FS excitation energies $\Delta \varepsilon \sim E_{M}$ that contribute to the FES, their order of magnitude is $\left(\mu \mathcal{E}_{p} \Delta \varepsilon / \Omega^{2}\right)^{2}$ (for off-resonant excitation) or $\left(\mu \mathcal{E}_{p} \Delta \varepsilon t_{p}^{2}\right)^{2}$ (for resonant excitation). Thus the corrections to the interaction potentials are suppressed for below-resonant excitation by a factor of $\left(E_{M} / \Omega\right)^{2}$, or for short pulses by a factor of $\left(E_{M} t_{p}\right)^{2}$, as compared to the self-energy corrections. Such a suppression is due to the Pauli blocking effect and the screening, which leads to the vanishing of the pump-induced corrections to the interaction potentials at the Fermi surface. Similarly, the pump-induced indirect optical transition matrix elements $M_{\mathbf{p p}^{\prime} \mathbf{k}}(t)$ are suppressed by the same factor as compared to the direct transition matrix element $M_{\mathbf{p}}(t)$ [first term in Eq. (33)], while they contribute to the pump-probe polarization only in the second order in the screened interactions. Therefore, in the doped case, the screened Coulomb interaction leads to subdominant parameter renormal- 
izations to the effective Hamiltonian $\tilde{H}(t)$ and transition operator $\tilde{U}^{\dagger}(t)$ for sufficiently short pump pulses or for off-resonant excitation. In contrast, in undoped semiconductors, the exciton-exciton interactions lead to nonperturbative contributions discussed in Ref. [154.

\subsection{Description of the Electron-Hole Pair Dynamics}

In this section, we present the final formulae for the nonlinear pump-probe polarization of the interacting system by applying the CCE to the effective Hamiltonian $\tilde{H}(t)$ in order to treat the dynamical FS response. The CCE equation Eq. (27) contains the operator $S(t)$ described by a hierarchy of coupled equations for the amplitudes $s, s_{2}, \cdots$, defined by Eq. (26). As discussed at length above, in the coherent regime of interest here, the $e-e$ scattering effects are suppressed, and the nonlinear absorption spectrum is dominated by the $e-h$ interactions. This allows us to use the dephasing time approximation for treating the probe-induced $e-e$ scattering processes [48, [50, 51], in which case the above hierarchy terminates after $s$. Importantly, the $e-h$ correlations (dynamical FS response) are still treated non-perturbatively, since they are determined by $s$ [55, 56, 58]. Then all FS-pair creation processes can be eliminated explicitely from the rhs of Eq. (27), leading to the following nonlinear differential equation for the one-FS-pair scattering amplitude [55, 56, 58, $s(\mathbf{p}, \mathbf{k}, t)$ :

$$
\begin{aligned}
i \frac{\partial s(\mathbf{p}, \mathbf{k}, t)}{\partial t}- & {\left[\varepsilon_{\mathbf{p}}^{c}(t)-\varepsilon_{\mathbf{k}}^{c}(t)\right] s(\mathbf{p}, \mathbf{k}, t)=} \\
& V\left[1+\sum_{p^{\prime}>k_{F}} s\left(\mathbf{p}^{\prime}, \mathbf{k}, t\right)\right]\left[1-\sum_{k^{\prime}<k_{F}} s\left(\mathbf{p}, \mathbf{k}^{\prime}, t\right)\right] .
\end{aligned}
$$

The $e-h$ scattering processes described by the above equation are sketched in Fig. 2a. Here $V$ is the s-wave component [5, 49] of the screened interaction [164, approximated for simplicity by its value at the Fermi energy [42, 50, 48]. This neglects plasmon effects, which are however small within the frequency range of the FES [165, 74]. Although this approximation is standard for the linear absorption case, its justification for the transient spectra requires more attention. Indeed, the characteristic time for screening buildup is of the order $\Omega_{p}^{-1}$, where $\Omega_{p}$ is the typical plasma frequency corresponding to the FS [166, 167, 163. This time is however shorter than the typical pump duration $\sim 100 \mathrm{fs}$ and dephasing time (which is of the order of ps near the Fermi 
energy [76]), so the Coulomb interactions can be considered screened also for the nonlinear absorption case. In Eq. (39), we neglected the hole recoil energy contribution to the excitation energy [58] since $\varepsilon_{k_{F}}^{v}(t)-\varepsilon_{0}^{v}(t)<E_{M}$ due to a sufficiently heavy hole mass [42]. Note that, by increasing the hole effective mass, the pump-induced hole self-energy, Eq. (36), reduces the hole recoil energy and thus the corresponding broadening 42. In real samples, the relaxation of the momentum conservation condition due to the disorder will also suppress the hole recoil broadening effects. In fact, the disorder can even lead to localized hole states [35], which corresponds to the infinite hole mass limit.

From Eq. (27) we then easily obtain the following expression for $|\Phi(t)\rangle$ :

$$
|\Phi(t)\rangle=\sum_{p>k_{F}} \Phi_{\mathbf{p}}\left(t, t^{\prime}\right) a_{\mathbf{p}}^{\dagger} b^{\dagger}|0\rangle
$$

where $b^{\dagger}$ is the creation operator of the zero-momentum hole state and the $e^{-}$ $h$ pair wavefunction $\Phi_{\mathbf{p}}\left(t ; t^{\prime}\right)$ satisfies the "Wannier-like" equation of motion,

$$
\begin{array}{r}
i \frac{\partial \Phi_{\mathbf{p}}\left(t, t^{\prime}\right)}{\partial t}=\left[\Omega+\varepsilon_{\mathbf{p}}^{c}(t)+\varepsilon_{-\mathbf{p}}^{v}(t)\right. \\
\left.-\epsilon_{A}(t)-i \Gamma_{\mathbf{p}}\right] \Phi_{\mathbf{p}}\left(t, t^{\prime}\right) \\
-\tilde{V}(\mathbf{p}, t) \sum_{p^{\prime}>k_{F}} \Phi_{\mathbf{p}^{\prime}}\left(t, t^{\prime}\right),
\end{array}
$$

where

$$
\tilde{V}(\mathbf{p}, t)=V\left[1-\sum_{k^{\prime}<k_{F}} s\left(\mathbf{p}, \mathbf{k}^{\prime}, t\right)\right],
$$

is the effective $e^{-} h$ potential whose time- and momentum-dependence is determined by the response of the FS electrons to their interactions with the probe-induced $e-h$ pair (vertex corrections) [sketched schematically in Fig. 2(b), responsible for the unbinding of the HFA bound state],

$$
\epsilon_{A}(t)=V \sum_{k^{\prime}<k_{F}}\left[1+\sum_{p^{\prime}>k_{F}} s\left(\mathbf{p}^{\prime}, \mathbf{k}^{\prime}, t\right)\right]
$$

is the self-energy due the to the sudden appearance of the photoexcited hole, which leads to non-exponential polarization decay [described by $\operatorname{Im} \epsilon_{A}(t)$ ] due to the Anderson orthogonality catastrophe [34] and a dynamical resonance redshift [described by $\operatorname{Re} \epsilon_{A}(t)$ ], and the dephasing width $\Gamma_{\mathbf{p}}$ describes all additional dephasing processes (due to $e-e$ interactions, hole recoil, and phonons). Eq. (41) should be solved with the initial condition 
$\Phi_{\mathbf{p}}\left(t^{\prime}, t^{\prime}\right)=M_{\mathbf{p}}\left(t^{\prime}\right)$, where the effective matrix element $M_{\mathbf{p}}\left(t^{\prime}\right)$ is defined by Eq. (33).

It is worth stressing here the analogy between Eqs. (40) and (41) and the corresponding problem in undoped semiconductors. Indeed, Eq. (40) is the direct analog of an exciton state, whereas again Eq. (41) is very similar to a Wannier equation. However, Eqs. (39) and (41) include the effects of the interactions between the probe-photoexcited $e-h$ pair and the FSexcitations, and the wavefunction $\Phi_{\mathbf{p}}\left(t, t^{\prime}\right)$ describes the propagation of the photoexcited pair "dressed" by the FS excitations. Such a "dressing" is due to the dynamical FS response, which leads to the dynamical screening of the effective $e-h$ interaction Eq. (42). The time-dependence of the latter is determined by the FS scattering amplitude $s(\mathbf{p}, \mathbf{k}, t)$ and is affected by the pump excitation as described by Eq. (39). One can easily verify that by setting $s(\mathbf{p}, \mathbf{k}, t)=0$ in Eq. (41), we recover the results of the Hartree-Fock (ladder diagram, static FS [42]) approximation. If one neglects the nonlinear (quadratic) term in Eq. (39), one recovers the three-body (Fadeev) equations [42, 168]. Note that the coupled equations for $\Phi_{\mathbf{p}}\left(t, t^{\prime}\right)$ and $s(\mathbf{p}, \mathbf{k}, t)$, obtained by neglecting the multipair excitations in Eq. (26), can be extended to include the hole recoil-induced corrections [58].

Using Eqs. (25), (26), and (40), we now can express the pump-probe polarization Eq. (19) in terms of the $e-h$ wavefunction $\Phi$ and the effective transition matrix element $M_{\mathrm{p}}$. Assuming, for simplicity, a delta-function probe pulse centered at time delay $\tau, \mathcal{E}_{s}(t)=\mathcal{E}_{s} \delta(t-\tau)$, we obtain a simple final expression:

$$
P_{\mathbf{k}_{s}}(t)=i \theta(t-\tau) \mu^{2} \mathcal{E}_{s} e^{i \mathbf{k}_{s} \cdot \mathbf{r}-i \omega_{p}(t-\tau)} \sum_{p>k_{F}} M_{\mathbf{p}}(t) \Phi_{\mathbf{p}}(t, \tau) .
$$

Eq. (44) expresses the pump-probe polarization in terms of two physically distinct contributions. First is the effective transition matrix element $M_{\mathbf{p}}(t)$, which includes the effects of pair-pair and pair-FS interactions and Phase space filling effects due to the pump-induced carriers present during the probe photoexcitation. Second is the wavefunction $\Phi_{\mathbf{p}}(t)$ of the $e-h$ pair photoexcited by the probe, whose time dependence, determined by Eqs. (41) and (39), describes the formation of the absorption resonance. Despite the formal similarities, there are two important differences between the doped and the undoped cases. First, in the doped case, the time evolution of the $e-h$ wavefunction $\Phi_{\mathbf{p}}(t)$ is strongly affected by the interplay between the $e-h$ correlations and the pump-induced transient changes in the bandgap 
and band dispersion relations. As we shall see later, this can be viewed as an excitation-induced dephasing. Second, unlike in the undoped case [154, the pump-induced corrections in the effective matrix element $M_{\mathbf{p}}(t)$ are perturbative in the screened interactions if the pump detuning or the pump frequency width exceed the Coulomb energy $E_{M}$.

\subsection{The Case of Two Coupled Subbands}

In this section, the above results are extended to the multisubband case in order to investigate the ultrafast PP dynamics of the FES-exciton hybrid formed in asymmetric QW's with partially occupied subbands 169, 170, 37. 171, 172, 173, 174]. In such one-sided MDQW structures, interband optical transitions from the valence band to several conduction subbands are allowed due to the finite overlap between the hole and electron envelope wave-functions. When the exciton Fano resonance from a higher empty conduction subband is nearly resonant (within a few meV) to the Fermi level, the FES coming from the lowest occupied subband is enhanced by over two orders of magnitude [37, 169, 170]. Such many-body effects on the linear spectrum have been described by using the simple two-subband Hamiltonian 171, 172, 173

$$
H=\sum_{i \mathbf{k}} \epsilon_{i \mathbf{k}}^{c} a_{i \mathbf{k}}^{\dagger} a_{i \mathbf{k}}+\sum_{\mathbf{k}}\left(\epsilon_{\mathbf{k}}^{v}+E_{g}\right) b_{-\mathbf{k}}^{\dagger} b_{-\mathbf{k}}-\sum_{i j} \sum_{\mathbf{p k q}} v_{i j}(\mathbf{q}) a_{i \mathbf{p}+\mathbf{q}}^{\dagger} a_{j \mathbf{p}} b_{\mathbf{k}-\mathbf{q}}^{\dagger} b_{\mathbf{k}}
$$

where $a_{i \mathbf{k}}^{\dagger}$ and $\epsilon_{i \mathbf{k}}^{c}$ are the creation operator and the energy of a conduction electron in the $i$ th subband, $b_{-\mathbf{k}}^{\dagger}$ and $\epsilon_{\mathbf{k}}^{v}$ are those of a valence hole $\left(E_{g}\right.$ is the bandgap), and $v_{i j}(\mathbf{q})$ is the screened $e-h$ interaction matrix with diagonal (off-diagonal) elements describing the intrasubband (intersubband) scattering. Due to the screening, the interaction potential is short-ranged and can be replaced by its s-wave component [5, 49]; close to the Fermi surface, $v_{i j}(\mathbf{q}) \simeq v_{i j}$ 171, 172, 43. Here we consider the case where only the first subband is occupied, but the Fermi level is close to the exciton level (with binding energy $E_{B}$ ) below the bottom of the second subband. For large values of the FES-exciton splitting $\Delta-E_{F}-E_{B}$, where $\Delta$ is the subband separation, the linear absorption spectrum consists of two well separated peaks, the lower corresponding to the FES from subband 1, and the higher corresponding to the Fano resonance from the exciton of subband 2 broadened by its coupling to the continuum of states in subband 1 . With decreasing $\Delta-E_{F}-E_{B}$, 
the FES and the exciton become hybridized due to the intersubband scattering arising from the Coulomb interaction. This results in the transfer of oscillator strength from the exciton to the FES and a strong enhancement of the absorption peak near the Fermi level due to the resonant scattering of the photoexcited electron by the exciton level [169, 170, 37, 171, 172, 173. Since the PP signal is linear in the probe field, the essential physics can be captured by assuming a $\delta$-function probe pulse, $\mathcal{E}_{\tau}(t)=\mathcal{E}_{\tau} e^{i \omega_{p} \tau} \delta(t-\tau)$, and a Gaussian pump pulse. The PP polarization has the form $(t>\tau)$

$$
P(t)=i \mathcal{E}_{s} e^{-i \omega_{p} t} \sum_{i j} \mu_{i} \mu_{j}\left\langle 0\left|\tilde{U}_{i}(t) \mathcal{K}(t, \tau) \tilde{U}_{j}^{\dagger}(\tau)\right| 0\right\rangle,
$$

where $\mathcal{K}(t, \tau)$ is the time-evolution operator for the effective Hamiltonian,

$$
\tilde{H}(t)=\sum_{i j \mathbf{k}} \epsilon_{i j \mathbf{k}}^{c}(t) a_{i \mathbf{k}}^{\dagger} a_{j \mathbf{k}}+\sum_{\mathbf{k}} \epsilon_{\mathbf{k}}^{v}(t) b_{-\mathbf{k}}^{\dagger} b_{-\mathbf{k}}+V_{e h}(t)+V_{e e}(t),
$$

where $V_{e h}$ and $V_{e e}$ are the effective $e-h$ and $e$ - $e$ interactions and $\tilde{U}_{i}^{\dagger}(t)$ is the effective transition operator given below. Here

$$
\epsilon_{i j \mathbf{k}}^{c}(t)=\delta_{i j} \epsilon_{i \mathbf{k}}^{c}+\Delta \epsilon_{i j \mathbf{k}}^{c}(t), \quad \epsilon_{\mathbf{k}}^{v}(t)=\epsilon_{\mathbf{k}}^{v}+\Omega+\Delta \epsilon_{\mathbf{k}}^{v}(t)
$$

are the band dispersions with pump-induced self-energies (to lowest order in the interactions)

$$
\begin{aligned}
\Delta \epsilon_{i j \mathbf{k}}^{c}(t) & =-\mathcal{E}_{p}(t)\left[\mu_{i} p_{j \mathbf{k}}^{*}(t)+\mu_{j} p_{i \mathbf{k}}(t)\right] / 2, \\
\Delta \epsilon_{\mathbf{k}}^{v}(t) & =-\mathcal{E}_{p}(t) \operatorname{Re} \sum_{i} \mu_{i} p_{i \mathbf{k}}(t),
\end{aligned}
$$

with $p_{i \mathbf{k}}(t)$ satisfying

$$
i \frac{\partial p_{i \mathbf{k}}(t)}{\partial t}=\left(\epsilon_{i \mathbf{k}}^{c}+\epsilon_{\mathbf{k}}^{v}+\Omega\right) p_{i \mathbf{k}}(t)-\sum_{j \mathbf{q}} v_{i j} p_{j \mathbf{q}}(t)+\mu_{i} \mathcal{E}(t)
$$

Note that the pump induces additional intersubband scattering, described by $\Delta \epsilon_{12 \mathbf{k}}^{c}(t)$. To lowest order in the interactions, the effective transition operator appearing in Eq. (46) is given by

$$
\tilde{U}_{i}^{\dagger}(t)=\sum_{j \mathbf{k}} \phi_{i j \mathbf{k}}(t) a_{j \mathbf{k}}^{\dagger} b_{-\mathbf{k}}^{\dagger},
$$


with

$$
\phi_{i j \mathbf{k}}(t)=\delta_{i j}\left[1-\frac{1}{2} \sum_{l}\left|p_{l \mathbf{k}}(t)\right|^{2}\right]-\frac{1}{2} p_{i \mathbf{k}}(t) p_{j \mathbf{k}}^{*}(t) .
$$

In the one-subband case and in the coherent limit, Eq. (52) takes a familiar form, $\phi_{\mathbf{k}}(t)=1-\left|p_{\mathbf{k}}(t)\right|^{2}$ — the usual Pauli blocking factor [157; in a multi-subband case, the latter is a matrix.

Similar to the one-subband case, the state $\mathcal{K}(t, \tau) \tilde{U}_{i}^{\dagger}(\tau)|0\rangle$, entering into (46), can be viewed as describing the propagation of the $e-h$ pair (with wavefunction $\left.\Phi_{i j}(\mathbf{k}, t)\right)$ excited by the probe pulse at time $\tau$, dressed by the scattering of the FS excitations (dynamical FS response). The latter leads to a dynamical broadening described by the amplitude $s_{i j}(\mathbf{p}, \mathbf{k}, t)$ that satisfies the differential equation [55, 157]

$$
\begin{aligned}
i \frac{\partial s_{i j}(\mathbf{p}, \mathbf{k}, t)}{\partial t}= & \left(\epsilon_{i \mathbf{p}}^{c}-\epsilon_{j \mathbf{k}}^{c}\right) s_{i j}(\mathbf{p}, \mathbf{k}, t) \\
& +\sum_{l}\left[\Delta \epsilon_{i \mathbf{p}}^{c}(t) s_{l j}(\mathbf{p}, \mathbf{k}, t)-\Delta \epsilon_{l j \mathbf{k}}^{c}(t) s_{i l}(\mathbf{p}, \mathbf{k}, t)\right] \\
& -\sum_{l} \tilde{v}_{i l}(\mathbf{p}, t)\left[\delta_{l j}+\sum_{q>k_{F}} s_{l j}(\mathbf{q}, \mathbf{k}, t)\right]
\end{aligned}
$$

with initial condition $s_{i j}(\mathbf{p}, \mathbf{k}, \tau)=0$, and $\mathbf{p}$ and $\mathbf{k}$ labeling respectively the (ith subband) FS electron and the ( $j$ th subband) FS hole. Since only the first subband is occupied, the only non-zero components of $s_{i j}$ are $s_{11}(\mathbf{p}, \mathbf{k}, t)$ and $s_{21}(\mathbf{p}, \mathbf{k}, t)$, which describe the intra and intersubband FS excitations respectively. The photoexcited $e-h$ pair wavefunction $\Phi_{i j}(\mathbf{k}, t, \tau)$ satisfies the Wannier-like equation

$$
\begin{aligned}
i \frac{\partial \Phi_{i j}(\mathbf{k}, t)}{\partial t}= & \sum_{l}\left[\epsilon_{i l \mathbf{k}}^{c}(t)+\delta_{l j}\left[\epsilon_{\mathbf{k}}^{v}(t)+\epsilon_{A}(t)\right]-i \Gamma\right] \Phi_{l j}(\mathbf{k}, t) \\
& -\sum_{l, q>k_{F}} \tilde{v}_{i l}(\mathbf{k}, t) \Phi_{l j}(\mathbf{q}, t)
\end{aligned}
$$

with initial condition $\Phi_{i j}(\mathbf{k}, \tau)=\phi_{i j \mathbf{k}}(\tau)$, where

$$
\epsilon_{A}(t)=-\sum_{k^{\prime}<k_{F}}\left[v_{11}+\sum_{p^{\prime}>k_{F}} s_{11}\left(\mathbf{p}^{\prime}, \mathbf{k}^{\prime}, t\right) v_{11}\right]
$$

is the self-energy due to the readjustment of the FS to the photoexcitation of a hole [157, 55] and $\Gamma$ is the inverse dephasing time due to all the processes not included in $H$. In Eqs. (53) and (54), 


$$
\tilde{v}_{i j}(\mathbf{k}, t)=v_{i j}-\sum_{l, k^{\prime}<k_{F}} s_{i l}\left(\mathbf{k}, \mathbf{k}^{\prime}, t\right) v_{l j}
$$

is the effective $e-h$ potential whose time-dependence is due to the dynamical FS response [157. Note that it is the interplay between this effective potential and the pump-induced self-energies that gives rise to the unadiabatic FS response to the pump field. In terms of $\Phi_{i j}(\mathbf{k}, t)$, the polarization (46) takes the simple form $(t>\tau)$

$$
P(t)=-i \mathcal{E}_{s} e^{-i \omega_{p} t} \sum_{i j l} \mu_{i} \mu_{j} \sum_{k>k_{F}} \Phi_{i l}(\mathbf{k}, t) \phi_{i j \mathbf{k}}^{*}(t),
$$

with $\phi_{i j}(\mathbf{k}, t)$ given by (52). The nonlinear absorption spectrum is then proportional to $\operatorname{Im} P(\omega)$, where $P(\omega)$ is the Fourier transform of the rhs of (57).

\section{Coherent Ultrafast Dynamics of the Fermi Edge Singularity}

\subsection{Discussion of the Physics: Monochromatic Excita- tion}

In this section, the role of the $e-h$ correlations in the nonlinear optical response of the FES is illustrated in a simple way for monochromatic excitation [156, 83, 155, 157. In the latter case, the theory discussed in the previous section applies for pump detunings larger than the characteristic Coulomb energy, $\Omega>E_{M}$. As discussed above, close to the Fermi edge, the linear absorption spectrum of the FES can be approximately described using the simple power law expression in Eq. (4). The monochromatic pump excitation leads to a resonance blueshift, originating from the shift in the effective band energies [see Fig. [1], and to a bleaching mainly due to the Pauli blocking (Phase Space Filling) which reduces the effective transition matrix element (analogous to the dressed atom picture [160]). More importantly, however, the pump-induced change in the band dispersion increases the density of states $\mathcal{N}$ close to the Fermi surface and thus also increases both the $e-h$ scattering strength $g=V \mathcal{N}$ and the phaseshift $\delta \sim \tan ^{-1}(\pi g)$ that determine the FES lineshape (see Eq. (丑). This, in turn, leads to an increase in the 
FES exponent $\beta$ that determines the resonance width. In contrast, in the case of a bound excitonic state of dimensionality $D$ and Bohr radius $a_{B}$, the resonance width remains unchanged, while the oscillator strength, $\propto a_{B}^{-D}$, increases by a factor $\sim(1+D \Delta m)$, where $\Delta m$ is the pump-induced change in the effective mass 155, 156, 83. Such an optically-induced enhancement of the exciton strength competes with the bleaching due to the Pauli blocking and the exciton-exciton interactions. This results in an almost rigid exciton blueshift, consistent with experiment [175, 176] and previous theoretical results [177, 178].

However, in the case of a FES resonance, the pump-induced change in the exponent $\delta$ leads to a stronger oscillator strength enhancement than for a bound exciton state. Obviously, such an enhancement cannot be described perturbatively, i.e., with an expansion in terms of the optical field, since, as can be seen from Eq. (4), the corresponding corrections to the absorption spectrum diverge logarithmically for frequencies $\omega$ at the Fermi edge. Eq. (4) shows that the effect of the pump on the FES can be thought of as an excitation-induced dephasing that affects the frequency dependence of the resonance; again, this is in contrast to the case of the exciton. In the time domain, this also implies a memory structure related to the response-time of the FS-excitations. Therefore, the qualitative differences between the nonlinear optical response of the FES and the exciton originate from the fact that an exciton is a discrete bound state, while the FES is a continuum many-body resonance. The FS responds unadiabatically to the pump-induced change in the density of states via an increase in the $e-h$ scattering of low-energy pair excitations. Such scattering processes, which determine the response of the Fermi sea to the hole potential in the course of the optical excitation, are responsible for the unbinding and broadening of the HFA bound state 163. Therefore, the pump field changes the broadening and dephasing effects even for below-resonant photoexcitation. On the other hand, due to the finite Coulomb binding energy of the exciton, the pump optical field can polarize such a bound state and change its Bohr radius without ionizing it.

\subsection{Results for Short-pulse Excitation}

In this section, we discuss the nonlinear absorption of the FES in the case of short pulse excitation. The results presented here were obtained by solving numerically the differential equations (41) and (39), using the Runge-Kutta method, for Gaussian pulses with duration $t_{p}=2.0 E_{F}^{-1}$ [157. In order 
to suppress the incoherent effects due to the $e-e$ scattering of real carrier populations with the FS, below-resonant pump excitation and negative time delays were considered. Under such excitation conditions, the coherent effects in which we are interested dominate, and the Coulomb-induced corrections to the effective parameters, discussed in section 4.3, are perturbative. The goal of this section is to study the role of the dynamical FS response $(e-h$ correlations) on the pump-probe dynamics. For this reason, we compare the results of the theory outlined in section 1 to those of the HFA, obtained by setting $s(\mathbf{p}, \mathbf{k}, t)=0$ in Eq. (41). As mentioned above, in the latter case, the (spurious) HFA bound state does not interact with the FS pair excitations, even though it can merge with the continuum when one introduces a very short dephasing time.

In Fig. 3, the linear absorption lineshape (i.e., in the absence of pump) of the FES is compared to the HFA (without the dynamical FS response). The parameter values $g=0.4$ and $\Gamma=0.1 E_{F}$ were used, which were previously used to fit the experimental spectra in modulation doped quantum wells [50, 41]. For better visibility, we shifted the curves in order to compare their lineshapes. The linear absorption FES lineshape is consistent with that obtained in Ref. [50]. On the other hand, the HFA spectrum is characterized by the coexistence of the bound state and a continuum contribution due to the fact that, in 2D, a bound state exists even for an arbitrary weak attractive potential. We note that if one limits oneself to linear absorption, it is possible to artificially shorten the dephasing time $T_{2}=\Gamma^{-1}$, mainly determined by the hole recoil effects, by taking $\Gamma \simeq E_{M}$. Then the spurious discrete state and the continuum merge, and the discrepancy between the two linear absorption lineshapes decreases. This trick has been used for phenomenological fits of linear absorption experimental data. However, as we discuss below, in the nonlinear absorption case the differences in the transient spectra are significant so that the processes beyond HFA can be observed experimentally.

Let us turn to the time evolution of the pump-probe spectra. In Fig. 田 we show the nonlinear absorption spectra calculated by including the dynamical FS response [Fig. $4(\mathrm{a})$ ] and within the HFA [Fig. $4(\mathrm{~b})$ ] at a short time delay $\tau=-t_{p} / 2$. The main features of the spectrum are a pump-induced resonance bleaching, blueshift, and gain right below the onset of absorption. For offresonant pump, these transient effects vanish for positive time delays after the pump is gone, and persist for negative time delays shorter than the dephasing time $T_{2}=\Gamma^{-1}$. Similar features were also obtained for different 
values of the pump amplitude, duration, and detuning. They are mainly due to the broadening induced by the transient renormalization of the energy band dispersion [Eqs. (35) and (36)] when its duration $\sim t_{p}$ is shorter than the dephasing time (analogous to excitons and two-level systems).

Let us now turn to the role of the $e-h$ correlations. In Fig. 5 we compare the differential transmission spectrum calculated by including the dynamical FS response or within the HFA for long and short negative time delays. Note that, in PP spectroscopy, the experimentally measured differential transmission is given by Eq. (可) and in the weak signal regime, it reproduces the pump-induced changes in the probe absorption coefficient $\alpha(\omega, \tau): D S T(\omega, \tau) \propto-\Delta \alpha(\omega, \tau)$. Fig. 5(a) shows the results obtained for a long time delay, $\tau=-1.5 T_{2}=-15.0 E_{F}^{-1}$, in which case frequency domain oscillations are observed. These oscillations are similar to those seen in undoped semiconductors and two-level systems [179]; however, their amplitude in the FES case is reduced. On the other hand, as shown in Fig. 5(b), for time delays comparable to the pulse duration, $\tau=-0.1 T_{2}=-t_{p} / 2=-1.0 E_{F}^{-1}$, the main features are a blueshift and bleaching. In this case the $e-h$ correlations lead to a substantially larger width and asymmetric lineshape of the differential transmission spectrum. This comes from the different response of the FES to the pump-induced dispersion renormalizations when the $e-h$ correlations are accounted for. This is more clearly seen in Fig. 6, where the magnitude of the resonance decrease, evaluated at the peak frequency, is plotted as a function of $\tau$. Clearly, the bleaching of the FES peak is substantially stronger when the dynamical FS response is included than in the HFA case. Note that for $|\tau| \sim \Gamma^{-1}$ the FES resonance is actually enhanced by the pump, as can be seen more clearly in Fig. 7. The time dependence of the resonance bleaching is strikingly different in the two cases. In the HFA case, the $|D S T(\omega, \tau)|$ evaluated at the instantaneous peak frequency decays over a time scale $|\tau| \sim \Gamma^{-1}$, i.e. during the dephasing time. This is similar to results obtained for a two-level system with the same effective parameters. On the other hand, the decay of $|D S T(\omega, \tau)|$ at the peak frequency is much faster when we take into account the $e-h$ correlations. Note that the above results were obtained for off-resonant excitation. Under resonant conditions, a spectral hole is produced. In Fig. 8 we compare the resonance blueshifts, evaluated at the peak frequency, as a function of $\tau$. Again, a larger blueshift is predicted when the dynamical FS response is included. This suggests that in the experiment of Ref. [82], where similar blueshifts were observed in two quantum well samples (one MDQW with a FES and one undoped sample 
with a 2D-exciton) the effective parameters were larger in the latter case, due to the absence of screening and exciton-exciton interaction effects.

In order to gain qualitative understanding of the role of the $e-h$ correlations, let us for a moment neglect the momentum dependence of the pump-renormalization of the band dispersion and the phase space filling effects and consider the bleaching caused by a rigid semiconductor band shift $\Delta E_{g}(t)$, obtained from the pump-induced self-energies, Eqs. (35) and (36), evaluated at the bottom of the band (note that $\Delta E_{g}(t)$ lasts for the duration of pulse). Within this model, the pump excitation has no effect on the $e^{-} h$ scattering amplitude $s(\mathbf{p}, \mathbf{k}, t)$ [see Eq. (39)]. It is thus convenient to factorize the effects of the rigid band shift on the $e-h$ wavefunction $\Phi_{\mathbf{p}}\left(t, t^{\prime}\right)$ :

$$
\Phi_{\mathbf{p}}\left(t, t^{\prime}\right)=e^{-i \int_{t^{\prime}}^{t} \Delta E_{g}\left(t^{\prime \prime}\right) d t^{\prime \prime}} \tilde{\Phi}_{\mathbf{p}}\left(t, t^{\prime}\right) .
$$

This relation is general and defines $\tilde{\Phi}_{\mathbf{p}}\left(t, t^{\prime}\right)$, which does not depend on $\Delta E_{g}(t)$. In the special case of a rigid shift, $\tilde{\Phi}_{\mathbf{p}}\left(t, t^{\prime}\right)$ coincides with $\Phi_{\mathbf{p}}^{0}\left(t-t^{\prime}\right)$ describing the propagation of the probe-photoexcited $e-h$ pair in the absence of the pump pulse. By substituting into Eq. (58) the long-time asymptotic expression [163 $\tilde{\Phi}_{\mathbf{p}}\left(t, t^{\prime}\right)=\Phi_{\mathbf{p}}^{0}\left(t-t^{\prime}\right) \propto\left[i\left(t-t^{\prime}\right) E_{F}\right]^{\beta-1}$ that gives the linear absorption spectrum of the FES at $\omega \simeq E_{F}$, and substituting the resulting $\Phi_{\mathbf{p}}\left(t, t^{\prime}\right)$ into Eq. (44), we obtain a simple analytic expression for the effect of a pump-induced rigid band shift on the nonlinear absorption spectrum:

$$
\alpha(\omega) \propto \operatorname{Re} \int_{\tau}^{\infty} d t e^{i(\omega+i \Gamma)(t-\tau)-i \int_{\tau}^{t} \Delta E_{g}\left(t^{\prime}\right) d t^{\prime}}\left[i(t-\tau) E_{F}\right]^{\beta-1} .
$$

For $\Delta E_{g}(t)=0$ one, of course, recovers the linear FES absorption in the vicinity of the Fermi edge [163]. For $\beta=0$, Eq. (59) gives the absorption of the non-interacting continuum.

The physics of the FES can be seen from Eq. (59). For $\beta=1$, this gives a discrete Lorentzian peak corresponding to the HFA bound state. However, during the optical transition, the $e-h$ pair interacts with the FS electrons, leading to the readjustment of the FS density profile via the scattering of FS pairs. This results in the broadening of the discrete HFA bound state, which is governed by the time evolution of the FS. Such time evolution is unadiabatic due to the low-energy FS pairs, which leads to the characteristic power-law time dependence of the broadening factor in Eq. (59). The interaction with the FS-pairs determines the exponent, $0 \leq \beta \leq 1$, of the latter, which leads to a non-Lorentzian lineshape in the frequency domain 
and a non-exponential decay in the time domain. A detailed discussion of the above physics and the analogy to phonon sidebands and collision broadening may be found in Ref. [163]. However, the CCE must be used in order to calculate the spectrum at all frequencies (and not just asymptotically close to $E_{F}$ as with Eq. (59)) and, most importantly, to describe the nonequilibrium $\mathrm{FS}$ and $e-h$ pair response to the time-dependent increase in the effective mass/density of states, not included in Eq. (59).

The resonance bleaching obtained from Eq. (59) as a function of $\tau$, is shown in Fig. 6(b) for $\beta=0.6$, corresponding to the value of the parameters used in Fig. 6(a) $(g=0.4)$, together with the HFA result $(\beta=1)$. Comparing Figs. 6(a) and (b), one can see that the rigid band shift approximation qualitatively accounts for the dynamics, but that there are strong discrepancies (see vertical scales), whose origin is discussed below. Both the magnitude and the time-dependence of the bleaching depends critically on the value of $\beta$, which characterizes the interaction of the photoexcited $e-h$ pair with the FS excitations. Because of such coupling, many polarization components are excited in the case of the continuum FES resonance, and it is their interference that governs the dynamics of the PP signal. Such interference is also responsible for the resonance enhancement and differential transmission oscillations at $\tau<0$ shown in Figs. 5 and 7 . As $\beta$ increases the interference effects are suppressed because the energy width of the continuum states contributing to the FES narrows. In fact, this energy width is directly related to that of the linear absorption resonance. This is clearly seen in Fig. 9 where we show the effect of increasing $g$ on the dynamics of the bleaching. It becomes more bound-state-like as, with increasing $g$, the FES resonance becomes narrower. On the other hand, in the HFA case, the decay rate is $\sim T_{2}=\Gamma^{-1}$, i. e. it is independent on $g$, when $E_{M}$ becomes smaller than $\Gamma$, while for $E_{M} \simeq \Gamma$, the contribution of the continuum states produce a faster decay.

Although the transient rigid band shift approximation, Eq. (59), explains some of the features of the dynamics of the bleaching, it strongly overestimates its magnitude. This is because Eq. (59) neglects the response of the many-body system to the pump-induced renormalization of the band's dispersion. Such a transient change in the dispersion, which can be viewed as an increase in the density of states/effective mass for the duration of the pump, is important because it results in an enhancement of the $e-h$ scattering. For example, in the case of monochromatic excitation, this leads to the change in the exponent $\beta$ of the broadening prefactor in the integrand of Eq. (59), as 
discussed Section 5.1. For the short-pulse case, it is not possible to describe analytically the effect of the pump on the $e-h$ scattering processes, due to the non-equilibrium unadiabatic FS response. The latter can however be described in a simple way with the numerical solution of Eqs. (39) and (41), which is presented in Figs. 10 and 11 and discussed below.

In order to show the role of the pump-induced renormalization of the band dispersion in the presense of the dynamical FS response, we plot in Fig. 10 the function

$$
F(\omega, \tau)=\operatorname{Im} \sum_{p>k_{F}} \tilde{\Phi}_{\mathbf{p}}(\omega, \tau)
$$

where $\tilde{\Phi}_{\mathbf{p}}(\omega, \tau)$ is the Fourier transform of $\tilde{\Phi}_{\mathbf{p}}(t, \tau)$ defined by Eq. (58). Note that, in the presence of the band dispersion renormalization, the wavefunction $\tilde{\Phi}_{\mathbf{p}}$ (which is independent of $\left.\Delta E_{g}(t)\right)$ no longer coincides with $\Phi_{\mathbf{p}}^{0}$ as in Eq. (59). As can be seen in Fig. 10(a), when the $e-h$ correlations are taken into account, the pump-induced redistribution of oscillator strength between the states of the continuum that contribute to the resonance manifests itself as a dynamical redshift. This shift opposes the rigid band blueshift $\Delta E_{g}(t)$ (when the latter is included). At the same time, the resonance strength is enhanced significantly. The latter effect originates from the interplay between the transient increase in the effective mass/density of states of the photoexcited $e-h$ pair and the "dressing" of this pair with the FS excitations [described by the effective potential $\tilde{V}(\mathbf{p}, t)$ in Eq. (41)]. In contrast, such an oscillator strength enhancement is suppressed in the HFA (which neglects the $e-h$ correlations), as seen in Fig. 10(b), in which case the main feature is the redshift of the resonance due to the pump-induced increase of the binding energy $E_{M}$ coming from the transient increase in the effective mass.

In Fig. 11 the effect of the renormalization of the band dispersion on the nonlinear absorption spectrum is shown. The optically-induced increase in the $e-h$ interactions enhances significantly the strength of the FES and compensates part of the bleaching induced by the rigid band shift. A smaller enhancement is also seen in the HFA, where the pump-induced increase in the binding energy $E_{M}$ competes with the effects of the bandgap renormalization.

\subsection{Ultrafast Dynamics of the FES-Exciton Hybrid}

In this section we present the results for the evolution of the PP spectra

of the FES-exciton hybrid [180]. The spectra in Ref. [180] were obtained 
by the numerical solution of the coupled equations (54) and (53), with the time-dependent band dispersions $\epsilon_{i j \mathbf{k}}^{c}(t)$ and $\epsilon_{\mathbf{k}}^{v}(t)$. In typical MDQW's, the intersubband scattering strength characterized by the screened potential $v_{12}$ is much smaller than the intrasubband scattering strength characterized by $v_{i i}$ (a value $v_{12} / v_{11} \simeq 0.2$ was deduced from the fit to the linear absorption spectrum in [171]). As discussed in the previous section, in the absence of coupling $\left(v_{12}=0\right)$, the different nature of the exciton and FES leads to distinct dynamics under ultrafast excitation. In the presence of the coupling, one should expect new effects coming from the interplay of this difference and the intersubband scattering that hybridizes the two resonances. Indeed, it was demonstrated in Ref. [180] that, at negative time delays, the PP spectrum undergoes a drastic transformation due to a transient light-induced redistribution of the oscillator strength between the FES and the exciton. We discuss this at length in this section and show that such a redistribution is a result of the dynamical FS response to the pump pulse. In fact, the ultrafast PP spectra of the FES-exciton hybrid can serve as an experimental test of the difference between the FES and exciton dynamics.

The calculations in Ref. [180] were performed at zero temperature for below-resonant pump with detuning $\Omega \sim E_{F}$ and duration $t_{p} E_{F} / \hbar=2.0$, and by adopting the typical values of parameters $v_{12} / v_{11}=0.2, \Gamma=0.1 E_{F}$, and $v_{11} \mathcal{N}=0.3, \mathcal{N}$ being the density of states, previously extracted from fits to the linear absorption spectra [171, 43] $\left(E_{F} \sim 15-20 \mathrm{meV}\right.$ in typical GaAs/GaAlAs QW's [76, 82]). Note, however, that similar results were also obtained for a broad range of parameter values. In Fig. 12(a) we plot the nonlinear absorption spectra at different negative time delays $\tau<0$. For better visibility, the curves are shifted vertically with decreasing $|\tau|$ (the highest curve represents the linear absorption spectrum). For the chosen value of the subband separation $\Delta$, the FES and excitonic components of the hybrid are distinguishable in the linear absorption spectrum, with the FES peak carrying larger oscillator strength. It can be seen that, at short $\tau<0$, the oscillator strength is first transferred to the exciton and then, with further increase in $|\tau|$, back to the FES. At the same time, both peaks experience a blueshift, which is larger for the FES than for the exciton peak because the ac-Stark effect for the exciton is weaker due to the subband separation $\Delta$ and the correlation effects.

The transient exchange of oscillator strength originates from the different nature of the FES and exciton components of the hybrid. At negative time delays, the time-evolution of the exciton is governed by its dephasing time, 
which is essentially determined by the homogeneous broadening $\Gamma$ (in doped systems the exciton-exciton correlations do not play a significant role due to the screening). The pump pulse first leads to a bleaching of the exciton peak, which then recovers its strength at $|\tau| \sim \hbar / \Gamma$. On the other hand, since the FES is a many-body continuum resonance, (i) the bleaching of the FES peak is stronger, and (ii) the polarization decay of the FES is determined not by $\Gamma$, but by the scattering with the low-lying FS excitations. This leads to much faster dynamics, roughly determined by the inverse Coulomb energy $E_{M}$ (see discussion in the previous section). However, the time-evolution of the hybrid spectrum is not a simple superposition of the dynamics of its components. Indeed, the pump-induced self-energies lead to the flattening of the subbands or, to the first approximation, to a time-dependent increase in the effective mass (and hence the density of states), which in turn increases the $e-h$ scattering [157]. Important is, however, that, due to the subband separation and different nature of the resonances, such an increase is stronger for the FES. Therefore, the effect of the pump is to reduce the excitonic enhancement of the FES peak (coming from the resonant scattering of the photoexcited electron by the exciton level) as compared to the linear absorption case, resulting in the oscillator strength transfer from the FES back to exciton. In fact, such a transfer is strong even for smaller $\Delta$ [see Fig. 12(b)]. It should be emphasized that the above feature cannot be captured within the HFA. Indeed, the latter approximates the FES by a bound state and thus neglects the difference between the FES and exciton dynamics originating from the unadiabatic response of the FS to the change in the $e-h$ correlations. This is demonstrated in Fig. 12(c) where we show the spectra obtained without the FS dynamical response, i.e., by setting $s_{i j}=0$. Although in that case both peaks show blue shift and broadening, there is no significant transfer of oscillator strength

This concludes our discussion of the coherent nonlinear response of the FES. In the rest of this article, we will review the role of size-dependent correlation effects, due to quasiparticle scattering via surface collective modes, on the ultrafast dynamics of the SP resonance in small metal nanoparticles. 


\section{Quasiparticle Scattering with Surface Col- lective Modes in Metal Nanoparticles}

\subsection{Electron-Electron Interactions in Metal Nanopar- ticles}

Before we proceed with the ultrafast dynamics, we discuss in this section the effect of the surface collective excitations on the $e-e$ interactions in a spherical metal particle. In particular, we present a detailed derivation of the dynamically screened Coulomb potential [152, 153] by generalizing a method previously developed for calculations of local field corrections to the optical fields [181.

The potential $U\left(\omega ; \mathbf{r}, \mathbf{r}^{\prime}\right)$ at point $\mathbf{r}$ arising from an electron at point $\mathbf{r}^{\prime}$ is determined by the equation 43.

$$
U\left(\omega ; \mathbf{r}, \mathbf{r}^{\prime}\right)=u\left(\mathbf{r}-\mathbf{r}^{\prime}\right)+\int d \mathbf{r}_{1} d \mathbf{r}_{2} u\left(\mathbf{r}-\mathbf{r}_{1}\right) \Pi\left(\omega ; \mathbf{r}_{1}, \mathbf{r}_{2}\right) U\left(\omega ; \mathbf{r}_{2}, \mathbf{r}^{\prime}\right)
$$

where $u\left(\mathbf{r}-\mathbf{r}^{\prime}\right)=e^{2}\left|\mathbf{r}-\mathbf{r}^{\prime}\right|^{-1}$ is the unscreened Coulomb potential and $\Pi\left(\omega ; \mathbf{r}_{1}, \mathbf{r}_{2}\right)$ is the polarization operator. There are three contributions to $\Pi$, arising from the polarization of the conduction electrons, the $d$-electrons, and the medium surrounding the nanoparticles: $\Pi=\Pi_{c}+\Pi_{d}+\Pi_{m}$. It is useful to rewrite Eq. (61) in the "classical" form

$$
\nabla \cdot(\mathbf{E}+4 \pi \mathbf{P})=4 \pi e^{2} \delta\left(\mathbf{r}-\mathbf{r}^{\prime}\right)
$$

where $\mathbf{E}\left(\omega ; \mathbf{r}, \mathbf{r}^{\prime}\right)=-\nabla U\left(\omega ; \mathbf{r}, \mathbf{r}^{\prime}\right)$ is the screened Coulomb field and $\mathbf{P}=$ $\mathbf{P}_{c}+\mathbf{P}_{d}+\mathbf{P}_{m}$ is the electric polarization vector, related to the potential $U$ as

$$
\nabla \mathbf{P}\left(\omega ; \mathbf{r}, \mathbf{r}^{\prime}\right)=-e^{2} \int d \mathbf{r}_{1} \Pi\left(\omega ; \mathbf{r}, \mathbf{r}_{1}\right) U\left(\omega ; \mathbf{r}_{1}, \mathbf{r}^{\prime}\right) .
$$

In the random phase approximation (RPA), the intraband polarization operator is given by

$$
\Pi_{c}\left(\omega ; \mathbf{r}, \mathbf{r}^{\prime}\right)=\sum_{\alpha \alpha^{\prime}} \frac{f\left(E_{\alpha}^{c}\right)-f\left(E_{\alpha^{\prime}}^{c}\right)}{E_{\alpha}^{c}-E_{\alpha^{\prime}}^{c}+\omega+i 0} \psi_{\alpha}^{c}(\mathbf{r}) \psi_{\alpha^{\prime}}^{c *}(\mathbf{r}) \psi_{\alpha}^{c *}\left(\mathbf{r}^{\prime}\right) \psi_{\alpha^{\prime}}^{c}\left(\mathbf{r}^{\prime}\right),
$$

where $E_{\alpha}^{c}$ and $\psi_{\alpha}^{c}$ are the single-electron eigenenergies and eigenfunctions in the nanoparticle, and $f(E)$ is the Fermi-Dirac distribution (we set $\hbar=1$ ). Since we are interested in frequencies much larger than the single-particle 
level spacing, $\Pi_{c}(\omega)$ can be expanded in terms of $1 / \omega$. For the real part, $\Pi_{c}^{\prime}(\omega)$, we obtain in the leading order

$$
\Pi_{c}^{\prime}\left(\omega ; \mathbf{r}, \mathbf{r}_{1}\right)=-\frac{1}{m \omega^{2}} \nabla\left[n_{c}(\mathbf{r}) \nabla \delta\left(\mathbf{r}-\mathbf{r}_{1}\right)\right]
$$

where $n_{c}(\mathbf{r})$ is the conduction electron density. In the following we assume, for simplicity, a step density profile, $n_{c}(\mathbf{r})=\bar{n}_{c} \theta(R-r)$, where $\bar{n}_{c}$ is the average density. The leading contribution to the imaginary part, $\Pi_{c}^{\prime \prime}(\omega)$, is proportional to $\omega^{-3}$, so that $\Pi_{c}^{\prime \prime}(\omega) \ll \Pi_{c}^{\prime}(\omega)$.

By using Eqs. (65) and (63), we obtain a familiar expression for $\mathbf{P}_{c}$ at high frequencies,

$$
\mathbf{P}_{c}\left(\omega ; \mathbf{r}, \mathbf{r}^{\prime}\right)=\frac{e^{2} n_{c}(\mathbf{r})}{m \omega^{2}} \nabla U\left(\omega ; \mathbf{r}, \mathbf{r}^{\prime}\right)=\theta(R-r) \chi_{c}(\omega) \mathbf{E}\left(\omega ; \mathbf{r}, \mathbf{r}^{\prime}\right)
$$

where $\chi_{c}(\omega)=-e^{2} \bar{n}_{c} / m \omega^{2}$ is the conduction electron susceptibility. Note that, for a step density profile, $\mathbf{P}_{c}$ vanishes outside the particle. The $d$-band and dielectric medium contributions to $\mathbf{P}$ are also given by similar relations,

$$
\begin{gathered}
\mathbf{P}_{d}\left(\omega ; \mathbf{r}, \mathbf{r}^{\prime}\right)=\theta(R-r) \chi_{d}(\omega) \mathbf{E}\left(\omega ; \mathbf{r}, \mathbf{r}^{\prime}\right), \\
\mathbf{P}_{m}\left(\omega ; \mathbf{r}, \mathbf{r}^{\prime}\right)=\theta(r-R) \chi_{m} \mathbf{E}\left(\omega ; \mathbf{r}, \mathbf{r}^{\prime}\right),
\end{gathered}
$$

where $\chi_{i}=\left(\epsilon_{i}-1\right) / 4 \pi, i=d, m$ are the corresponding susceptibilities and the step functions account for the boundary conditions [182. Using Eqs. (66)(68), one can write a closed-form equation for $U\left(\omega ; \mathbf{r}, \mathbf{r}^{\prime}\right)$. Using Eq. (63), the second term of Eq. (61) can be presented as $-e^{-2} \int d \mathbf{r}_{1} u\left(\mathbf{r}-\mathbf{r}_{1}\right) \nabla \cdot \mathbf{P}\left(\omega ; \mathbf{r}_{1}, \mathbf{r}^{\prime}\right)$. Substituting the above expressions for $\mathbf{P}$, we then obtain after integration by parts

$$
\begin{aligned}
\epsilon(\omega) U\left(\omega ; \mathbf{r}, \mathbf{r}^{\prime}\right)= & \frac{e^{2}}{\left|\mathbf{r}-\mathbf{r}^{\prime}\right|}+\int d \mathbf{r}_{1} \nabla_{1} \frac{1}{\left|\mathbf{r}-\mathbf{r}_{1}\right|} \cdot \nabla_{1}[\theta(R-r) \chi(\omega) \\
& \left.+\theta(r-R) \chi_{m}\right] U\left(\omega ; \mathbf{r}_{1}, \mathbf{r}^{\prime}\right) \\
& +i \int d \mathbf{r}_{1} d \mathbf{r}_{2} \frac{e^{2}}{\left|\mathbf{r}-\mathbf{r}_{1}\right|} \Pi_{c}^{\prime \prime}\left(\omega ; \mathbf{r}_{1}, \mathbf{r}_{2}\right) U\left(\omega ; \mathbf{r}_{2}, \mathbf{r}^{\prime}\right)
\end{aligned}
$$

with

$$
\epsilon(\omega) \equiv 1+4 \pi \chi(\omega)=\epsilon_{d}(\omega)-\omega_{p}^{2} / \omega^{2},
$$

$\omega_{p}^{2}=4 \pi e^{2} \bar{n}_{c} / m$ being the plasmon frequency in the conduction band. The last term in the rhs of Eq. (69), proportional to $\Pi_{c}^{\prime \prime}(\omega)$, can be regarded as a 
small correction. To solve Eq. (69), we first eliminate the angular dependence by expanding $U\left(\omega ; \mathbf{r}, \mathbf{r}^{\prime}\right)$ in spherical harmonics, $Y_{L M}(\hat{\mathbf{r}})$, with coefficients $U_{L M}\left(\omega ; r, r^{\prime}\right)$. Using the corresponding expansion of $\left|\mathbf{r}-\mathbf{r}^{\prime}\right|^{-1}$ with coefficients $Q_{L M}\left(r, r^{\prime}\right)=\frac{4 \pi}{2 L+1} r^{-L-1} r^{\prime L}$ (for $r>r^{\prime}$ ), we get the following equation for $U_{L M}\left(\omega ; r, r^{\prime}\right)$ :

$$
\begin{aligned}
\epsilon(\omega) U_{L M}\left(\omega ; r, r^{\prime}\right)= & Q_{L M}\left(r, r^{\prime}\right)+4 \pi\left[\chi(\omega)-\chi_{m}\right] \frac{L+1}{2 L+1}\left(\frac{r}{R}\right)^{L} U_{L M}\left(\omega ; R, r^{\prime}\right) \\
& +i e^{2} \sum_{L^{\prime} M^{\prime}} \int d r_{1} d r_{2} r_{1}^{2} r_{2}^{2} Q_{L M}\left(r, r_{1}\right) \Pi_{L M, L^{\prime} M^{\prime}}^{\prime \prime}\left(\omega ; r_{1}, r_{2}\right) \\
& \times U_{L^{\prime} M^{\prime}}\left(\omega ; r_{2}, r^{\prime}\right),
\end{aligned}
$$

where

$$
\Pi_{L M, L^{\prime} M^{\prime}}^{\prime \prime}\left(\omega ; r_{1}, r_{2}\right)=\int d \hat{\mathbf{r}}_{1} d \hat{\mathbf{r}}_{2} Y_{L M}^{*}\left(\hat{\mathbf{r}}_{1}\right) \Pi_{c}^{\prime \prime}\left(\omega ; \mathbf{r}_{1}, \mathbf{r}_{2}\right) Y_{L^{\prime} M^{\prime}}\left(\hat{\mathbf{r}}_{2}\right),
$$

are the coefficients of the multipole expansion of $\Pi_{c}^{\prime \prime}\left(\omega ; \mathbf{r}_{1}, \mathbf{r}_{2}\right)$. For $\Pi_{c}^{\prime \prime}=0$, the solution of Eq. (71) can be presented in the form

$$
U_{L M}\left(\omega ; r, r^{\prime}\right)=a(\omega) e^{2} Q_{L M}\left(r, r^{\prime}\right)+b(\omega) \frac{4 \pi e^{2}}{2 L+1} \frac{r^{L} r^{\prime L}}{R^{2 L+1}},
$$

with frequency-dependent coefficients $a$ and $b$. Since $\Pi_{c}^{\prime \prime}(\omega) \ll \Pi_{c}^{\prime}(\omega)$ for the relevant frequencies, the solution of Eq. (71) in the presence of the last term can be written in the same form as Eq. (73), but with modified $a(\omega)$ and $b(\omega)$. Substituting Eq. (73) into Eq. (71), we obtain after lengthy algebra in the lowest order in $\Pi_{c}^{\prime \prime}$

$$
a(\omega)=\epsilon^{-1}(\omega), \quad b(\omega)=\epsilon_{L}^{-1}(\omega)-\epsilon^{-1}(\omega),
$$

where

$$
\epsilon_{L}(\omega)=\frac{L}{2 L+1} \epsilon(\omega)+\frac{L+1}{2 L+1} \epsilon_{m}+i \epsilon_{c L}^{\prime \prime}(\omega),
$$

is the effective dielectric function, whose zero, $\epsilon_{L}^{\prime}\left(\omega_{L}\right)=0$, determines the frequency of the collective surface excitation with angular momentum $L$ 103],

$$
\omega_{L}^{2}=\frac{L \omega_{p}^{2}}{L \epsilon_{d}^{\prime}\left(\omega_{L}\right)+(L+1) \epsilon_{m}} .
$$


In Eq. (75), $\epsilon_{c L}^{\prime \prime}(\omega)$ characterizes the damping of the $L$-pole collective mode by single-particle excitations, and is given by

$$
\epsilon_{c L}^{\prime \prime}(\omega)=\frac{4 \pi^{2} e^{2}}{(2 L+1) R^{2 L+1}} \sum_{\alpha \alpha^{\prime}}\left|M_{\alpha \alpha^{\prime}}^{L M}\right|^{2}\left[f\left(E_{\alpha}^{c}\right)-f\left(E_{\alpha^{\prime}}^{c}\right)\right] \delta\left(E_{\alpha}^{c}-E_{\alpha^{\prime}}^{c}+\omega\right),
$$

where $M_{\alpha \alpha^{\prime}}^{L M}$ are the matrix elements of $r^{L} Y_{L M}(\hat{\mathbf{r}})$. Due to the momentum non-conservation in a nanoparticle, the matrix elements are finite, which leads to the size-dependent width of the $L$-pole mode [107, 181]:

$$
\gamma_{L}=\frac{2 L+1}{L} \frac{\omega^{3}}{\omega_{p}^{2}} \epsilon_{c L}^{\prime \prime}(\omega)
$$

For $\omega \sim \omega_{L}$, one can show that the width, $\gamma_{L} \sim v_{F} / R$, is independent of $\omega$. Note that, in noble metal particles, there is an additional $d$-electron contribution to the imaginary part of $\epsilon_{L}(\omega)$ at frequencies above the onset $\Delta$ of the interband transitions.

Putting everything together, we arrive at the following expression for the dynamically-screened interaction potential in a nanoparticle:

$$
U\left(\omega ; \mathbf{r}, \mathbf{r}^{\prime}\right)=\frac{u\left(\mathbf{r}-\mathbf{r}^{\prime}\right)}{\epsilon(\omega)}+\frac{e^{2}}{R} \sum_{L M} \frac{4 \pi}{2 L+1} \frac{1}{\tilde{\epsilon}_{L}(\omega)}\left(\frac{r r^{\prime}}{R^{2}}\right)^{L} Y_{L M}(\hat{\mathbf{r}}) Y_{L M}^{*}\left(\hat{\mathbf{r}}^{\prime}\right),
$$

with $\tilde{\epsilon}_{L}^{-1}(\omega)=\epsilon_{L}^{-1}(\omega)-\epsilon^{-1}(\omega)$. Equation (79) represents a generalization of the plasmon pole approximation to spherical metal particles. The two contributions to the rhs originate from two types of dynamical screening. The first describes the usual bulk-like screening of the Coulomb potential by the electrons inside the particle. The second contribution is a new effective interaction induced by the surface: the potential of an electron inside the nanoparticle excites high-frequency surface collective modes, which in turn act as image charges that interact with the second electron. It should be emphasized that, unlike in the case of the optical fields, the surface-induced dynamical screening of the Coulomb potential is size-dependent.

Note that the excitation energies of the surface collective modes are lower than the bulk plasmon energy, also given by Eq. (76) but with $\epsilon_{m}=0$. As discussed below, this opens up new channels of quasiparticle scattering. 


\subsection{Conduction Electron Scattering}

In this section, we present the calculation of the rates of electron scattering in the conduction band accompanied by the emission of surface collective modes and discuss its possible experimental manifestations. In the first order in the surface-induced potential, given by the second term in the rhs of Eq. (79), the corresponding scattering rate can be obtained from the Matsubara selfenergy 443

$$
\Sigma_{\alpha}^{c}(i \omega)=-\frac{1}{\beta} \sum_{i \omega^{\prime}} \sum_{L M} \sum_{\alpha^{\prime}} \frac{4 \pi e^{2}}{(2 L+1) R^{2 L+1}} \frac{\left|M_{\alpha \alpha^{\prime}}^{L M}\right|^{2}}{\tilde{\epsilon}_{L}\left(i \omega^{\prime}\right)} G_{\alpha^{\prime}}^{c}\left(i \omega^{\prime}+i \omega\right),
$$

where $G_{\alpha}^{c}=\left(i \omega-E_{\alpha}^{c}\right)^{-1}$ is the non-interacting Green function of the conduction electron. Here the matrix elements $M_{\alpha \alpha^{\prime}}^{L M}$ are calculated with the one-electron wave functions $\psi_{\alpha}^{c}(\mathbf{r})=R_{n l}(r) Y_{l m}(\hat{\mathbf{r}})$. Since $|\alpha\rangle$ and $\left|\alpha^{\prime}\right\rangle$ are the initial and final states of the scattered electron, the main contribution to the $L$ th term of the angular momentum sum in Eq. (80) will come from electron states with energy difference $E_{\alpha}-E_{\alpha^{\prime}} \sim \omega_{L}$. Therefore, $M_{\alpha \alpha^{\prime}}^{L M}$ can be expanded in terms of the small parameter $E_{0} /\left|E_{\alpha}^{c}-E_{\alpha^{\prime}}^{c}\right| \sim E_{0} / \omega_{L}$, where $E_{0}=\left(2 m R^{2}\right)^{-1}$ is the characteristic confinement energy. The leading term can be obtained by using the following procedure 107, 181. We present $M_{\alpha \alpha^{\prime}}^{L M}$ as

$$
M_{\alpha \alpha^{\prime}}^{L M}=\left\langle c, \alpha\left|r^{L} Y_{L M}(\hat{\mathbf{r}})\right| c, \alpha^{\prime}\right\rangle=\frac{\left\langle c, \alpha\left|\left[H,\left[H, r^{L} Y_{L M}(\hat{\mathbf{r}})\right]\right]\right| c, \alpha^{\prime}\right\rangle}{\left(E_{\alpha}^{c}-E_{\alpha^{\prime}}^{c}\right)^{2}}
$$

where $H=H_{0}+V(r)$ is the Hamiltonian of an electron in a nanoparticle with confining potential $V(r)=V_{0} \theta(r-R)$. Since $\left[H, r^{L} Y_{L M}(\hat{\mathbf{r}})\right]=$ $-\frac{1}{m} \nabla\left[r^{L} Y_{L M}(\hat{\mathbf{r}})\right] \cdot \nabla$, the numerator in Eq. (81) contains a term proportional to the gradient of the confining potential, which peaks sharply at the surface. The corresponding contribution to the matrix element describes the surface scattering of an electron making the $L$-pole transition between the states $|c, \alpha\rangle$ and $\left|c, \alpha^{\prime}\right\rangle$, and gives the dominant term of the expansion. Thus, in the leading order in $\left|E_{\alpha}^{c}-E_{\alpha^{\prime}}^{c}\right|^{-1}$, we obtain

$$
\begin{aligned}
M_{\alpha \alpha^{\prime}}^{L M}= & \frac{\left\langle c, \alpha\left|\nabla\left[r^{L} Y_{L M}(\hat{\mathbf{r}})\right] \cdot \nabla V(r)\right| c, \alpha^{\prime}\right\rangle}{m\left(E_{\alpha}^{c}-E_{\alpha^{\prime}}^{c}\right)^{2}} \\
& =\frac{L R^{L+1}}{m\left(E_{\alpha}^{c}-E_{\alpha^{\prime}}^{c}\right)^{2}} V_{0} R_{n l}(R) R_{n^{\prime} l^{\prime}}(R) \varphi_{l m, l^{\prime} m^{\prime}}^{L M}
\end{aligned}
$$


with $\varphi_{l m, l^{\prime} m^{\prime}}^{L M}=\int d \hat{\mathbf{r}} Y_{l m}^{*}(\hat{\mathbf{r}}) Y_{L M}(\hat{\mathbf{r}}) Y_{l^{\prime} m^{\prime}}(\hat{\mathbf{r}})$. Note that, for $L=1$, Eq. 82 becomes exact. For electron energies close to the Fermi level, $E_{n l}^{c} \sim E_{F}$, the radial quantum numbers are large, and the product $V_{0} R_{n l}(R) R_{n^{\prime} l^{\prime}}(R)$ can be evaluated by using semiclassical wave-functions. In the limit $V_{0} \rightarrow \infty$, this product is given by [107 $2 \sqrt{E_{n l}^{c} E_{n^{\prime} l^{\prime}}^{c}} / R^{3}$, where $E_{n l}^{c}=\pi^{2}(n+l / 2)^{2} E_{0}$ is the electron eigenenergy for large $n$. Substituting this expression into Eq. (82) and then into Eq. (80), we obtain

$$
\begin{aligned}
\Sigma_{\alpha}^{c}(i \omega)= & -\frac{1}{\beta} \sum_{i \omega^{\prime}} \sum_{L} \sum_{n^{\prime} l^{\prime}} C_{l l^{\prime}}^{L} \frac{4 \pi e^{2}}{(2 L+1) R} \frac{E_{n l}^{c} E_{n^{\prime} l^{\prime}}^{c}}{\left(E_{n l}^{c}-E_{n^{\prime} l^{\prime}}^{c}\right)^{4}} \\
& \times \frac{\left(4 L E_{0}\right)^{2}}{\tilde{\epsilon}_{L}\left(i \omega^{\prime}\right)} G_{\alpha^{\prime}}^{c}\left(i \omega^{\prime}+i \omega\right),
\end{aligned}
$$

with

$$
C_{l l^{\prime}}^{L}=\sum_{M, m^{\prime}}\left|\varphi_{l m, l^{\prime} m^{\prime}}^{L M}\right|^{2}=\frac{(2 L+1)\left(2 l^{\prime}+1\right)}{8 \pi} \int_{-1}^{1} d x P_{l}(x) P_{L}(x) P_{l^{\prime}}(x),
$$

where $P_{l}(x)$ are Legendre polynomials; we used properties of the spherical harmonics in the derivation of Eq. (84). For $E_{n l}^{c} \sim E_{F}$, the typical angular momenta are large, $l \sim k_{F} R \gg 1$, and one can use the large- $l$ asymptotics of $P_{l}$; for the low multipoles of interest, $L \ll l$, the integral in Eq. (84) can be approximated by $\frac{2}{2 l^{\prime}+1} \delta_{l l^{\prime}}$. After performing the Matsubara summation, we obtain for the imaginary part of the self-energy that determines the electron scattering rate

$$
\operatorname{Im} \Sigma_{\alpha}^{c}(\omega)=-\frac{16 e^{2}}{R} E_{0}^{2} \sum_{L} \int d E g_{l}(E) \frac{L^{2} E E_{\alpha}^{c}}{\left(E_{\alpha}^{c}-E\right)^{4}} \operatorname{Im} \frac{N(E-\omega)+f(E)}{\tilde{\epsilon}_{L}(E-\omega)},
$$

where $N(E)$ is the Bose distribution and $g_{l}(E)$ is the density of states of a conduction electron with angular momentum $l$,

$$
g_{l}(E)=2 \sum_{n} \delta\left(E_{n l}^{c}-E\right) \simeq \frac{R}{\pi} \sqrt{\frac{2 m}{E}},
$$

where we replaced the sum over $n$ by an integral (the factor of 2 accounts for spin).

Each term in the sum in the rhs of Eq. (85) represents a channel of electron scattering mediated by a collective surface mode with angular momentum $L$. 
For low $L$, the difference between the energies of modes with successive values of $L$ is larger than their widths, so the different channels are well separated. Note that since all $\omega_{L}$ are smaller than the frequency of the bulk plasmon, one can replace $\tilde{\epsilon}_{L}(\omega)$ by $\epsilon_{L}(\omega)$ in the integrand of Eq. (85) for frequencies $\omega \sim \omega_{L}$.

Consider now the $L=1$ term in Eq. (85), which describes the SPmediated scattering channel. The main contribution to the integral comes from the SP pole in $\epsilon_{1}^{-1}(\omega)=3 \epsilon_{s}^{-1}(\omega)$, where $\epsilon_{s}(\omega)$ is the same as in Eq. (13). To evaluate the integral in Eq. (85), we can in the first approximation replace $\operatorname{Im} \epsilon_{s}^{-1}(\omega)$ by a Lorentzian,

$$
\begin{aligned}
\operatorname{Im} \frac{1}{\epsilon_{s}(\omega)}= & -\frac{\gamma_{s} \omega_{p}^{2} / \omega^{3}+\epsilon_{d}^{\prime \prime}(\omega)}{\left[\epsilon^{\prime}(\omega)+2 \epsilon_{m}\right]^{2}+\left[\gamma_{s} \omega_{p}^{2} / \omega^{3}+\epsilon_{d}^{\prime \prime}(\omega)\right]^{2}} \\
& \simeq-\frac{\omega_{s}^{2}}{\epsilon_{d}^{\prime}\left(\omega_{s}\right)+2 \epsilon_{m}} \frac{\omega_{s} \gamma}{\left(\omega^{2}-\omega_{s}^{2}\right)^{2}+\omega_{s}^{2} \gamma^{2}}
\end{aligned}
$$

where $\omega_{s} \equiv \omega_{1}=\omega_{p} / \sqrt{\epsilon_{d}^{\prime}\left(\omega_{s}\right)+2 \epsilon_{m}}$ and $\gamma=\gamma_{s}+\omega_{s} \epsilon_{d}^{\prime \prime}\left(\omega_{s}\right)$ are the SP frequency and width, respectively. For typical widths $\gamma \ll \omega_{s}$, the integral in Eq. (85) can be easily evaluated, yielding

$$
\operatorname{Im} \Sigma_{\alpha}^{c}(\omega)=-\frac{24 e^{2} \omega_{s} E_{0}^{2}}{\epsilon_{d}^{\prime}\left(\omega_{s}\right)+2 \epsilon_{m}} \frac{E_{\alpha}^{c} \sqrt{2 m\left(\omega-\omega_{s}\right)}}{\left(\omega-E_{\alpha}^{c}-\omega_{s}\right)^{4}}\left[1-f\left(\omega-\omega_{s}\right)\right] .
$$

Using the relation $e^{2} k_{F}\left[\epsilon_{d}^{\prime}\left(\omega_{s}\right)+2 \epsilon_{m}\right]^{-1}=3 \pi \omega_{s}^{2} / 8 E_{F}$, the SP-mediated scattering rate, $\gamma_{e}^{s}\left(E_{\alpha}^{c}\right)=-\operatorname{Im} \Sigma_{\alpha}^{c}\left(E_{\alpha}^{c}\right)$, takes the form

$$
\gamma_{e}^{s}(E)=9 \pi \frac{E_{0}^{2}}{\omega_{s}} \frac{E}{E_{F}}\left(\frac{E-\omega_{s}}{E_{F}}\right)^{1 / 2}\left[1-f\left(E-\omega_{s}\right)\right] .
$$

Recalling that $E_{0}=\left(2 m R^{2}\right)^{-1}$, we see that the scattering rate of a conduction electron is size-dependent: $\gamma_{e}^{s} \propto R^{-4}$. At $E=E_{F}+\omega_{s}$, the scattering rate jumps to the value $9 \pi\left(1+\omega_{s} / E_{F}\right) E_{0}^{2} / \omega_{s}$, and then increases with energy as $E^{3 / 2}$ (for $\omega_{s} \ll E_{F}$ ). This should be contrasted with the usual (bulk) plasmon-mediated scattering, originating from the first term in Eq. (79), in which case the rate decreases as $E^{-1 / 2}$ above the onset [43].

Note that the total electron scattering rate is the sum, $\gamma_{e}+\gamma_{e}^{s}$, of the $\mathrm{SP}$-mediated $\left(\gamma_{e}^{s}\right)$ and the bulk-like $\left(\gamma_{e}\right)$ scattering rates. In order to be observable, the former should exceed the latter. The typical size at which 
$\gamma_{e}^{s}$ becomes important can be estimated by equating $\gamma_{e}^{s}$ and the Fermi liquid

$e-e$ scattering rate [回], $\gamma_{e}(E)=\frac{\pi^{2} q_{T F}}{16 k_{F}} \frac{\left(E-E_{F}\right)^{2}}{E_{F}}$. For energies $E \sim E_{F}+\omega_{s}$, the two rates become comparable for

$$
\left(k_{F} R\right)^{2} \simeq 12 \frac{E_{F}}{\omega_{s}}\left(1+\frac{E_{F}}{\omega_{s}}\right)^{1 / 2}\left(\frac{k_{F}}{\pi q_{T F}}\right)^{1 / 2} .
$$

In the case of a copper nanoparticle with $\omega_{s} \simeq 2.2 \mathrm{eV}$, we obtain $k_{F} R \simeq 8$, which corresponds to a radius of $R \simeq 3 \mathrm{~nm}$. At the same time, in this energy range, the width $\gamma_{e}^{s}$ exceeds the mean level spacing $\delta$, so that the energy spectrum is still continuous. The strong size dependence of $\gamma_{e}^{s}$ indicates that, although $\gamma_{e}^{s}$ increases with energy slower than $\gamma_{e}$, the SP-mediated scattering should dominate for nanometer-sized particles. Note also that the size and energy dependences of the scattering in the different channels are similar: the rate of scattering via the $L$ th channel is given by Eq. (89) with $\omega_{s}$ replaced by $\omega_{L}$, Eq. (76), and the numerical factor 9 replaced by $3 L(2 L+1)$.

Concluding this section, we have shown that the $\mathrm{SP}$-mediated scattering is the dominant scattering mechanism of conduction electrons in nanometersized nanoparticles for energies larger than $\omega_{s}$ but smaller than $\omega_{p}$. The scattering rate in the $L$ th channel, $\gamma_{e}^{L}$, increases with energy, in sharp contrast with the bulk-plasmon-mediated scattering rate. The total scattering rate as a function of energy represents a series of steps at $E=\omega_{L}$, on top of a smooth energy increase. We expect that this new effect should be observable experimentally by measuring $e-e$ scattering rate in size-selected cluster beams in time-resolved two-photon photoemission spectrum [183.

\section{$6.3 \quad d$-Band Hole Scattering}

We now turn to the interband processes in noble metal particles and consider the scattering of a $d$-hole into the conduction band. The corresponding surface-induced potential, given by the Lth term in Eq. (79), has the form

$$
U_{L M}\left(\omega ; \mathbf{r}, \mathbf{r}^{\prime}\right)=\frac{4 \pi}{2 L+1} \frac{1}{\epsilon_{L}(\omega)} \frac{e^{2}}{R}\left(\frac{r r^{\prime}}{R^{2}}\right)^{L} Y_{L M}(\hat{\mathbf{r}}) Y_{L M}^{*}\left(\hat{\mathbf{r}}^{\prime}\right),
$$

where $\epsilon_{L}(\omega)$ is given by Eq. (75) With this potential, the $d$-hole self-energy is given by

$$
\Sigma_{\alpha}^{d}(i \omega)=-\frac{e^{2}}{R^{2 L+1}} \sum_{\alpha^{\prime}}\left|\tilde{M}_{\alpha \alpha^{\prime}}^{L M}\right|^{2} \frac{1}{\beta} \sum_{i \omega^{\prime}} \frac{G_{\alpha^{\prime}}^{c}\left(i \omega^{\prime}+i \omega\right)}{\epsilon_{L}\left(i \omega^{\prime}\right)},
$$


where $\tilde{M}_{\alpha \alpha^{\prime}}^{L M}=\left\langle c, \alpha\left|r^{L} Y_{L M}(\hat{\mathbf{r}})\right| d, \alpha^{\prime}\right\rangle$ is the interband transition matrix element [compare with Eq. (81)]. Since the final state energies in the conduction band are high (in the case of interest here, they are close to the Fermi level), the matrix element can be approximated by a bulk-like expression $\tilde{M}_{\alpha \alpha^{\prime}}^{L M}=\delta_{\alpha \alpha^{\prime}} \tilde{M}_{\alpha \alpha}^{L M}$, the corrections due to surface scattering being suppressed by a factor of $\left(k_{F} R\right)^{-1} \ll 1$.

The largest contribution to the self energy Eq. (92) comes from the dipole channel, $L=1$, mediated by the SP. In this case, after performing the frequency summation, we obtain for $\operatorname{Im} \Sigma_{\alpha}^{d}$

$$
\operatorname{Im} \Sigma_{\alpha}^{d}(\omega)=-\frac{9 e^{2} \mu^{2}}{m^{2}\left(E_{\alpha}^{c d}\right)^{2} R^{3}} \operatorname{Im} \frac{N\left(E_{\alpha}^{c}-\omega\right)+f\left(E_{\alpha}^{c}\right)}{\epsilon_{s}\left(E_{\alpha}^{c}-\omega\right)}
$$

where $E_{\alpha}^{c d}=E_{\alpha}^{c}-E_{\alpha}^{d}$ and $\mu$ is the interband dipole matrix element 152. We see that the scattering rate of a $d$-hole with energy $E_{\alpha}^{d}, \gamma_{h}^{s}\left(E_{\alpha}^{d}\right)=\operatorname{Im} \Sigma_{\alpha}^{d}\left(E_{\alpha}^{d}\right)$, has a strong $R^{-3}$ dependence on the nanoparticle size, which is, however, different from that of the intraband scattering, Eq. (89).

The most important difference between the interband and the intraband $\mathrm{SP}-$ mediated scattering rates lies in their dependence on energy. Since the surface-induced potential, Eq. (91), only allows for vertical (dipole) interband single-particle excitations, the phase space available for the scattering of a $d$-hole with energy $E_{\alpha}^{d}$ is restricted to a single final state in the conduction band, with energy $E_{\alpha}^{c}$. As a result of this restriction, the $d$-hole scattering rate, $\gamma_{h}^{s}\left(E_{\alpha}^{d}\right)$, exhibits a peak as the difference between the energies of final and initial states, $E_{\alpha}^{c d}=E_{\alpha}^{c}-E_{\alpha}^{d}$, approaches the SP frequency $\omega_{s}$ [see Eq. (93)]. In contrast, the energy dependence of $\gamma_{e}^{s}$ is smooth due the larger phase space available for scattering within the conduction band. This leads to the additional integral over final state energies in Eq. (85), which smears out the SP resonant enhancement of the intraband scattering.

As we show later, the fact that the scattering rate of a $d$-hole is dominated by the SP resonance affects strongly the nonlinear optical dynamics in small nanoparticles. This is the case, in particular, when the SP frequency, $\omega_{s}$, is close to the onset of interband transitions, $\Delta$, as, e.g., in copper and gold nanoparticles [103, 126, 127]. Indeed, if the optical pulse excites an $e-h$ pair with excitation energy $\omega$ close to $\Delta$, the $d$-hole can subsequently scatter into the conduction band by emitting a SP. According to Eq. (93), for $\omega \sim \omega_{s}$, such a scattering process should be resonantly enhanced. In order to have an observable effect on the absorption spectrum, the scattering 
rate of the photoexcited $d$-hole should be comparable (or larger) than that of the photoexcited electron. Close to $E_{F}$, the electron scattering in the conduction band comes from a two-quasiparticle process; the corresponding rate in noble-metals is estimated as [183 $\gamma_{e} \sim 10^{-2} \mathrm{eV}$. If one assumes the bulk value for $\mu\left(2 \mu^{2} / m \sim 1 \mathrm{eV}\right.$ near the L-point [184]), then $\gamma_{h}^{s}$ exceeds $\gamma_{e}$ for $R<2.5 \mathrm{~nm}$. In fact, one would expect that, in nanoparticles, $\mu$ is larger than in the bulk due to the localization of the conduction electron wave-functions 103 .

\section{Surface Plasmon Nonlinear Optical dynam- ics}

In this section, we discuss the effect of the SP-mediated interband scattering on the ultrafast optical dynamics in noble metal nanoparticles. We are interested in the situation when the hot electron distribution has already thermalized and the electron gas is cooling to the lattice (stage III). In this case the transient response of a nanoparticle can be described by the time-dependent absorption coefficient $\alpha(\omega, t)$, given by Eq. (12) with time-dependent temperature. In noble-metal particles, the temperature dependence of $\alpha$ originates from two different sources. First is the phonon-induced correction to $\gamma_{s}$, which is proportional to the lattice temperature $T_{l}(t)$. As mentioned in section 3, for small nanoparticles this effect is relatively weak. Note that, as discussed e.g. in Ref. [137], the contribution to $\gamma_{s}$ coming from the $e-e$ interaction 1125, 129, 130, 127, 139, 140, which depends on the electron temperature, plays a minor role when the interband transitions are resonantly excited. Second, near the onset of the interband transitions, $\Delta$, the absorption coefficient depends on the electron temperature $T(t)$ via the interband dielectric function $\epsilon_{d}(\omega)$ [see Eqs. (12) and (13)]. In fact, in copper or gold nanoparticles, $\omega_{s}$ can be tuned close to $\Delta$, so the SP damping by interband $e^{-}$ $h$ excitations leads to an additional broadening of the absorption peak 103. In this case, it is the temperature dependence of $\epsilon_{d}(\omega)$ that dominates the pump-probe dynamics. Below we show that, near the SP resonance, both the

temperature and frequency dependence of $\epsilon_{d}(\omega)=1+4 \pi \chi_{d}(\omega)$ are strongly affected by the SP-mediated interband scattering.

We start with the RPA expression for the interband susceptibility 43, 


$$
\begin{aligned}
\chi_{d}(i \omega)=\tilde{\chi}_{d}(i \omega)+\tilde{\chi}_{d}(-i \omega), & \\
\tilde{\chi}_{d}(i \omega) & =-\sum_{\alpha} \frac{e^{2} \mu^{2}}{m^{2}\left(E_{\alpha}^{c d}\right)^{2}} \frac{1}{\beta} \sum_{i \omega^{\prime}} G_{\alpha}^{d}\left(i \omega^{\prime}\right) G_{\alpha}^{c}\left(i \omega^{\prime}+i \omega\right),
\end{aligned}
$$

where $G_{\alpha}^{d}\left(i \omega^{\prime}\right)$ is the Green function of a $d$-electron. With the $d$-band fully occupied, the only allowed SP-mediated interband scattering is that of the $d$-hole. We assume here, for simplicity, a dispersionless $d$-band with energy $E^{d}$. Substituting $G_{\alpha}^{d}\left(i \omega^{\prime}\right)=\left[i \omega^{\prime}-E^{d}+E_{F}-\Sigma_{\alpha}^{d}\left(i \omega^{\prime}\right)\right]^{-1}$, with $\Sigma_{\alpha}^{d}(i \omega)$ given by Eq. (92), and performing the frequency summation, we obtain

$$
\tilde{\chi}_{d}(\omega)=\frac{e^{2} \mu^{2}}{m^{2}} \int \frac{d E^{c} g\left(E^{c}\right)}{\left(E^{c d}\right)^{2}} \frac{f\left(E^{c}\right)-1}{\omega-E^{c d}+i \gamma_{h}^{s}\left(\omega, E^{c}\right)},
$$

where $g\left(E^{c}\right)$ is the density of states of conduction electrons. The scattering rate of a $d$-hole, $\gamma_{h}^{s}\left(\omega, E^{c}\right)=\operatorname{Im} \Sigma^{d}\left(E^{c}-\omega\right)$, is obtained from Eq. (93) with $E_{d}=E^{c}-\omega:$

$$
\gamma_{h}^{s}\left(\omega, E^{c}\right)=-\frac{9 e^{2} \mu^{2}}{m^{2}\left(E^{c d}\right)^{2} R^{3}} f\left(E^{c}\right) \operatorname{Im} \frac{1}{\epsilon_{s}(\omega)},
$$

where $N(\omega)$ is negligible for frequencies $\omega \sim \omega_{s} \gg k_{B} T$ 152. The rate $\gamma_{h}^{s}\left(\omega, E^{c}\right)$ exhibits a sharp peak as a function of the frequency of the probe optical field. The reason for this is that the scattering rate of a $d$-hole with energy $E$ depends explicitly on the difference between the final and initial states, $E^{c}-E$, as discussed above; therefore, for a $d$-hole with energy $E=E^{c}-\omega$, the dependence on the final state energy, $E^{c}$, cancels out in $\epsilon_{s}\left(E^{c}-E\right)$ [see Eq. (93)]. In other words, the optically-excited $d$-hole scatters resonantly into the conduction band as $\omega$ approaches $\omega_{s}$. It is important to note that $\gamma_{h}^{s}\left(\omega, E^{c}\right)$ is, in fact, proportional to the absorption coefficient $\alpha(\omega)$ [see Eq. (12)]. Therefore, $\alpha$ and $\gamma_{h}^{s}$ should be calculated self-consistently from Eqs. (12), (13), (95), and (96).

It should be emphasized that the effect of $\gamma_{h}^{s}$ on $\epsilon_{d}^{\prime \prime}(\omega)$ increases with temperature. Indeed, it can be seen from Eq. (96) that the value of $\gamma_{h}^{s}$ is appreciable only if $E^{c}-E_{F}>k_{B} T$. Since the main contribution to $\tilde{\chi}_{d}^{\prime \prime}(\omega)$ comes from energies $E^{c} \sim \omega-\Delta+E_{F}$, the effect of $d$-hole scattering on the absorption becomes important only for elevated electron temperatures: $k_{B} T>\omega_{s}-\Delta$. As a result, near the SP resonance, the time evolution of the differential absorption, which is governed by the temperature dependence of $\alpha$, becomes strongly size-dependent. 
The numerical results discussed below are taken from Refs. 152, 153. In the experiment of Bigot et. al. [126], the pump-probe measurements were performed on $R \simeq 2.5 \mathrm{~nm}$ copper nanoparticles. The SP frequency, $\omega_{s} \simeq 2.22$ $\mathrm{eV}$, was slightly above the onset of the interband transitions, $\Delta \simeq 2.18 \mathrm{eV}$. In order to describe the time- evolution of the differential absorption spectra, one first needs to determine the time-dependence of the electron temperature, $T(t)$, due to the relaxation of the electron gas to the lattice. For this, a simple two-temperature model is employed, defined by heat equations for $T(t)$ and the lattice temperature $T_{l}(t)$ :

$$
\begin{aligned}
C(T) \frac{\partial T}{\partial t} & =-G\left(T-T_{l}\right), \\
C_{l} \frac{\partial T_{l}}{\partial t} & =G\left(T-T_{l}\right),
\end{aligned}
$$

where $C(T)=\gamma T$ and $C_{l}$ are the electron and lattice heat capacities, respectively, and $G$ is the $e-p$ coupling $[141$, 142, 143. The parameter values used in Refs. [152, 153 were $G=3.5 \times 10^{16} \mathrm{Wm}^{-3} \mathrm{~K}^{-1}, \gamma=70 \mathrm{Jm}^{-3} \mathrm{~K}^{-2}$, and $C_{l}=3.5 \mathrm{Jm}^{-3} \mathrm{~K}^{-1}$, and the initial condition was taken as $T_{0}=1000$ $\mathrm{K}$. The time-dependent absorption coefficient $\alpha(\omega, t)$ was calculated selfconsistently; the differential transmission is proportional to $\alpha_{r}(\omega)-\alpha(\omega, t)$, where $\alpha_{r}(\omega)$ was calculated at the room temperature.

In Fig. 13, the calculated nonlinear absorption spectra are shown for various nanoparticle sizes. Fig. 13(a) shows the spectra at several time delays for $R=5.0 \mathrm{~nm}$; for this size, the $\mathrm{SP}$-mediated $d$-hole scattering has no effect. With decreasing nanoparticle size, the linear absorption spectra are not significantly altered, as can be seen in Figs. 13(b) and (c). However, the change in the nonlinear absorption spectra becomes pronounced at short time delays corresponding to higher temperatures [see Figs. 13(b) and (c)]. This effect is more clearly seen in the differential transmission spectra, shown in Fig. 14, which undergo a qualitative transformation with decreasing size.

Note that it is necessary to include the intraband $e-e$ scattering in order to reproduce the differential transmission lineshape observed in the experiment [126]. For optically excited electron energy close to $E_{F}$, this can be achieved by adding the $e-e$ scattering rate [目] $\gamma_{e}\left(E^{c}\right) \propto\left[1-f\left(E^{c}\right)\right]\left[\left(E^{c}-E_{F}\right)^{2}+\right.$ $\left.\left(\pi k_{B} T\right)^{2}\right]$ to $\gamma_{h}^{s}$ in Eq. (95). The difference in $\gamma_{e}\left(E^{c}\right)$ for $E^{c}$ below and above $E_{F}$ leads to a lineshape similar to that expected from the combination of red-shift and broadening [see Fig. 14(a)].

In Figs. 14(b) and (c) the differential transmission spectra are shown 
with decreasing nanoparticle size. For $R=2.5 \mathrm{~nm}$, the apparent red-shift is reduced [see Fig. 14(b)]. This change can be explained as follows. Since here $\omega_{s} \sim \Delta$, the SP is damped by the interband excitations. This broadens the spectra for $\omega>\omega_{s}$, so that the absorption peak is asymmetric. The $d-$ hole scattering with the SP enhances the damping; since the $\omega$-dependence of $\gamma_{h}^{s}$ follows that of $\alpha$, this effect is larger above the resonance. On the other hand, the efficiency of the scattering increases with temperature, as discussed above. Therefore, for short time delays, the relative increase in the absorption is larger for $\omega>\omega_{s}$. With decreasing size, the strength of this effect increases further, leading to an apparent blue-shift [see Fig. [14(c)]. Such a strong change in the absorption dynamics originates from the $R^{-3}$ dependence of the $d$-hole scattering rate; reducing the size by the factor of two results in an enhancement of $\gamma_{h}^{s}$ by an order of magnitude.

In Fig. 15, the time evolution of the differential transmission are shown for several frequencies close to $\omega_{s}$. It can be seen that the relaxation is slowest at the SP resonance; this characterizes the robustness of the collective mode, which determines the peak position, versus the single-particle excitations, which determine the resonance width. For larger sizes, at which $\gamma_{h}^{s}$ is small, the change in the differential transmission decay rate with frequency is smoother above the resonance [see Fig. 15(a)]. This stems from the asymmetric lineshape of the absorption peak, mentioned above: the absorption is larger for $\omega>\omega_{s}$, so that its relative change with temperature is weaker. For smaller nanoparticle size, the decay rates become similar above and below $\omega_{s}$ [see Fig. 15(b)]. This change in the frequency dependence is related to the stronger SP damping for $\omega>\omega_{s}$ due to the $d$-hole scattering, as discussed above. Since this additional damping is reduced with decreasing temperature, the relaxation is faster above the resonance. This rather "nonlinear" relation between the time-evolution of the pump-probe signal and that of the temperature becomes even stronger for smaller sizes [see Fig. 15(c)]. In this case, the frequency dependence of the differential transmission decay below and above $\omega_{s}$ is reversed. Note that a frequency dependence consistent with our calculations presented in Fig. 15(b) was, in fact, observed in the experiment of Ref. [126], shown in Fig. 15(d). 


\section{Conclusions}

In this article we presented an overview of ultrafast nonlinear optics experiments that probe the dynamics of modulation-doped quantum wells and metal nanoparticles. We also discussed some examples where the ultrafast dynamics of such confined Fermi seas cannot be described in terms of the usual dephasing and relaxation time approximations. These examples show the important role of dynamical and non-equilibrium many-body correlations during femtosecond time scales.

More specifically, we discussed a recent theory for the ultrafast nonlinear optical response of the FES. We focussed on coherent effects, which dominate the pump-probe spectra during negative time delays and off-resonant excitation conditions. We demonstrated that the dynamical FS response leads to qualitatively different coherent dynamics of the FES as compared to the Hartree-Fock approximation. In the latter case, the time evolution of the resonance bleaching is governed by the dephasing time. In contrast, in the former case, polarization interference and $e-h$ correlation effects dominate. This results in an initial fast FES dynamics, with a response time determined by the characteristic inverse Coulomb energy $E_{M}$ as well as an apparent resonance enhancement during negative time delays, followed by a long-time decay determined by the dephasing time. Such dynamical features should be observable in ultrafast PP experiments. Using a simple model, we showed that the different dynamics of the FES and Hartree-Fock treatment can be attributed to the non-Lorentzian broadening of the HFA bound state due to its interactions with the gapless FS excitations, a process which is, of course, beyond the dephasing time approximation. In addition, we showed that the pump excitation directly affects the strength of the $e-h$ scattering processes, which changes the frequency dependence of the resonance. The latter can be thought of as an excitation-induced dephasing effect that leads to a transient enhancement of the FES. These results indicate that ultrafast spectroscopy provides a powerful tool to study the role of correlations in the nonlinear response of a Fermi liquid during time scales shorter than the dephasing times. The dynamical features discussed above can also be used as an experimental signal to probe the crossover from FES to exciton bound state (exciton Mott transition) as a function of the FS density.

The above $e-h$ correlation effects and the fundamental differences in the dynamics between an exciton state and a FES many-body resonance can be best observed experimentally in asymmetric modulation-doped quantum 
wells in the case where the lowest occupied subband is in close proximity to the second unoccupied subband and is coupled strongly to it via intersubband $e-h$ scattering. In such a system there is a strong redistribution of the oscillator strength between the FES and exciton peaks which is caused by the different dynamics of the FES and exciton components of the hybrid as well as by their coupling due to the $e-h$ correlations. This originates from the dynamical Fermi sea response and leads to a strong transient changes in the PP spectra.

We also discussed the role of size-dependent correlations in the electron relaxation in small metal particles. We identified a new mechanism of quasiparticle scattering, mediated by collective surface excitations, which originates from the surface-induced dynamical screening of the $e-e$ interactions. The behavior of the corresponding scattering rates with varying energy and temperature differs substantially from that in the bulk metal. We showed that the conduction electron scattering rate increases with energy, in sharp contrast to the bulk behavior, which could be observed in two-photon photoemission measurements. We also found that in noble metal particles, the resonant energy dependence of the $d$-hole scattering rate affects strongly the differential absorption.

An important aspect of the $\mathrm{SP}$-mediated scattering is its strong dependence on size. Our estimates show that it becomes comparable to the usual Fermi-liquid scattering in nanometer-sized particles. This size regime is, in fact, intermediate between "classical" particles with sizes larger than 10 $\mathrm{nm}$, where the bulk-like behavior dominates, and very small clusters with only dozens of atoms, where the metallic properties are completely lost. Although the static properties of nanometer-sized particles are also sizedependent, the deviations from their bulk values do not change the qualitative features of the electron dynamics. In contrast, the size-dependent many-body effects discussed here do affect the dynamics in a significant way during time scales comparable to the relaxation times. The SP-mediated interband scattering reveals itself in the size-dependence of the transient pump-probe spectra. In particular, as the nanoparticle size decreases, the calculated time-resolved differential absorption lineshape shows a transition from an apparent redshift to a blueshift. This transition, absent in the RPA, comes from the correlations between collective surface and single-particle excitations. At the same time, near the SP resonance, these correlation leads to significant size-dependent changes in the frequency dependence of the relaxation time of the pump-probe signal. These results indicate the need for 
a systematic experimental studies of the size-dependence of the transient nonlinear optical response, as we approach the transition from boundaryconstrained nanoparticles to molecular clusters. We expect that in the coming years ultrafast nonlinear optical spectroscopy will provide new insight into many-body effects in strongly correlated, and magnetic Fermi sea systems [185, 186, 187, 188, 189, 190].

\section{Acknowledgements}

This work was supported by the NSF grant ECS-9703453 and by HITACHI Ltd. Many of the calculations discussed in this review were performed by N. Primozich. It is a pleasure to acknowledge numerous fruitful discussions with D. S. Chemla and J.-Y. Bigot.

\section{A APPENDIX A}

In this appendix we briefly outline the formalism of Ref. [154. The pumpprobe signal is determined by the polarization,

$$
P(t)=\mu e^{-i \omega_{p} t}\langle\Psi(t)|U| \Psi(t)\rangle
$$

where the state $|\Psi(t)\rangle$ satisfies the time-dependent Schrödinger equation,

$$
\left[i \frac{\partial}{\partial t}-H_{t o t}(t)\right]|\Psi(t)\rangle=\left[i \frac{\partial}{\partial t}-H-H_{p}(t)-H_{s}(t)\right]|\Psi(t)\rangle=0,
$$

with the Hamiltonians $H$ and $H_{p, s}(t)$, given by Eqs. (16)-(17). The Hilbert space of the bare semiconductor, i.e. in the absence of optical fields, consists of disconnected subspaces $\zeta\left\{\nu_{e h}\right\}$ which are labeled by the number of (interband) $e-h$ pairs, $\nu_{e h}$. The corresponding bare Hamiltonian, $H$, conserves the number of $e-h$ pairs in each band separately and in the $\zeta\left\{\nu_{e h}\right\}$-basis has a block-diagonal form. The Hamiltonians $H_{p, s}(t)$ couple the different subspaces $\zeta\left\{\nu_{e h}\right\}$ by causing interband transitions.

In the description of $\mathrm{PP}$ experiments, we are interested only in the polarization component propagating along the probe direction $\mathbf{k}_{s}$. For a weak probe, the nonlinear signal then arises from the linear response of the system in the presence of the pump, described by the time-dependent Hamiltonian $H+H_{p}(t)$, to the probe-induced perturbation $H_{s}(t)$. Within $\chi^{(3)}$, the above 
is true even for comparable pump and probe amplitudes. However, since, in contrast to $H$, the Hamiltonian $H+H_{p}(t)$ does not conserve the number of carriers in each band separately, the calculation of the linear response function is not practical. Therefore, we seek to replace $H+H_{p}(t)$ by an effective Hamiltonian $\tilde{H}(t)$ that does conserve the number of $e-h$ pairs in each of its Hilbert subspaces (i.e., is "block-diagonal"). As derived in Ref. [154], this can be accomplished in any given order in the pump field $\mathcal{E}_{p}(t)$ and for any pulse duration by using a time-dependent Schrieffer-Wolff /Van Vleck canonical transformation [158, 160]. Here it is sufficient to "block-diagonalize" the Hamiltonian $H+H_{p}(t)$ up to the second order in $\mathcal{E}_{p}(t)$. The transformation that achieves this has the form $e^{-\hat{T}_{2}} e^{-\hat{T}_{1}}\left[H+H_{p}(t)\right] e^{\hat{T}_{1}} e^{\hat{T}_{2}}$, where the antiHermitian operators $\hat{T}_{1}(t)$ and $\hat{T}_{2}(t)$ create/annihilate one and two $e-h$ pairs, respectively.

We proceed with the first step and eliminate the single-pair pump-induced transitions in the time-dependent Schrödinger equation of the pump/baresemiconductor system,

$$
\left[i \frac{\partial}{\partial t}-H-H_{p}(t)\right]|\Psi(t)\rangle=0 .
$$

This is achieved by substituting $|\Psi(t)\rangle=e^{\hat{T}_{1}(t)}|\chi(t)\rangle$ and acting with the operator $e^{-\hat{T}_{1}(t)}$ on the lhs of Eq. (100),

$$
e^{-\hat{T}_{1}(t)}\left[i \frac{\partial}{\partial t}-H\right] e^{\hat{T}_{1}(t)}|\chi(t)\rangle=e^{-\hat{T}_{1}(t)}\left[H_{p}(t)\right] e^{\hat{T}_{1}(t)}|\chi(t)\rangle .
$$

The anti-Hermitian operator $\hat{T}_{1}(t)$ has a decomposition

$$
\hat{T}_{1}(t)=\hat{\mathcal{P}}(t) e^{-i \mathbf{k}_{p} \cdot \mathbf{r}}-\hat{\mathcal{P}}^{\dagger}(t) e^{i \mathbf{k}_{p} \cdot \mathbf{r}},
$$

where $\hat{\mathcal{P}}^{\dagger}(t)$ and $\hat{\mathcal{P}}(t)$ create and annihilate single $e-h$ pairs, respectively. The effective Hamiltonian can be found from the condition that the terms describing single-pair interband transitions cancel each other in Eq. (101). In Ref. [154], it was shown that the multiple commutators of $\hat{\mathcal{P}}(t)$ with its time derivatives can be eliminated from Eq. (101) to all orders. By expanding Eq. (101) and neglecting third or higher order terms in $\hat{\mathcal{P}}$, we obtain the following equation:

$$
i \frac{\partial \hat{\mathcal{P}}^{\dagger}(t)}{\partial t}=\left[H, \hat{\mathcal{P}}^{\dagger}(t)\right]+\mu \mathcal{E}_{p}(t) U^{\dagger}
$$


with initial condition $\hat{\mathcal{P}}^{\dagger}(-\infty)=0$. The formal solution is

$$
\hat{\mathcal{P}}^{\dagger}(t)=i \mu \int_{-\infty}^{t} d t^{\prime} \mathcal{E}_{p}\left(t^{\prime}\right) e^{-i H\left(t-t^{\prime}\right)} U^{\dagger} e^{i H\left(t-t^{\prime}\right)} .
$$

Note that, since the Hamiltonian $H$ conserves the number of $e-h$ pairs and the optical transition operator $U^{\dagger}$ creates a single $e-h$ pair, $\hat{\mathcal{P}}^{\dagger}(t)$ also creates a single $e^{-h}$ pair. Furthermore, since both $H$ and $U^{\dagger}$ conserve momentum, so does $\hat{\mathcal{P}}^{\dagger}(t)$. Eq. (101) then takes the form

$$
\left[i \frac{\partial}{\partial t}-\tilde{H}(t)\right]|\chi(t)\rangle=+\frac{\mu}{2}\left[\mathcal{E}_{p}(t) e^{2 i \mathbf{k}_{p} \mathbf{r}}\left[U^{\dagger}, \hat{\mathcal{P}}^{\dagger}(t)\right]+\text { H.c. }\right]|\chi(t)\rangle,
$$

where

$$
\tilde{H}(t)=H+\frac{\mu}{2}\left(\mathcal{E}_{p}(t)\left[\hat{\mathcal{P}}(t), U^{\dagger}\right]+\text { H.c. }\right)
$$

is the sought time-dependent effective Hamiltonian that conserves the number of $e-h$ pairs and $\hat{\mathcal{P}}^{\dagger}(t)$ is given by Eq. (103). Note that, since $\hat{\mathcal{P}}^{\dagger}(t)$ is linear in the pump field $\mathcal{E}_{p}$, the pump-induced term in $\tilde{H}(t)$ [second term in Eq. (106)] is quadratic $\left(\propto \mathcal{E}_{p} \mathcal{E}_{p}^{*}\right)$. The rhs of Eq. (105) describes the pumpinduced two-pair transitions. These can be eliminated as well by performing a second canonical transformation, $|\chi(t)\rangle=e^{\hat{T}_{2}(t)}|\Phi(t)\rangle$. Following the same procedure, we use the anti-Hermiticity of $\hat{T}_{2}(t)$ to decompose it as

$$
\hat{T}_{2}(t)=\hat{\mathcal{P}}_{2}(t) e^{-2 i \mathbf{k}_{p} \cdot \mathbf{r}}-\mathcal{P}_{2}^{\dagger}(t) e^{2 i \mathbf{k}_{p} \cdot \mathbf{r}},
$$

where $\mathcal{P}_{2}^{\dagger}(t)$ and $\hat{\mathcal{P}}_{2}(t)$ create and annihilate two $e-h$ pairs, respectively. Substituting the above expression for $|\chi(t)\rangle$ into Eq. (105) and requiring that all two-pair transitions cancel out, we obtain the following equation for $\mathcal{P}_{2}^{\dagger}(t)$,

$$
i \frac{\partial \mathcal{P}_{2}^{\dagger}(t)}{\partial t}=\left[\tilde{H}(t), \mathcal{P}_{2}^{\dagger}(t)\right]+\frac{\mu}{2} \mathcal{E}_{p}(t)\left[\hat{\mathcal{P}}^{\dagger}(t), U^{\dagger}\right]
$$

Note that $\hat{\mathcal{P}}_{2}^{\dagger}(t)$ only affects the PP polarization via higher order $\left(\mathcal{E}_{p}^{4}\right)$ corrections, which are neglected here. However, it does determine the four-wavemixing (FWM) polarization (see below).

To obtain the condition of validity of this approach, it is useful to write down a formal solution (104) of Eq. (103) in the basis of the N-hole manybody eigenstates, $|\alpha N\rangle$, with energies $E_{\alpha N}$, of the Hamiltonian $H$. Here the index $\alpha$ labels all the other quantum numbers, so that $N=0$ corresponds to 
the semiconductor ground state $|0\rangle,|\alpha 1\rangle$ denotes the one-pair states (exciton eigenstates in the undoped case, with $\alpha$ labeling both bound and scattering states), $|\alpha 2\rangle$ denotes the two-pair (biexciton in the undoped case) eigenstates, etc. In this basis, the solution of Eq. (103) can be written as

$$
\frac{\left\langle\beta N+1\left|\hat{\mathcal{P}}^{\dagger}(t)\right| \alpha N\right\rangle}{\left\langle\beta N+1\left|U^{\dagger}\right| \alpha N\right\rangle}=-i \mu \int_{-\infty}^{t} d t^{\prime} \mathcal{E}_{p}\left(t^{\prime}\right) e^{-i\left(t-t^{\prime}\right)\left(\Omega+\Delta E_{\alpha \beta}^{N}\right)} e^{-\Gamma\left(t-t^{\prime}\right)},
$$

where we separated out the detuning $\Omega$ and denoted $\Delta E_{\alpha \beta}^{N}=E_{\beta N+1}-E_{\alpha N}$. It can be seen that for resonant excitation (small $\Omega$ ) the rhs of Eq. (109) is of the order of $\mu \mathcal{E}_{p} t_{p}$. Thus, for short pulses, this parameter justifies the expansion in terms of the optical fields. For off-resonant excitation, this expansion is valid for any pulse duration provided that $\mu \mathcal{E}_{p} / \Omega<1$. Similar conditions can be obtained for the two-pair transition described by $\hat{\mathcal{P}}_{2}$.

The nonlinear polarization Eq. (98) can now be expressed in terms of the linear response to the probe field:

$$
P(t)=\mu e^{-i \omega_{p} t}\langle\Psi(t)|U| \Psi(t)\rangle=\mu e^{-i \omega_{p} t}\left\langle\Phi(t)\left|U_{T}(t)\right| \Phi(t)\right\rangle,
$$

where, in the first order in $\mathcal{E}_{s}(t)$, the state $|\Phi(t)\rangle$ is given by

$$
|\Phi(t)\rangle=\left|\Phi_{0}(t)\right\rangle-i \mu \int_{-\infty}^{t} d t^{\prime} \mathcal{K}\left(t, t^{\prime}\right)\left[\mathcal{E}_{s}\left(t^{\prime}\right) e^{i \mathbf{k}_{s} \mathbf{r}+i \omega_{p} \tau} U_{T}^{\dagger}\left(t^{\prime}\right)+\text { H.c. }\right]\left|\Phi_{0}\left(t^{\prime}\right)\right\rangle .
$$

Here $\mathcal{K}\left(t, t^{\prime}\right)$ is the time-evolution operator satisfying

$$
i \frac{\partial}{\partial t} \mathcal{K}\left(t, t^{\prime}\right)=\tilde{H}(t) \mathcal{K}\left(t, t^{\prime}\right)
$$

and $U_{T}^{\dagger}(t)=e^{-\hat{T}_{2}(t)} e^{-\hat{T}_{1}(t)} U^{\dagger} e^{\hat{T}_{1}(t)} e^{\hat{T}_{2}(t)}$ is the (transformed) optical transition operator. In Eq. (111), $\left|\Phi_{0}(t)\right\rangle=\mathcal{K}(t,-\infty)|0\rangle$ is the time-evolved ground state $|0\rangle$; since $\tilde{H}(t)$ conserves the number of $e-h$ pairs, $\left|\Phi_{0}(t)\right\rangle$ contains no interband $e-h$ pairs (in undoped semiconductors, it coincides with the ground state, $\left.\left|\Phi_{0}(t)\right\rangle=|0\rangle\right)$. ¿From Eqs. (111) and (110), the polarization $P(t)$ takes the form

$$
\begin{aligned}
P(t)=i \mu^{2} e^{-i \omega_{p} t} & \int_{-\infty}^{t} d t^{\prime}\left[\langle \Phi _ { 0 } ( t ) | U _ { T } ( t ) \mathcal { K } ( t , t ^ { \prime } ) \left[\mathcal{E}_{s}\left(t^{\prime}\right) e^{i \mathbf{k}_{s} \cdot \mathbf{r}+i \omega_{p} \tau} U_{T}^{\dagger}\left(t^{\prime}\right)\right.\right. \\
& \left.+\mathcal{E}_{s}^{*}\left(t^{\prime}\right) e^{-i \mathbf{k}_{s} \cdot \mathbf{r}-i \omega_{p} \tau} U_{T}\left(t^{\prime}\right)\right]\left|\Phi_{0}\left(t^{\prime}\right)\right\rangle \\
& -\left\langle\Phi_{0}\left(t^{\prime}\right)\right|\left[\mathcal{E}_{s}\left(t^{\prime}\right) e^{i \mathbf{k}_{s} \cdot \mathbf{r}+i \omega_{p} \tau} U_{T}^{\dagger}\left(t^{\prime}\right)\right. \\
& \left.\left.+\mathcal{E}_{s}^{*}\left(t^{\prime}\right) e^{-i \mathbf{k}_{s} \cdot \mathbf{r}-i \omega_{p} \tau} U_{T}\left(t^{\prime}\right)\right] \mathcal{K}\left(t^{\prime}, t\right) U_{T}(t)\left|\Phi_{0}(t)\right\rangle\right]
\end{aligned}
$$


The above expression for the total polarization contains contributions propagating in various directions. To obtain the polarization propagating in a specific direction, one has to expand the effective-transition operator $U_{T}(t)$ in terms of $\hat{T}_{1}$ and $\hat{T}_{2}$. Using Eqs. (102) and (107) and keeping only terms contributing to PP and FWM polarizations, we obtain [154

$$
U_{T}^{\dagger}(t)=U_{1}^{\dagger}(t)+U_{2}^{\dagger}(t) e^{i \mathbf{k}_{p} \mathbf{r}}+U_{F W M}(t) e^{-2 i \mathbf{k}_{p} \mathbf{r}}+\cdots,
$$

where (to lowest order in the pump field)

$$
\begin{aligned}
U_{1}^{\dagger}(t) & =U^{\dagger}+\frac{1}{2}\left[\hat{\mathcal{P}}(t),\left[U^{\dagger}, \hat{\mathcal{P}}^{\dagger}(t)\right]\right]+\frac{1}{2}\left[\hat{\mathcal{P}}^{\dagger}(t),\left[U^{\dagger}, \hat{\mathcal{P}}(t)\right]\right], \\
U_{2}^{\dagger}(t) & =\left[\hat{\mathcal{P}}^{\dagger}(t), U^{\dagger}\right], \\
U_{F W M}^{\dagger}(t) & =\frac{1}{2}\left[\left[U, \hat{\mathcal{P}}^{\dagger}(t)\right], \hat{\mathcal{P}}^{\dagger}(t)\right]-\left[U, \hat{\mathcal{P}}_{2}^{\dagger}(t)\right] .
\end{aligned}
$$

Here operators $U_{1}^{\dagger}(t) \equiv \tilde{U}(t)$ and $U_{F W M}^{\dagger}(t)$ create one $e-h$ pair, while $U_{2}^{\dagger}(t)$ creates two $e-h$ pairs (note that $U_{F W M}$ in Eq. (115) annihilates an $e-h$ pair).

\section{Pump-probe polarization}

In order to extract the PP polarization from Eq. (113), one should retain only terms that are proportional to $e^{i \mathbf{k}_{s} \cdot \mathbf{r}}$. Substituting Eqs. (114) into Eq. (113), we obtain $P_{\mathbf{k}_{s}}(t)=e^{i \mathbf{k}_{s} \cdot \mathbf{r}-i \omega_{p}(t-\tau)}\left[\tilde{P}^{(1)}(t)+\tilde{P}^{(2)}(t)\right]$, where

$$
\tilde{P}^{(1)}(t)=i \mu^{2} \int_{-\infty}^{t} d t^{\prime} \mathcal{E}_{s}\left(t^{\prime}\right)\left\langle\Phi_{0}(t)\left|\tilde{U}(t) \mathcal{K}\left(t, t^{\prime}\right) \tilde{U}^{\dagger}\left(t^{\prime}\right)\right| \Phi_{0}\left(t^{\prime}\right)\right\rangle
$$

and

$$
\tilde{P}^{(2)}(t)=i \mu^{2} \int_{-\infty}^{t} d t^{\prime} \mathcal{E}_{s}\left(t^{\prime}\right)\left\langle\Phi_{0}(t)\left|U_{2}(t) \mathcal{K}\left(t, t^{\prime}\right) U_{2}^{\dagger}\left(t^{\prime}\right)\right| \Phi_{0}\left(t^{\prime}\right)\right\rangle
$$

Note that the above formulae apply to both undoped and doped semiconductors.

Eqs. (116)-(117) express the nonlinear PP polarization in terms of the linear response to the probe field of a system described by the time-dependent effective Hamiltonian (106). Such a form for the nonlinear response allows one to distinguish between two physically distinct contributions to the optical nonlinearities. Assuming that a short probe pulse arrives at $t=\tau$, consider 
the first term, Eq. (116), which gives the single-pair (exciton for undoped case) contribution to the PP polarization. At negative time delays, $\tau<0$, the probe excites an $e-h$ pair, described by state $\tilde{U}^{\dagger}(\tau)\left|\Phi_{0}(\tau)\right\rangle \simeq U^{\dagger}|0\rangle$; since the probe arrives before the pump, the effective transition operator coincides with the "bare" one [see Eqs. (115) and (104)]. The first contribution to the optical nonlinearities comes from the effective Hamiltonian, $\tilde{H}(t)$, governing the propagation of that interacting $e-h$ pair in the interval $(\tau, t)$ via the time-evolution operator $\mathcal{K}(t, \tau)$. Note that since the pump pulse arrives at $t=0$, for $|\tau| \gg \Gamma^{-1}$, the negative time-delay signal vanishes. At $t>0$, the $e-h$ pair (exciton in the undoped case) "feels" the effect of the pump via mainly the transient bandgap shift, leading, e.g., to ac-Stark effect, and the change in the band dispersions (increase in effective mass/density of states), leading to enhanced $e-h$ scattering (exciton binding energy in the undoped case). Note that $\tilde{H}(t)$ also contains a contribution coming from the interactions between probe- and pump-excited $e-h$ pairs, which are however perturbative in the doped case for short pulses or off-resonant excitation and lead to subdominant corrections. Importantly, the response of the system to the optically-induced corrections in $\tilde{H}(t)$ takes into account all orders in the pump field, which is necessary for the adequate description, e.g., of the ac-Stark effect and the pump-induced changes in the $e-h$ correlations. Indeed, although the pump-induced term in Eq. (106) is quadratic in $\mathcal{E}_{p}$, the time-evolution of the interacting $e-h$ pair is described without expanding $\mathcal{K}(t, \tau)$ in the pump field. On the other hand, the third-order polarization $\left(\chi^{(3)}\right)$ can be obtained by expanding $\mathcal{K}(t, \tau)$ to the lowest order. The second contribution to the optical nonlinearities comes from the matrix element of the final state, $\left\langle\Phi_{0}(t)\right| \tilde{U}(t)$ in Eq. (116). The latter, given by Eq. (115), contains the lowest order (quadratic) pump-induced terms which describe the Pauli blocking, pair-pair, and pair-FS interaction effects (exciton-exciton interactions in the undoped case [154]). Note that the matrix element of the initial state contributes for positive time delays, i.e., if the probe arrives after the pump pulse. In this case, however, the pump-induced term in the effective Hamiltonian (106) vanishes (since it lasts only for the duration of the pulse) so that for positive $\tau>t_{p}$ the PP signal is determined by the matrix elements rather than by $\tilde{H}(t)$. If the probe arrives during the interaction of the system with the pump pulse $\left(\tau \sim t_{p}\right)$, both the effective Hamiltonian and the matrix elements contribute to the polarization. In this case, there is also a biexcitonic contribution [given by Eq. (117)] coming from a simultaneous excitation of two $e-h$ pairs by the pump and the probe. However, such a 
biexciton state does not contribute to negative $(\tau<0)$ time delays. As can be seen from the above discussion, our theory separates out a number of contributions that play a different role for different time delays and excitation conditions.

\section{FWM polarization}

By extracting from Eq. (113) all the terms propagating in the the FWM direction, $2 \mathbf{k}_{p}-\mathbf{k}_{s}$, we obtain [154] (for delta-function probe $\mathcal{E}_{s}(t)=\mathcal{E}_{s} \delta(t-$ $\tau))$

$$
\begin{aligned}
P_{F W M}(t)= & i e^{i\left(2 \mathbf{k}_{p}-\mathbf{k}_{s}\right) \cdot \mathbf{r}-i \omega_{p}(t+\tau)} \theta(t-\tau) \mu^{2} \mathcal{E}_{s}^{*} \\
& \times\left[\left\langle\Phi_{0}(t)\left|U \mathcal{K}(t, \tau) U_{F W M}^{\dagger}(\tau)\right| \Phi_{0}(\tau)\right\rangle-(t \leftrightarrow \tau)\right],
\end{aligned}
$$

where the FWM transition operator $U_{F W M}^{\dagger}(t)$ is given by Eq. (115). It is convenient to express $U_{F W M}^{\dagger}(t)$ in terms of the "irreducible" two-pair operator $W^{\dagger}(t)=\frac{1}{2} \hat{\mathcal{P}}^{\dagger 2}-\hat{\mathcal{P}}_{2}^{\dagger}$, satisfying

$$
i \frac{\partial W^{\dagger}(t)}{\partial t}=\left[\tilde{H}(t), W^{\dagger}(t)\right]+\mu \mathcal{E}_{p}(t) U^{\dagger} \hat{\mathcal{P}}^{\dagger}(t)
$$

In terms of $W^{\dagger}(t)$, the state $U_{F W M}^{\dagger}(t)\left|\Phi_{0}(t)\right\rangle$ in Eq. (118) can be presented as a sum of two- and one-pair contributions:

$$
U_{F W M}^{\dagger}(t)\left|\Phi_{0}(t)\right\rangle=U W^{\dagger}(t)\left|\Phi_{0}(t)\right\rangle-\hat{\mathcal{P}}^{\dagger}(t) U \hat{\mathcal{P}}^{\dagger}(t)\left|\Phi_{0}(t)\right\rangle .
$$

For undoped semiconductors, $\left|\Phi_{0}(t)\right\rangle$ represents the ground state $|0\rangle$ of $H$. The operator $W^{\dagger}(t)$, being quadratic in the pump field $\left(\propto \mathcal{E}_{p}^{2}\right)$, describes the simultaneous excitation of two interacting $e-h$ pairs by the pump pulse and includes the biexciton and exciton-exciton scattering effects. Introducing the amplidutes $\chi_{\mathbf{k}}$ and $\Phi_{\mathbf{k}}$ as

$$
U_{F W M}^{\dagger}(t)|0\rangle=\sum_{\mathbf{k}} \chi_{\mathbf{k}}(t) a_{\mathbf{k}}^{\dagger} b_{-\mathbf{k}}^{\dagger}|0\rangle,
$$

and

$$
\tilde{\mathcal{K}}\left(t, t^{\prime}\right) U_{F W M}^{\dagger}\left(t^{\prime}\right)|0\rangle=\sum_{\mathbf{k}} \Phi_{\mathbf{k}}\left(t, t^{\prime}\right) a_{\mathbf{k}}^{\dagger} b_{-\mathbf{k}}^{\dagger}|0\rangle
$$


where $\Phi_{\mathbf{k}}\left(t, t^{\prime}\right)$ satisfies

$$
i \partial_{t} \Phi_{\mathbf{k}}\left(t, t^{\prime}\right)=\sum_{\mathbf{q}}\left\langle 0\left|b_{-\mathbf{k}} a_{\mathbf{k}} \tilde{H}(t) a_{\mathbf{q}}^{\dagger} b_{-\mathbf{q}}^{\dagger}\right| 0\right\rangle \Phi_{\mathbf{q}}\left(t, t^{\prime}\right),
$$

with initial condition $\Phi_{\mathbf{k}}(t, t)=\chi_{\mathbf{k}}(t)$, one finally obtains for the FWM polarization

$$
P_{F W M}(t)=i e^{i\left(2 \mathbf{k}_{p}-\mathbf{k}_{s}\right) \cdot \mathbf{r}-i \omega_{p}(t+\tau)} \theta(t-\tau) \mu^{2} \mathcal{E}_{s}^{*} \sum_{\mathbf{k}}\left[\Phi_{\mathbf{k}}(t, \tau)-\Phi_{\mathbf{k}}(\tau, t)\right] .
$$

Note that the third order polarization [corresponding to $\chi^{(3)}$ ] is obtained by replacing $\tilde{H}$ with $H$.

\section{B APPENDIX B}

In this appendix we clarify our convention for the time delay $\tau$ and relate it to the most commonly used conventions in PP and FWM. In the generic experimental configuration two laser pulses $E_{1}(t) e^{i \mathbf{k}_{1} \cdot \mathbf{r}-i \omega\left(t-t_{1}\right)}$, and $E_{2}(t) e^{i \mathbf{k}_{2} \cdot \mathbf{r}-i \omega\left(t-t_{2}\right)}$, respectively centered at time $t=t_{1}$ and $t=t_{2}$ are incident on a sample. Let us define $\Delta t$ as,

$$
\Delta t=t_{1}-t_{2}
$$

and consider a FWM experiment where the signal is measured in the direction $2 \mathbf{k}_{2}-\mathbf{k}_{1}$. Then for a two-level-atom, the signal vanishes for $\Delta t<0$, while for $\Delta t>0$ its amplitude, which decays with time as $e^{-t / T_{2}}$, is determined by the Pauli blocking. In a system with Coulomb interactions (such as a semiconductor) a FWM signal is observed both for $\Delta t<0$ and $\Delta t>0$. The $\Delta t<0$ signal is entirely due to the Coulomb interaction.

In PP experiments, one usually chooses one pulse (the "pump") to have an amplitude $E_{p}$ much larger than that of the other pulse (the "probe"), $E_{s}$. As discussed in the text, a weak probe measures the linear response of the system (bare or dressed by the pump). If we choose that $E_{p}=E_{2}$, the pump induces coherent and incoherent populations when it arrives in the sample before the probe i.e. for $t_{2}<t_{1}$. This is usually defined as "positive" time delay $\tau=t_{2}-t_{1}>0$ in the PP literature. Note that $\tau=-\Delta t$, i.e., the "regular" sequence in PP experiments is the reverse of that of FWM

experiments. For $\tau<0$, the origin of the PP signal is that the probe creates 
a linear polarization in the sample which lasts for time $\sim T_{2}=\Gamma^{-1}$ and, consequently, is scattered by polarization excited by the pump field. The signal observed for $\tau<0$ is therefore due to coherent effects.

In FWM experiments, there is no restriction on the magnitude of the two incident fields $E_{2}$ and $E_{1}$, which are often chosen to have amplitudes of the same order. Note however that, at the $\chi^{(3)}$ level, the FWM and pump-probe polarizations are linear in the $E_{1}(t)$ field and thus the above linear response calculation applies even for comparable pump and probe amplitudes.

\section{APPENDIX C}

In this Appendix we present the explicit expressions for the renormalized transition matrix elements in the presence of the pump excitation. The direct transition matrix element is given by

$$
\begin{aligned}
M_{\mathbf{p}}(t) & =1-\left|\mathcal{P}_{e h}(\mathbf{p}, t)\right|^{2}+\left[\mathcal{P}_{\text {eh }}^{*}(\mathbf{p}, t) \sum_{k^{\prime}<k_{F}} \mathcal{P}_{e h}^{\mathrm{e}}\left(\mathbf{p k}^{\prime} ; \mathbf{k}^{\prime} ; t\right)+\text { H.c. }\right] \\
& -\frac{1}{2} \sum_{p^{\prime}>k_{F}} \mathcal{P}_{e h}^{*}\left(\mathbf{p}^{\prime}, t\right)\left[\mathcal{P}_{e h}^{\mathrm{e}}\left(\mathbf{p}^{\prime} \mathbf{p} ; \mathbf{p}^{\prime} ; t\right)-\mathcal{P}_{e h}^{\mathrm{h}}\left(\mathbf{p}^{\prime} \mathbf{p} ; \mathbf{p}^{\prime} ; t\right)\right. \\
& \left.-\mathcal{P}_{e h}^{\mathrm{h}}\left(\mathbf{p}^{\prime} \mathbf{p} ; \mathbf{p} ; t\right)+\mathcal{P}_{e h}^{\mathrm{e}}\left(\mathbf{p}^{\prime} \mathbf{p} ; \mathbf{p} ; t\right)\right] \\
& +\frac{1}{2} \sum_{p^{\prime}>k_{F}}\left[\mathcal{P}_{e h}(\mathbf{p}, t)+\mathcal{P}_{e h}\left(\mathbf{p}^{\prime}, t\right)\right] \\
& \times\left[\mathcal{P}_{e h}^{\mathrm{e}}\left(\mathbf{p p}^{\prime} ; \mathbf{p} ; t\right)+\mathcal{P}_{e h}^{\mathrm{h}}\left(\mathbf{p} \mathbf{p}^{\prime} ; \mathbf{p} ; t\right)\right]^{*} .
\end{aligned}
$$

The first term on the rhs of the above equation describes the phase space filling contribution, while the rest of the terms are due to the mean field pair-pair and pair-FS interactions.

The pump-induced indirect transition matrix element is given by

$$
\begin{aligned}
M_{\mathbf{p p}^{\prime} \mathbf{k}}(t) & =\left[\mathcal{P}_{e h}(\mathbf{k}, t)-\mathcal{P}_{e h}\left(\mathbf{p}+\mathbf{p}^{\prime}-\mathbf{k}, t\right)\right]^{*} \mathcal{P}_{e h}^{\mathrm{e}}\left(\mathbf{p} \mathbf{p}^{\prime} ; \mathbf{k} ; t\right) \\
& -\left[\mathcal{P}_{e h}(\mathbf{p}, t)+\mathcal{P}_{e h}\left(\mathbf{p}^{\prime}, t\right)\right]^{*}
\end{aligned}
$$




$$
\begin{aligned}
& \times\left[\mathcal{P}_{e h}^{\mathrm{e}}\left(\mathbf{p} \mathbf{p}^{\prime} ; \mathbf{p} ; t\right)+\mathcal{P}_{e h}^{\mathrm{h} *}\left(\mathbf{p} \mathbf{p}^{\prime} ; \mathbf{p}+\mathbf{p}^{\prime}-\mathbf{k} ; t\right)\right] \\
& +\mathcal{P}_{e h}\left(\mathbf{p}+\mathbf{p}^{\prime}-\mathbf{k}, t\right)\left[\mathcal{P}_{e h}^{\mathrm{e}}\left(\mathbf{k}, \mathbf{p}+\mathbf{p}^{\prime}-\mathbf{k} ; \mathbf{p}^{\prime} ; t\right)\right. \\
& \left.-\mathcal{P}_{e h}^{\mathrm{e}}\left(\mathbf{k}, \mathbf{p}+\mathbf{p}^{\prime}-\mathbf{k} ; \mathbf{p} ; t\right)\right]^{*} \\
& +\mathcal{P}_{e h}^{*}(\mathbf{k}, t)\left[\mathcal{P}_{e h}^{\mathrm{h}}\left(\mathbf{k}, \mathbf{p}+\mathbf{p}^{\prime}-\mathbf{k} ; \mathbf{p}^{\prime} ; t\right)+\mathcal{P}_{e h}^{\mathrm{e}}\left(\mathbf{p}, \mathbf{p}^{\prime} ; \mathbf{k} ; t\right)\right. \\
& \left.-\mathcal{P}_{e h}^{\mathrm{e}}\left(\mathbf{p}, \mathbf{p}^{\prime} ; \mathbf{p}+\mathbf{p}^{\prime}-\mathbf{k} ; t\right)-\mathcal{P}_{e h}^{\mathrm{h}}\left(\mathbf{k}, \mathbf{p}+\mathbf{p}^{\prime}-\mathbf{k} ; \mathbf{p} ; t\right)\right] \\
& +\mathcal{P}_{e h}(\mathbf{p}, t) \mathcal{P}_{e h}^{\mathrm{e}}\left(\mathbf{k}, \mathbf{p}+\mathbf{p}^{\prime}-\mathbf{k} ; \mathbf{p}^{\prime} ; t\right) \\
& -\mathcal{P}_{e h}\left(\mathbf{p}^{\prime}, t\right) \mathcal{P}_{e h}^{e *}\left(\mathbf{k}, \mathbf{p}+\mathbf{p}^{\prime}-\mathbf{k} ; \mathbf{p} ; t\right) .
\end{aligned}
$$

The effective $e-h$ potential is given by

$$
\begin{aligned}
v_{e h}\left(\mathbf{q} ; \mathbf{k k}^{\prime} ; t\right)=v(\mathbf{q})-\frac{\mu}{2} \mathcal{E}_{p}(t) & {\left[\mathcal{P}_{e h}^{e}\left(\mathbf{k}+\mathbf{q}, \mathbf{k}^{\prime} ; \mathbf{k}^{\prime}+\mathbf{q} ; t\right)\right.} \\
& +\mathcal{P}_{e h}^{h}\left(\mathbf{k}, \mathbf{k}^{\prime}+\mathbf{q} ; \mathbf{k}^{\prime} ; t\right) \\
& +\mathcal{P}_{e h}^{e *}\left(\mathbf{k}, \mathbf{k}^{\prime}+\mathbf{q} ; \mathbf{k}^{\prime} ; t\right) \\
& \left.+\mathcal{P}_{e h}^{h *}\left(\mathbf{k}+\mathbf{q}, \mathbf{k}^{\prime} ; \mathbf{k}^{\prime}+\mathbf{q} ; t\right)\right],
\end{aligned}
$$

and the effective $e-e$ potential is given by

$$
\begin{aligned}
v_{e e}\left(\mathbf{q} ; \mathbf{k k}^{\prime} ; t\right)=v(\mathbf{q})+\frac{\mu}{4} \mathcal{E}_{p}(t) & {\left[\mathcal{P}_{e h}^{e}\left(\mathbf{k}+\mathbf{q}, \mathbf{k}^{\prime}-\mathbf{q} ; \mathbf{k}^{\prime} ; t\right)\right.} \\
& -\mathcal{P}_{e h}^{e}\left(\mathbf{k}+\mathbf{q}, \mathbf{k}^{\prime}-\mathbf{q} ; \mathbf{k} ; t\right) \\
& +\mathcal{P}_{e h}^{e *}\left(\mathbf{k}, \mathbf{k}^{\prime} ; \mathbf{k}^{\prime}-\mathbf{q} ; t\right) \\
& \left.-\mathcal{P}_{e h}^{e *}\left(\mathbf{k}, \mathbf{k}^{\prime} ; \mathbf{k}+\mathbf{q} ; t\right)\right]
\end{aligned}
$$

\section{References}

[1] D. Pines and P. Nozieres, The theory of quantum liquids, (W. A. Benjamin, Inc., New York, 1966), Vol. I. 
[2] H. Haug and S. Schmitt-Rink, Prog. Quant. Electr. 9, 3 (1984).

[3] A. Stahl and I. Baslev, Electrodynamics of the Semiconductor band edge Springer Tracts in Modern Physics 110 (Springer-Verlag, Berlin, 1987).

[4] R. Zimmermann, Many-particle theory of highly excited semiconductors (Teubner, Leipzig, 1988).

[5] S. Schmitt-Rink, D. S. Chemla, and D. A. B. Miller, Adv. Phys. 38, 89 (1989).

[6] H. Haug and S. W. Koch, Quantum theory of the optical and electronic properties of semiconductors, 2nd edition (World Scientific, Singapore, 1993).

[7] S. Mukamel, Principles of Nonlinear Optical Spectroscopy (Oxford University Press, New York, 1995).

[8] R. Binder and S. W. Koch, Prog. Quant. Electron. 19, 307 (1995).

[9] J. Shah, Ultrafast Spectroscopy of Semiconductors and Semiconductor Nanostructures (Springer, New York, 1996).

[10] H. Haug and A.-P. Jauho, Quantum Kinetics in Transport and Optics of Semiconductors (Springer, 1996) and references therein.

[11] V. M. Axt and S. Mukamel, Rev. Mod. Phys. 70, 145 (1998).

[12] D. S. Chemla in Nonlinear Optics in Semiconductors, ed. by R. K. Willardson and A. C. Beers, 175 (Academic Press, 1999).

[13] Optical nonlinearities and instabilities in semiconductors, ed. by $\mathrm{H}$. Haug (Academic, Boston, 1988).

[14] Hot carriers in Semiconductor Nanostructures: Physics and Applications, ed. by J. Shah (Academic, Boston, 1992).

[15] Optics of Semiconductor Nanostructures, ed. by H. Henneberger, S. Schmitt-Rink, and E. O. Göbel (Akademie Verlag, Berlin, 1993).

[16] Coherent optical interactions in solids, ed. by R. T. Phillips (Plenum, New York, 1994). 
[17] H. Petek and S. Ogawa, Prog. Surf. Sci. 56, 239 (1997).

[18] T. Yajima and Y. Taira, J. Phys. Soc. Jpn. 47, 1620 (1979).

[19] L. Allen and J. H. Eberly, Optical Resonance and Two-Level Atoms (Dover, New York 1987).

[20] See e.g. R. Y. Shen, Principles of Nonlinear Optics (Wiley-Interscience, 1984).

[21] S. Schmitt-Rink and D. S. Chemla, Phys. Rev. Lett. 57, 2752 (1986).

[22] S. Schmitt-Rink, D.S. Chemla, and H. Haug, Phys. Rev. B 37, 941 (1988).

[23] M. Lindberg and S. W. Koch, Phys. Rev. B 38, 3342 (1988).

[24] I. Baslev, R. Zimmermann, and A. Stahl, Phys. Rev. B 40, 4095 (1989).

[25] R. Binder, S. W. Koch, M. Lindberg, W. Schäfer, and F. Jahnke, Phys. Rev. B 43, 6520 (1991).

[26] V. M. Axt and A. Stahl, Z. Phys. B 93, 195 (1994).

[27] Th. Östreich, K. Schönhammer, and L. J. Sham, Phys. Rev. Lett. 74, 4698 (1995).

[28] Th. Östreich, K. Schönhammer, and L. J. Sham, Phys. Rev. B 58, 12920 (1998).

[29] V. Chernyak, S. Yokojima, T. Meier, and S. Mukamel Phys. Rev. B 58, 4496 (1998); and references therein.

[30] I. V. Kukushkin et al., Phys. Rev. Lett. 72, 736 (1994).

[31] B. B. Goldberg et al., Phys. Rev. Lett. 65, 641 (1990).

[32] A. J. Turberfield et al., Phys. Rev. Lett. 65, 637 (1990).

[33] E. H. Aifer et al., Phys. Rev. Lett. 76, 680 (1996).

[34] P. W. Anderson, Phys. Rev. Lett. 18, 1049 (1967). 
[35] M. S. Skolnick, J. M. Rorison, K. J. Nash, D. J. Mowbray, P. R. Tapster, S. J. Bass, and A. D. Pitt, Phys. Rev. Lett 58, 2130 (1987).

[36] G. Livescu, D. A. B. Miller, D. S. Chemla, M. Ramaswamy, T. Y. Chang, N. Sauer, A. C. Gossard, and J. H. English, IEEE Journal of Quantum Electronics 24, 1677 (1988).

[37] W. Chen, M. Fritze, W. Walecki, A. Nurmikko, D. Ackley, M. Hong, L. L. Chang, Phys. Rev. B 45, 8464 (1992).

[38] J. M. Calleja, A. R. Goni, B. S. Dennis, J. S. Weiner, A. Pinczuk, S. Schmitt-Rink, L. N. Pfeiffer, K. West, J. F. Muller, and A. E. Ruckenstein, Solid State Comm. 79, 911 (1991).

[39] M. Fritze, A. Kastalsky, J. E. Cunningham, W. Knox, R. Partak, and I. E. Perakis, Solid State Com. 100, 497 (1996).

[40] e.g., G. D. Mahan in Fermi Surface Effects, ed. by J. Kondo and A. Yoshimori, 41 (Springer, Berlin, 1988).

[41] K. Ohtaka and Y. Tanabe, Rev. Mod. Phys. 62, 929 (1990).

[42] A. Ruckenstein and S. Schmitt-Rink, Phys. Rev. B 35, 7551 (1987).

[43] G. D. Mahan, Many-Particle Physics, Second Edition (Plenum, 1990).

[44] P. Nozieres and C. T. de Dominicis, Phys. Rev. 178, 1097 (1969).

[45] G. D. Mahan, Phys. Rev. 153, 882 (1967).

[46] G. D. Mahan, Phys. Rev. 163, 163 (1967).

[47] J. Gavoret, P. Nozieres, B. Roulet, and M. Combescot, J. Phys. (Paris) 30, 987 (1969).

[48] T. Uenoyama and L. J. Sham, Phys. Rev. Lett. 65, 1048 (1990).

[49] S. Schmitt-Rink, C. Ell, and H. Haug, Phys. Rev. B 33, 1183 (1983).

[50] K. Ohtaka and Y. Tanabe, Phys. Rev. B 39, 3054 (1989).

[51] P. Hawrylak, Phys. Rev. B 44, 3821 (1991). 
[52] H. Kummel, K. H. Luhrmann, and J. G. Zabolitzky, Phys. Rep. 36, 3 (1978)

[53] R. F. Bishop, Theor. Chim. Acta 80, 95 (1991).

[54] J. Arponen, Ann. Phys. (N. Y.) 151, 311 (1983).

[55] K. Schönhammer and O. Gunnarsson, Phys. Rev. B 18, 6606 (1978).

[56] I. E. Perakis and Yia-Chung Chang, Phys. Rev. B 44, 5877 (1991)

[57] I. E. Perakis and Yia-Chung Chang, Phys. Rev. B 43, 12556 (1991); Proc. 20th Int. Conf. Phys. Sem., ed. by E. M. Anastassakis and J. D. Joannopoulos, 1109 (World Scientific, Singapore, 1990).

[58] I. E. Perakis and Yia-Chung Chang, Phys. Rev. B 47, 6573 (1993).

[59] K. Emrich and J. G. Zabolitzky, Phys. Rev. B 30, 2049 (1984).

[60] R. F. Bishop and K. H. Luhrmann, Phys. Rev. B 17, 3757 (1978); ibid. 26, 5523 (1982).

[61] J. W. Negele, Rev. of Mod. Phys. 54, 913 (1982).

[62] W, H. Knox, D. S. Chemla, G. Livescu, J. E. Cunningham, and J. E. Henry, Phys. Rev. Lett. 61, 1290 (1988).

[63] H. Wang, J. Shah, T. C. Damen, S. W. Pierson, T. L. Reinecke, L. N. Pfeiffer, and K. West, Phys. Rev. B 52, R17013 (1995).

[64] A. Tomita, J. Shah, J. E. Cunningham, S. M. Goodnick, P. Lugli, and S. L. Chuang, Phys. Rev. B 48, 5708 (1993).

[65] A. Chebira, J. Chesnoy, and G. M. Gale, Phys. Rev. B 46, 4559 (1992).

[66] X. Q. Zhou, K. Leo, and H. Kurz, Phys. Rev. B 45, 3886 (1992).

[67] M. Woerner, W. Frey, M. T. Portella, C. Ludwig, T. Elsaesser, and W. Kaiser, Phys. Rev. B 49, 17007 (1994)

[68] M. Woerner and T Elsaesser, Phys. Rev. B 51, 17490 (1995).

[69] See e.g. J. Faist, F. Capasso, D. L. Sivco, C. Sintori, A. L. Hutchinson, and A. Y. Cho, Science 264, 553 (1994). 
[70] S. Lutgen, R. A. Kaindl, M. Woerner, T. Elsaesser, A. Hase, H. Künzel, M. Gulia, D. Meglio, and P. Lugli, Phys. Rev. Lett. 77, 3657 (1996).

[71] S. Lutgen, R. A. Kaindl, M. Woerner, T. Elsaesser, A. Hase, and H. Künzel, Phys Rev B 54, R17343 (1996).

[72] M. C. Tatham, J. F. Ryan, and C. T. Foxon, Phys. Rev. Lett. 63, 1637 (1989).

[73] A. Seilmeier, H.-J. Hübner, G. Abstreiter, G. Weimann, and W. Schlapp, Phys. Rev. Lett. 59, 1345 (1987).

[74] P. Hawrylak, J. F. Young, and P. Brockmann, Phys. Rev. B 49, 13624 (1994).

[75] See e.g. A. F. J. Levi, Electron. Lett. 124, 1273 (1988).

[76] D-S Kim, J. Shah, J. E. Cunningham, T. C. Damen, S. Schmitt-Rink, and W. Schäfer, Phys. Rev. Lett. 68, 2838 (1992).

[77] S. Bar-Ad, I. Bar-Joseph, Y. Levinson, and H. Shtrikman, Phys. Rev. Lett. 72, 776 (1994).

[78] R. A. Kaindl, S. Lutgen, M. Woerner, T. Elsaesser, B. Nottelmann, V. A. Axt, T. Kuhn, A. Hase, and H. Künzel, Phys. Rev. Lett. 80, 3575 (1998).

[79] A. Bonvalet, J. Nagle, V. Berger, A. Migus, J.-L. Martin, and M. Joffre, Phys. Rev. Lett. 76, 4392 (1996).

[80] See e.g. J. E. Bjorkholm and P. F. Liao, Phys. Rev. Lett. 33, 128 (1974); P. F. Liao and J. E. Bjorkholm, ibid 34, 1 (1975).

[81] M. Combescot, Phys. Rep. 221, 167 (1992).

[82] I. Brener, W. H. Knox, and W. Schaefer, Phys. Rev. B 51, 2005 (1995).

[83] I. E. Perakis, I. Brener, W. H. Knox, and D. S. Chemla, J. Opt. Soc. Am. B 13, 1313 (1996); and in Ultrafast Phenomena IX, ed. by P. F. Barbara, W. H. Knox, G. A. Mourou, and A, H. Zewail, 407 (SpringerVerlag, Berlin, 1994). 
[84] M.-A. Mycek, J.-Y. Bigot, I. E. Perakis, and D. S. Chemla, J. Opt. Soc. Am. B 13, 1284 (1996); M.-A. Mycek, J.-Y. Bigot, I. E. Perakis, and D. S. Chemla, J. Nonlin. Opt. Phys. Mater. 4, 497 (1995).

[85] N. A. Fromer, C. Schüller, D. S. Chemla, T. V. Shahbazyan, I. E. Perakis, K. Maranowski, and A. C. Gossard, Phys. Rev. Lett. 1999.

[86] S. Girvin et al., Phys. Rev. B 33, 2481 (1986).

[87] R. Haussmann, Phys. Rev. B 53, 7357 (1996).

[88] I. V. Lerner and Yu. E. Lozovik, Zh. Exp. Teor. Fiz. 80, 1488 (1981) [Sov. Phys.-JETP 53, 763 (1981)].

[89] C. Jung, S. Yee, and K. Kuhn, Applied Optics 34, 946 (1995).

[90] J. W. Haus and N. Kalyaniwalla, J. Appl. Phys. 65, 1420 (1989).

[91] C. P. Collier, R. J. Saykally, J. J. Shiang, S. E. Henrichs, and J. R. Heath, Science 277, 1978 (1997).

[92] L. Landin, M. S. Miller, M. E. Pistol, C. E. Pryor, and L. Samuelson, Science 280, 262 (1998).

[93] D. C. Ralph, C. T. Black, and M. Tinkham, Phys. Rev. Lett. 78, 4087 (1997).

[94] B. Liedberg, I. Lundstrom, and E. Stenberg, Sensors and Actuators B-Chemical, 11, 63 (1993).

[95] I. Lundstrom, Biosensors \& Bioelectronics, 9, 725 (1994).

[96] E. M. Phizicky and S. Fields, Microbiological Reviews 59, 94 (1995).

[97] P. M. Richaletsecordel, F. Poisson, and MH. V. Vanregenmortel, Clinical and Diagnostic Virology 5, 111 (1996).

[98] C. E. H. Berger, R. P. H. Kooyman, and J. Greve, Review Of Scientific Instruments 65, 2829 (1994).

[99] W. A. De Heer, Rev. Mod. Phys. 65, 611 (1993).

[100] M. Brack, Rev. Mod. Phys. 65, 677 (1993). 
[101] J. A. Perenboom, P. Wyder, and M. Meier, Phys. Rep. 78, 173 (1981).

[102] W. P. Halperin, Rev. Mod. Phys. 58, 533 (1986).

[103] U. Kreibig and M. Vollmer, Optical Properties of Metal Clusters (Springer, Berlin, 1995), and references therein.

[104] G. Mie, Ann. Phys. 25, 377 (1908).

[105] J. C. Maxwell Garnett, Phil. Trans. Royal Soc. A 203, 385 (1906).

[106] C. F. Bohren and D. R. Huffman, Absorption and Scattering of light by small particles (Wiley, New York, 1983).

[107] A. Kawabata and R. Kubo, J. Phys. Soc. Japan 211765 (1966).

[108] F. Hache, D. Ricard, and C. Flytzanis, J. Opt. Soc. Am. B 31647 (1986).

[109] See e.g. C. Flytzanis, F. Hache, M. C. Klein, D. Ricard, and Ph. Roussignol, in Progress in Optics XXIX, ed. by E. Wolf, 321 (Elsevier, Amsterdam, 1991).

[110] L. Yang, K. Becker, F. M. Smith, R. H. Magruder, R. F. Haglund, L. Yang, R. Dorsinville, R. R. Alfano, and R. A. Zuhr, J. Opt. Soc. Am. B 11457 (1994).

[111] G. S. Agarwal and S. D. Gupta, Phys. Rev. A 385678 (1988).

[112] The effects of the spill out of the electron wave-functions beyond the nanoparticle classical boundary were discussed, e.g., in V. V. Krezin, Phys. Rep. 220, 1 (1992).

[113] See, e.g., Physics and Chemistry of Finite Systems: From clusters to Crystals, edited by P. Jena et al. (NATO Advanced Study Institute Series C, Kluwer Academic, Dordrecht/Boston, 1992).

[114] U. Sivan, Y. Imry, and A. G. Aronov, Europhys. Lett. 28, 115 (1994).

[115] In semiconductor quantum dots, where the discrete energy levels are well resolved, the quasiparticle scattering rate is similar to that in the bulk only for energies larger than some critical energy; see, e.g., B. I. 
Altshuler, Y. Gefen, A. Kamenev, and L. S. Levitov, Phys. Rev. Lett. 78, 2803 (1997).

[116] T. Klar, M. Perner, S. Grosse, G. von Plessen, W. Spirkl, and J. Feldmann, Phys. Rev. Lett. 80, 4249 (1998).

[117] B. Lamprecht, J. R. Krenn, A. Leitner, and F. R. Aussenegg, Phys. Rev. Lett. 83, 4421 (1999).

[118] J. H. Klein-Wiele, P. Simon, and H. G. Rubahn, Phys. Rev. Lett. 80, 45 (1998).

[119] W. S. Fann, R. Storz, W. H. K. Tom, and J. Bokor, Phys. Rev. Lett. 68, 2834 (1992).

[120] W. S. Fann, R. Storz, W. H. K. Tom, and J. Bokor, Phys. Rev. B 46, 13592 (1992).

[121] R. H. M. Groeneveld, R. Sprik, A. Lagendijk, Phys. Rev. B 51, 11433 (1995).

[122] R. H. M. Groeneveld, R. Sprik, A. Lagendijk, Phys. Rev. B 45, 5079 (1992).

[123] C. K. Sun, F. Vallee, L. H. Acioli, E. P. Ippen, and F. G. Fujimoto, Phys. Rev. B 48, 12365 (1993).

[124] C. K. Sun, F. Vallee, L. H. Acioli, E. P. Ippen, and F. G. Fujimoto, Phys. Rev. B 50, 15337 (1994).

[125] N. Del Fatti, R. Bouffanais, F. Vallée, and C. Flytzanis, Phys. Rev. Lett. 81, 922 (1998).

[126] J.-Y. Bigot, J.-C. Merle, O. Cregut, and A. Daunois, Phys. Rev. Lett. 75, 4702 (1995).

[127] M. Perner, P. Bost, G. von Plessen, J. Feldmann, U. Becker, M. Mennig, M. Schmitt, and H. Schmidt Phys. Rev. Lett. 78, 2192 (1997).

[128] V. Halte, J. Guille, J.-C. Merle, I. Perakis, and J.-Y. Bigot, Phys. Rev. B 60, 11738 (1999). 
[129] R. D. Averitt, S. L. Westcott, and N. J. Halas, Phys. Rev. B 58, R10203 (1998).

[130] R. D. Averitt, S. L. Westcott, and N. J. Halas, J. Opt. Soc. Am. B 16, 1814 (1999).

[131] E. D. Belotskii, S. N. Luk'yanets, and P. M. Tomchuk, Zh. Eksp. Teor. Fiz. 101, 163 (1992) [Sov. Phys. JETP 74, 88 (1992)].

[132] T. Tokizaki, A. Nakamura, S. Kaneko, K. Uchida, S. Omi, H. Tanji, and Y. Asahara, Appl. Phys. Lett. 65, 941 (1994).

[133] T. S. Ahmadi, S. L. Logunov, and M. A. Elsayed, J. Phys. Chem. 100, 8053 (1996).

[134] S. Link and M. A. El-Sayed, J. Phys. Chem B 103, 8410 (1999).

[135] B. A. Smith, J. Z. Zhang, U. Giebel, and G. Schmid, Chem Phys. Lett. 270, 139 (1997).

[136] T. W. Roberti, B. A. Smith, and J. Z. Zhang, J. Chem. Phys. 102, 3860 (1995).

[137] H. Inouye, K. Tanaka, I. Tanahashi, and K. Hirao, Phys. Rev. B 57, 11334 (1998).

[138] J. Hodak, I. Martini, and G. V. Hartland, Chem. Phys. Lett. 284, 135 (1998).

[139] R. N. Gurzhi, Sov. Phys. JETP 35, 673 (1959).

[140] M. Kaveh and N. Wiser, Adv. Phys. 33, 257 (1984).

[141] M. I. Kaganov, I. M. Lifshitz, and L. V. Tanatarov, Sov. Phys. JETP 4, 173 (1957).

[142] S. I. Anisimov, B. L. Kapeliovich, and T. L. Prel'man, Sov. Phys. JETP 39, 375 (1975).

[143] P. B. Allen, Phys. Rev. Lett. 59, 1460 (1987).

[144] G. L. Eesley, Phys. Rev. Lett. 51, 2140 (1983). 
[145] R. W. Schoenlein, W. Z. Lin, J. G. Fujimoto, and G. L. Eesley, Phys. Rev. Lett. 58, 1680 (1987).

[146] C. Suarez, W. E. Bron, and T. Juhasz, Phys. Rev. Lett. 75, 4536 (1995).

[147] H. E. Elsayed-Ali, T. B. Norris, M. A. Pessot, and G. A. Mourou, Phys. Rev. Lett. 58, 1212 (1987).

[148] A. Stella, M. Nisoli, S. De Silvestri, O. Svelto, G. Lanzani, P. Cheyssac, and R. Kofman, Phys. Rev. B 53, 15497 (1996).

[149] M. Nisoli, S. Stagira, S. De Silvestri, A. Stella, P. Tognini, P. Cheyssac, and R. Kofman, Phys. Rev. Lett. 78, 3575 (1997).

[150] E. D. Belotskii and P. M. Tomchuk, Int. J. Electron. 73, 955 (1992).

[151] V. Halte, B. Palpant, B. Prevel, J. C. Merle, M. Broyer, A. Perez, and J.-Y. Bigot, in Ultrafast Phenomena XI, ed. by T. Elsaesser, J. G. Fujimoto, D. A. Wiersma, and W. Zinth (Springer, Berlin, 1998).

[152] T. V. Shahbazyan, I. E. Perakis, and J.-Y. Bigot, Phys. Rev. Lett. 81, 3120 (1998); and in Trends in Optics and Photonics: Radiative Processes and Dephasing in Semiconductors, TOPS Vol. XVIII, Opt. Soc. of Am. 17 (Washington DC, 1998).

[153] T. V. Shahbazyan and I. E Perakis, Phys. Rev. B 60, 9090 (1999); and in Chem. Phys. special issue on "Electron Dynamics in Metals", ed. by H. Petek and T. Heinz (in press).

[154] I. E. Perakis and T. V. Shahbazyan, Int. J. Mod. Phys. B 13, 869 (1999).

[155] I. E. Perakis and D. S. Chemla, Phys. Rev. Lett. 72, 3202 (1994).

[156] I. E. Perakis, Chem. Phys. 210, 259 (1996).

[157] N. Primozich, T. V. Shahbazyan, I. E. Perakis, and D. S. Chemla, Phys. Rev. B (in press).

[158] J. R. Schrieffer and P. A. Wolff, Phys. Rev. 149, 491 (1966); J. R. Schrieffer, J. Appl. Phys. 38, 1143 (1967). 
[159] I. Shavitt and L. Redmon, J. Chem Phys. 73, 5711 (1980); and references therein.

[160] C. Cohen-Tannoudji, J. Dupont-Roc, and G. Grynberg, Atom-Photon Interactions: Basic Processes and Applications (John Wiley \& Sons, Inc., 1992).

[161] An analogous treatment of the interplay between the interband transitions and the $e-h$ interactions was considered in a different context in I. E. Perakis and C. M. Varma, Phys. Rev. B 49, 9041 (1994); I. E. Perakis, C. M. Varma, and A. E. Ruckenstein, Phys. Rev. Lett. 70, 3467 (1993).

[162] E. Müller-Hartmann, T. V. Ramakrishnan, and G. Toulouse, Phys. Rev. B 3, 1102 (1971).

[163] A good discussion of the physics may be found in G. D. Mahan, Solid State Phys. 29, ed. H. Ehrenreich, F. Seitz, and D. Turnbull (Academic, New York) 75 (1974).

[164] P. W. Anderson, Phys. Rev. 112, 1900 (1958); D. Pines, Elementary Excitations in Solids (Addison-Wesley, 1963).

[165] P. Hawrylak, Phys. Rev. B 42, 8986 (1990).

[166] K. El Sayed, S. Schuster, H. Haug, F. Herzel, and K. Hennerberger, Phys. Rev. B 49, 7337 (1994).

[167] L. Bányai, Q. T. Vu, B. Mieck, and H. Haug, Phys. Rev. Lett. 81, 882 (1998).

[168] See, e.g., J. Igarashi, J. Phys. Soc. Jpn 54, 260 (1985).

[169] W. Chen, M. Fritze, A. V. Nurmikko, D. Ackley, C. Colvard, and H. Lee, Phys. Rev. Lett. 64, 2434 (1990).

[170] W. Chen, M. Fritze, A. V. Nurmikko, J. M. Hong, and L. Chang, Phys. Rev. B 43, 14738 (1991).

[171] J. F. Mueller, A. E. Ruckenstein, and S. Schmitt-Rink, Mod. Phys. Lett. B 5, 135 (1990). 
[172] J. F. Mueller, Phys. Rev. B 42, 11189 (1991).

[173] P. Hawrylak, Phys. Rev. B 44, 6262 (1991).

[174] M. S. Skolnick, et al., Phys. Rev. B 43, 7354 (1991).

[175] W. Knox, D. S. Chemla, D. A. B. Miller, J. Stark, and S. Schmitt-Rink, Phys. Rev. Lett. 62, 1189 (1989).

[176] D.S. Chemla, W.H. Knox, D.A.B. Miller, S. Schmitt-Rink, J.B. Stark, and R. Zimmermann, J. Lumin. 44, 233 (1989).

[177] C. Ell, J. F. Muller, K. El Sayed, and H. Haug, Phys. Rev. Lett. 62, 304 (1989).

[178] M. Combescot, and C. Tanguy, Phys. Rev. B 44, 3762 (1991).

[179] B. Fluegel, N. Peyghambarian, G. Olbright, M. Lindberg, S. W. Koch, M. Joffre, D. Hulin, A. Migus, A. Antonetti Phys. Rev. Lett. 59, 2588 (1987).

[180] T. V. Shahbazyan, I. E. Perakis, and D. S. Chemla, Phys. Rev. Lett. (in press).

[181] A. A. Lushnikov and A. J. Simonov, Z. Phys. 270, 17 (1974).

[182] In very small particles, the difference in the positions of the effective boundaries for the conduction and $d$-band densities leads to a shift in the linear absorption peak; see A. Liebsch, Phys. Rev. B 48, 11317 (1993); V. V. Krezin, Phys. Rev. B 51, 1844 (1995).

[183] S. Ogawa, H. Nagano, and H. Petek, Phys. Rev. B 55, 10869 (1997).

[184] H. Ehrenreich and H. R. Philipp, Phys. Rev. 128, 1622 (1962).

[185] S. Dodge, et al., Phys. Rev. Lett. 83, 4650 (1999).

[186] T. Portengen, Th. Ostreich, and L. J. Sham, Phys. Rev. Lett. 76, 3384 (1996); Phys. Rev. B 54, 17452 (1996).

[187] T. V. Shahbazyan, I. E. Perakis, and M. E. Raikh, Phys. Rev. Lett. (submitted). 
[188] G. Ju, A. V. Nurmikko, R. F. C. Farrow, R. F. Marks, M. J. Carey, and B. A. Gurney, Phys. Rev. Lett. 82, 3705 (1999).

[189] E. Beaurepaire, J.-C. Merle, A. Daunois, and J.-Y. Bigot, Phys. Rev. Lett. 76, 4250 (1996).

[190] W. Nessler, S. Ogawa, H. Nagano, H. Petek, J. Shimoyama, Y. Nakayama, and K. Kishio, Phys. Rev. Lett. 81, 4480 (1998). 


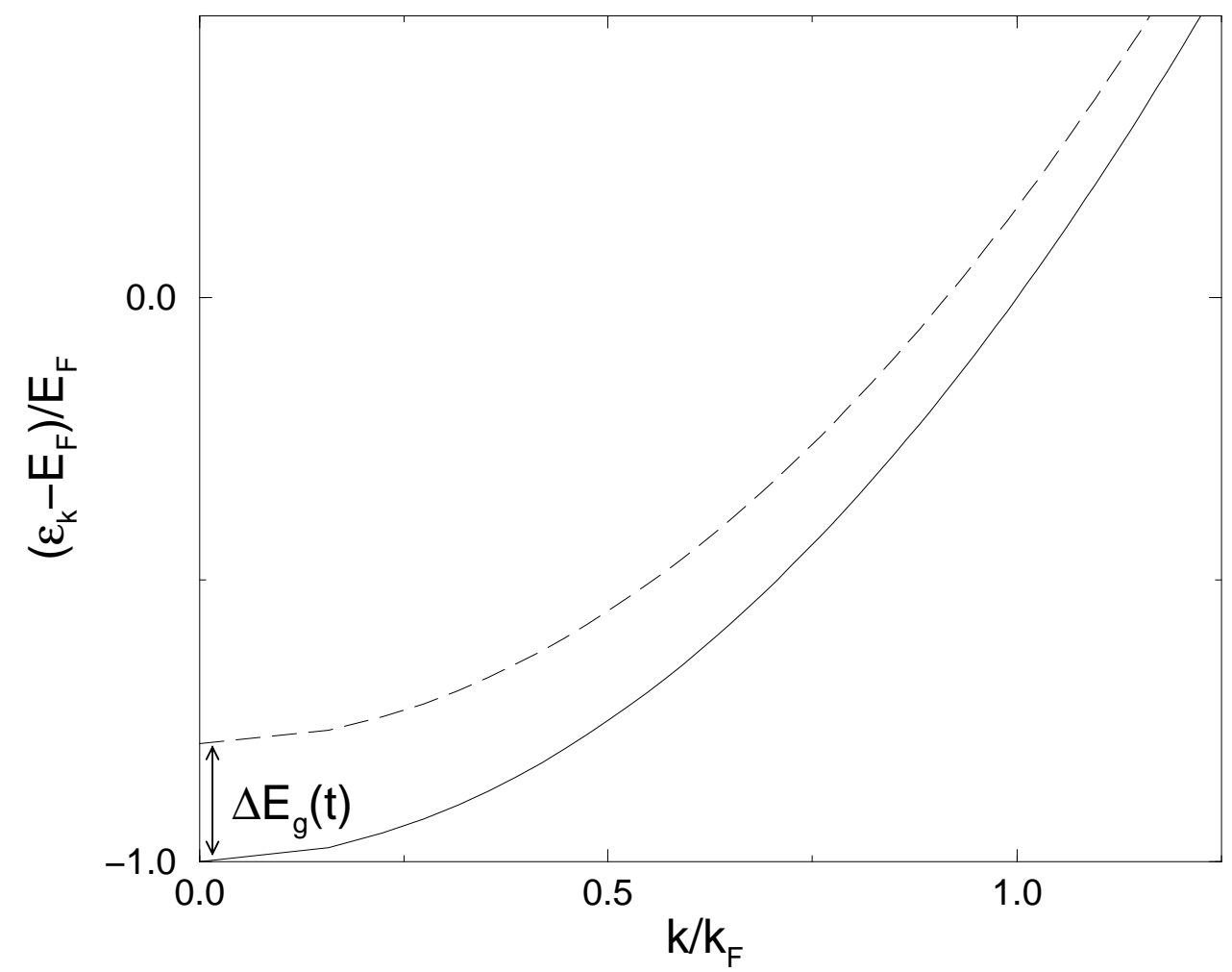

Figure 1: The effect of the pump pulse on the band dispersion, $\varepsilon_{\mathbf{k}}(t)=$ $\varepsilon_{\mathbf{k}}^{c}(t)+\varepsilon_{-\mathbf{k}}^{v}(t)$, of the "pump-dressed" system. Solid line: "bare" dispersion $(t=-\infty)$. Dashed line: pump-renormalized dispersion $(t=0)$. The bands become "heavier" for the duration of the pump. 
(a)

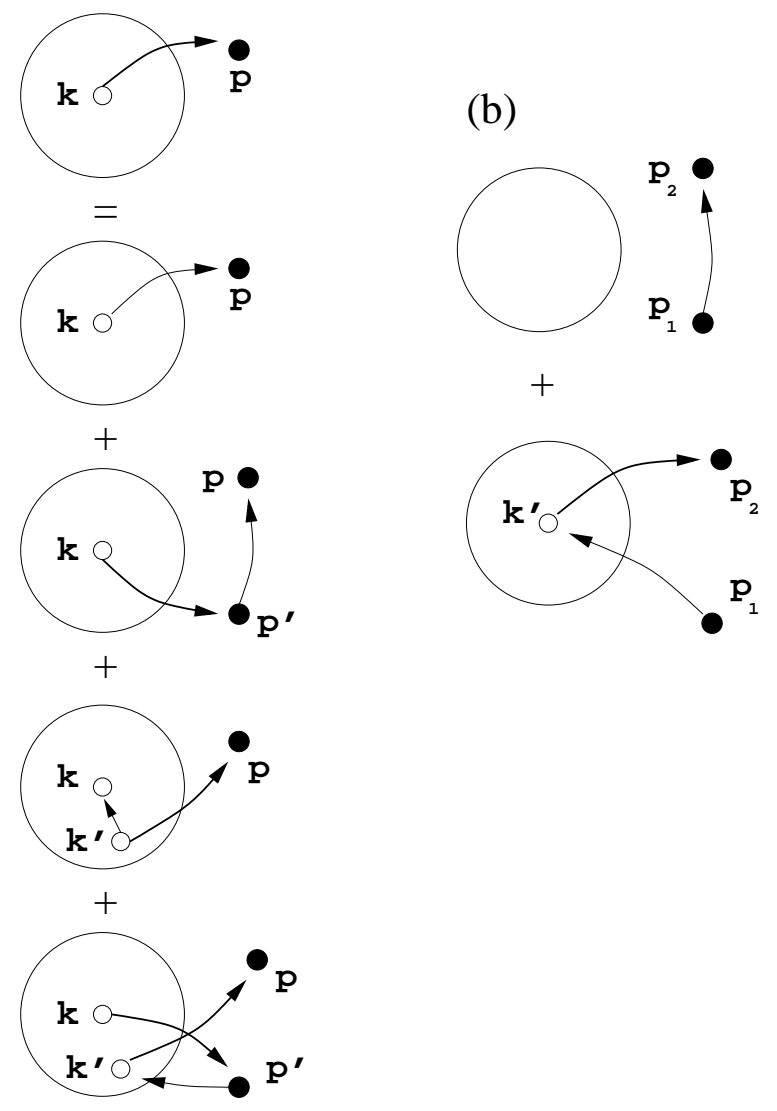

Figure 2: (a) The $e-h$ scattering processes that contribute to the rhs of Eq. (39). Full lines correspond to $s(\mathbf{p}, \mathbf{k}, t)$ and thin lines to $V$. The diagrams describe (from top to bottom) (i) Born scattering of a FS electron, (ii) FS electron ladder diagrams, (iii) FS hole ladder diagrams, and (iv) Nonlinear vertex corrections due to the dynamical FS response. (b) Scattering processes state that determine the time- and momentum-dependence of the effective $e-h$ potential $\tilde{V}(\mathbf{p}, t)$ and lead to the unbinding of the HFA bound state. 


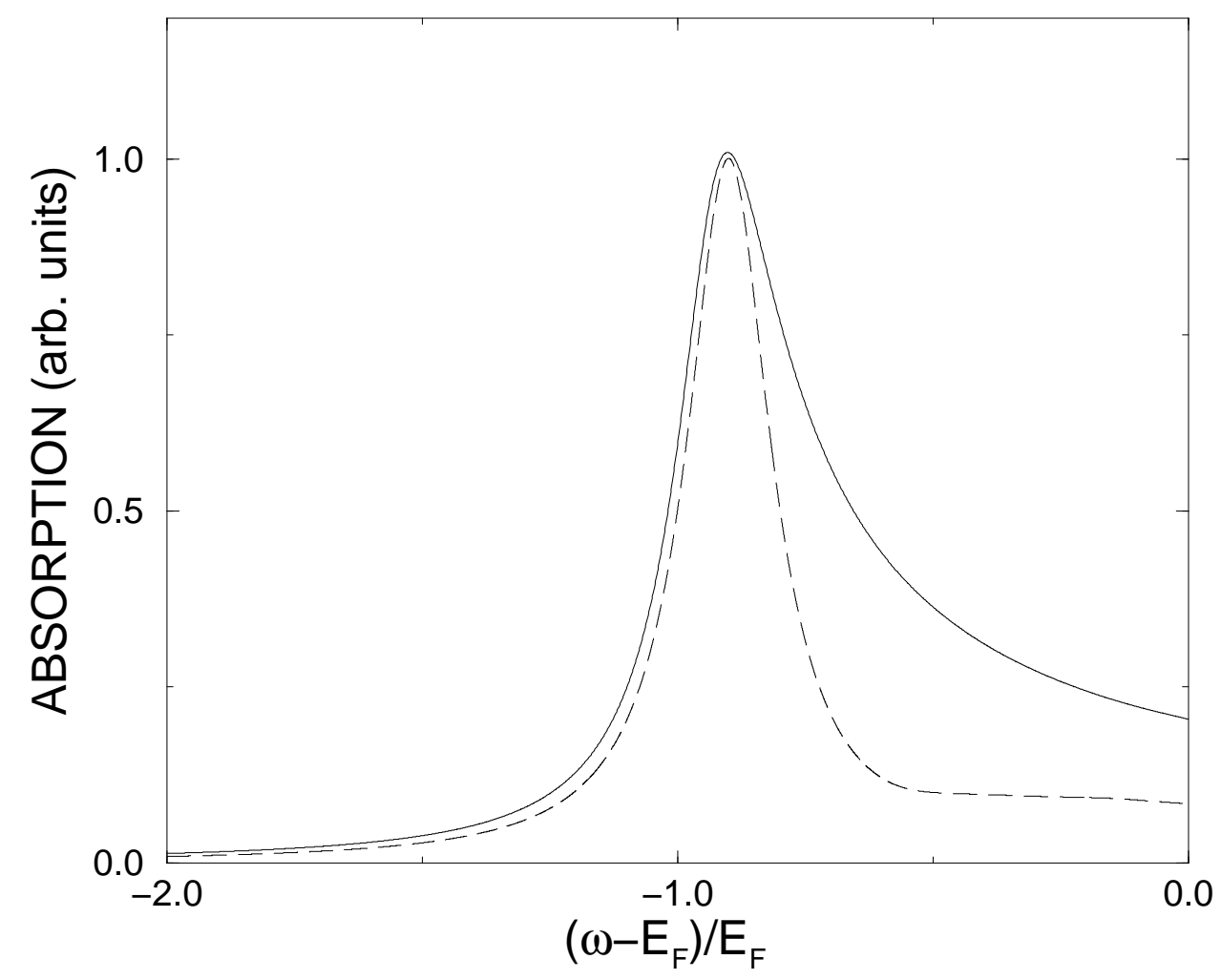

Figure 3: Linear absorption resonance lineshape for FES (solid curve) compared to the HFA (dashed curve), calculated with $g=0.4$ and $\Gamma=0.1 E_{F}$. The HFA resonance position was shifted for better visibility. 


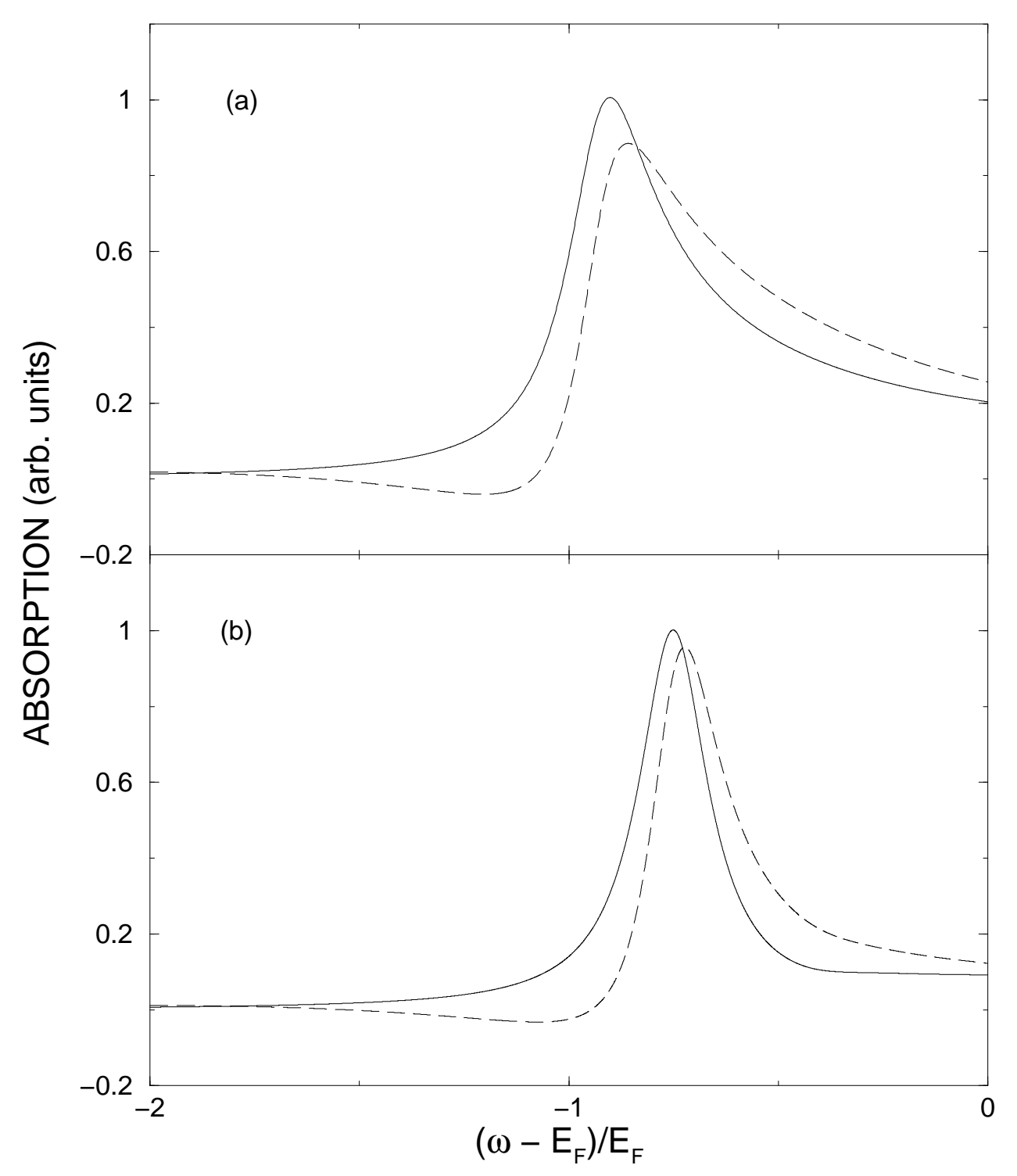

Figure 4: The absorption spectra for the FES (a) compared to the HFA (b). Solid curves: linear absorption. Dashed curves: nonlinear absorption. The curves were calculated with $g=0.4$ and $\Gamma=0.1 E_{F}$ at time delay $\tau=-0.1 \Gamma^{-1}=-t_{p} / 2$ and pulse duration $t_{p}=2.0 E_{F}^{-1}$. The nonlinear absorption lineshapes exhibit bleaching, resonance blueshift, and gain below the absorption onset that differ in the two cases. 


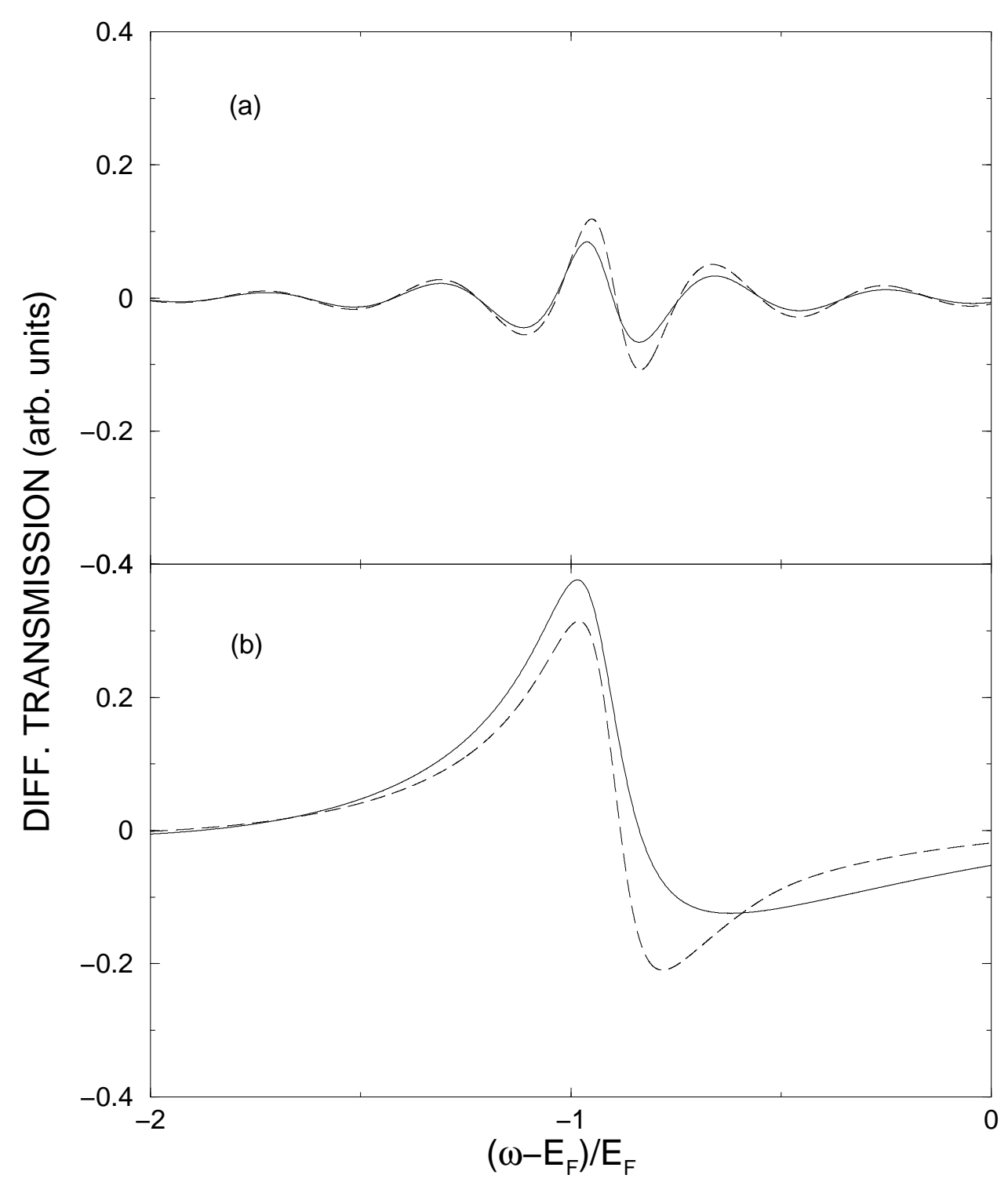

Figure 5: Differential transmission lineshape for various time delays calculated with $g=0.4, \Gamma=0.1 E_{F}$, and $t_{p}=2.0 E_{F}^{-1}$. (a) Long time delay, $\tau=-1.5 \Gamma^{-1}=-15.0 E_{F}^{-1}$. For the FES, the oscillations in the differential transmission spectra are reduced (solid curve) as compared to the HFA (dashed curve). (b) Short time delay, $\tau=-0.1 T_{2}=-t_{p} / 2=-1.0 E_{F}^{-1}$. For the FES, the differential transmission spectrum is asymmetric (solid curve) as compared to the symmetric lineshape for the HFA (dashed curve). The above curves were shifted for better visibility. 


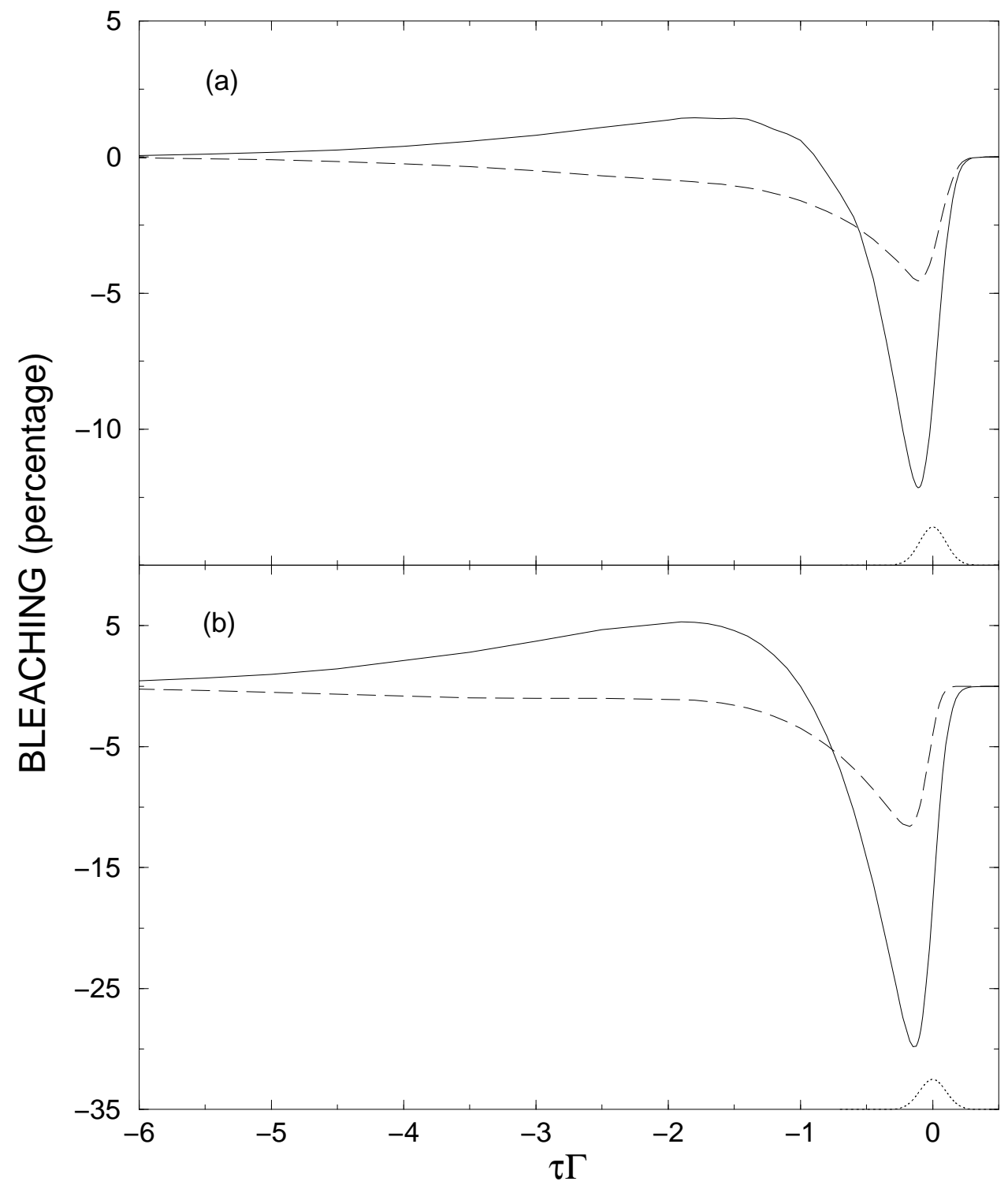

Figure 6: (a) Nonlinear absorption resonance bleaching evaluated at the instantaneous peak frequency as function of time delay for the FES (solid curve) compared to the HFA (dashed curve). The curves were calculated with $g=0.4, \Gamma=0.1 E_{F}$, and $t_{p}=2.0 E_{F}^{-1}$. The time-dependence of the pump pulse is also presented for comparison (dotted curve). (b) Same calculated using the rigid band shift model, Eq. (59). 


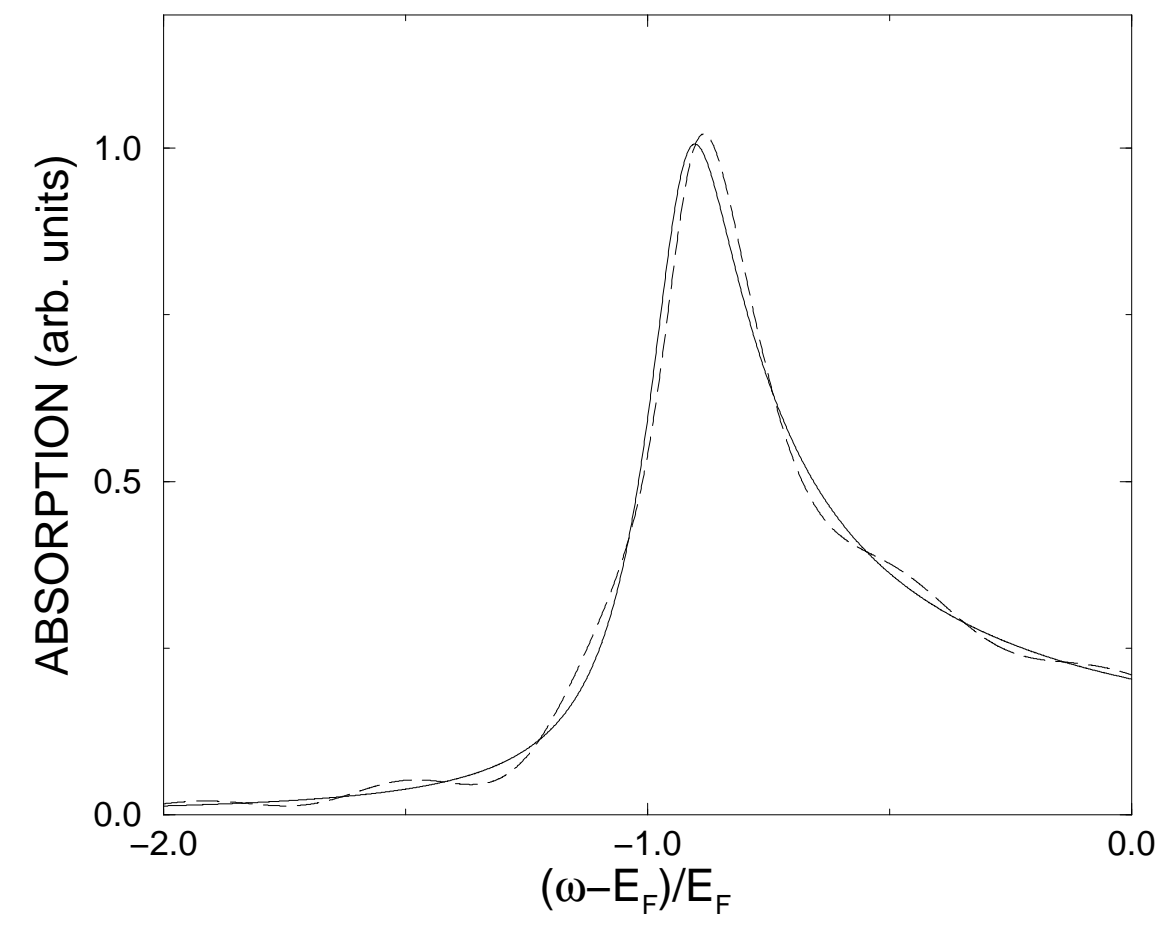

Figure 7: The enhancement of the nonlinear absorption resonance of the FES (dashed curve) vs. linear absorption (solid curve) at long time delay $\tau=-1.5 \Gamma^{-1}$. The curves were calculated with $g=0.4, \Gamma=0.1 E_{F}$, and $t_{p}=2.0 E_{F}^{-1}$. 


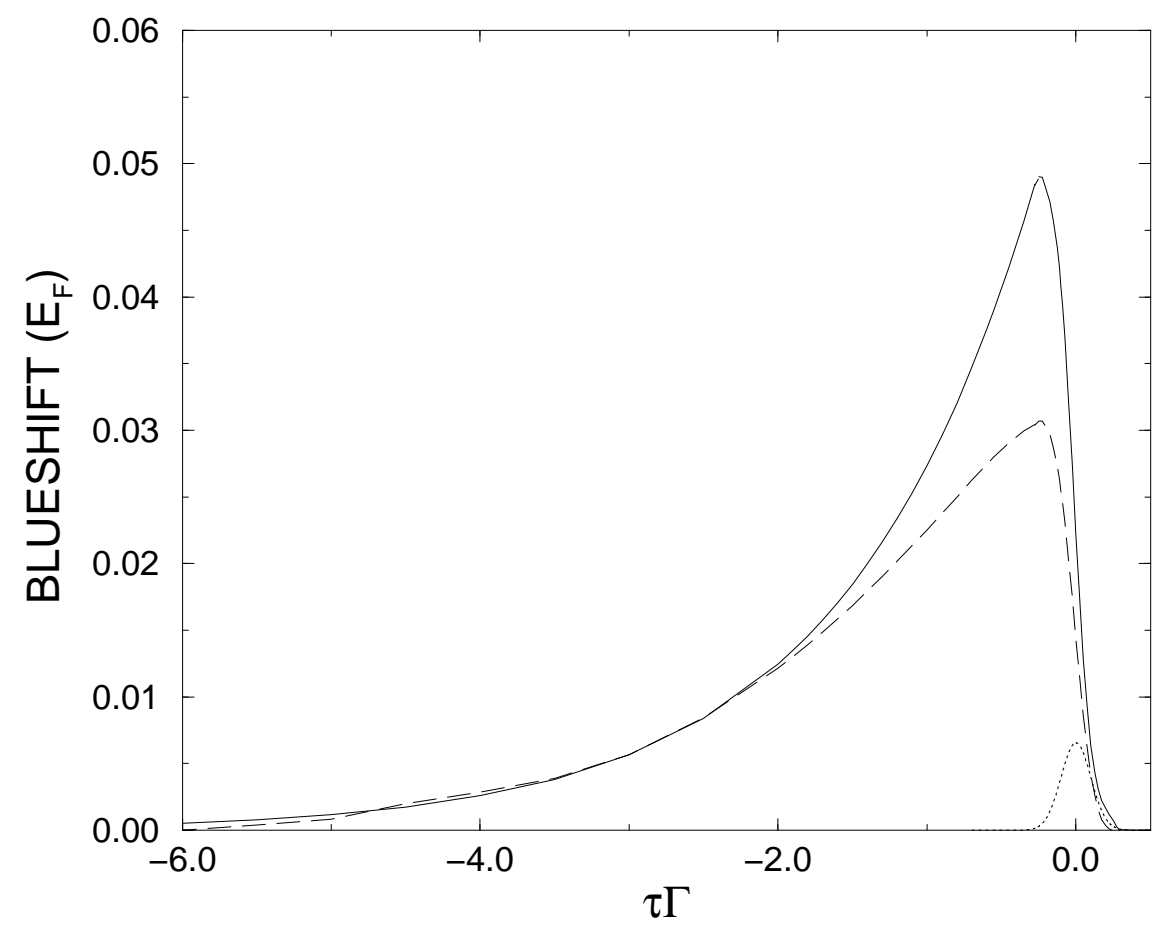

Figure 8: Nonlinear absorption resonance blueshift as function of time delay for the FES (solid curve) compared to the HFA (dashed curve). The blueshift is significantly weaker for the HFA. The curves were calculated with $g=0.4$, $\Gamma=0.1 E_{F}$, and $t_{p}=2.0 E_{F}^{-1}$. The time-dependence of the pump pulse is also presented for comparison (dotted curve). 


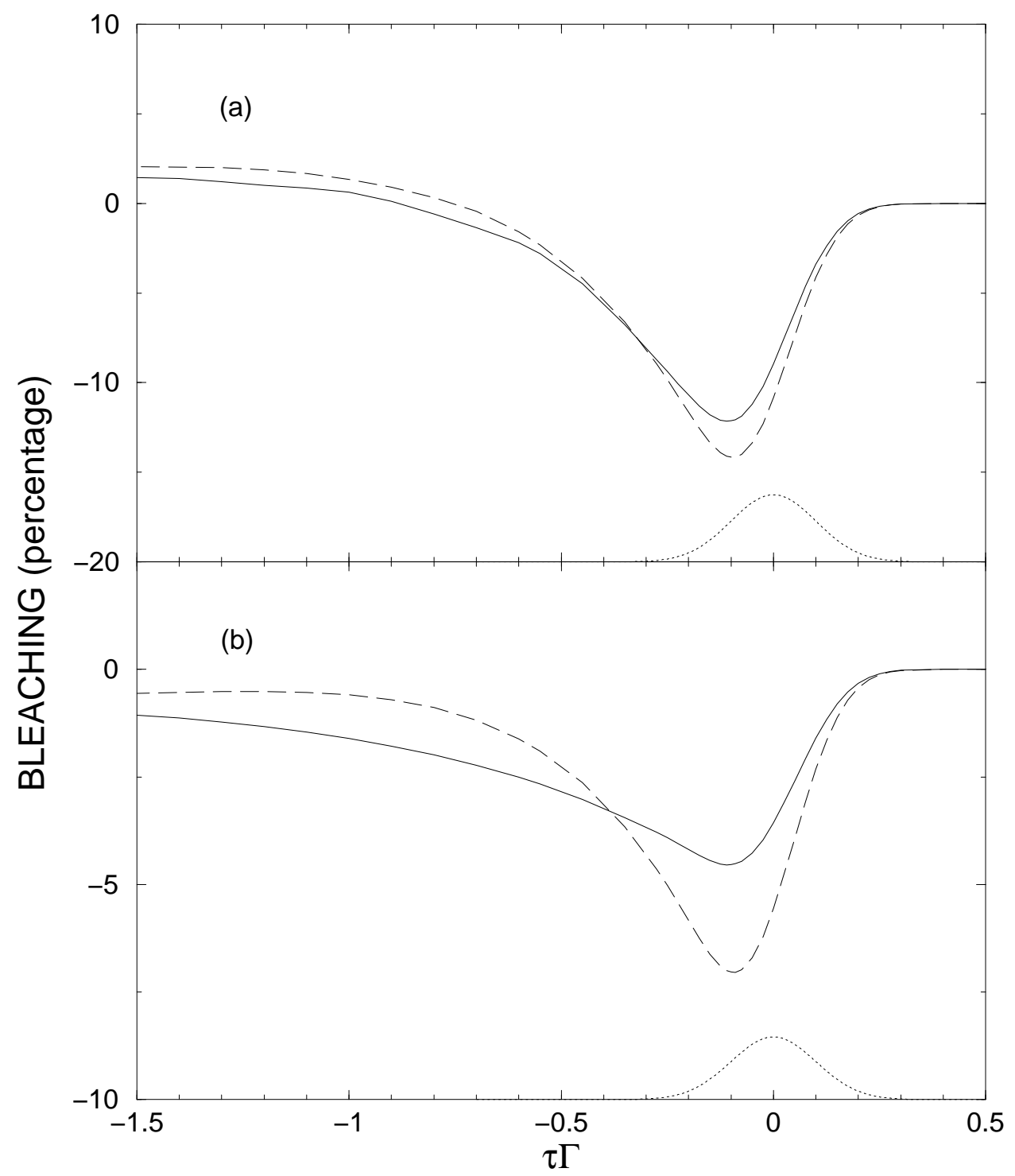

Figure 9: Resonance bleaching as function of time delay for the FES (a) compared to the HFA (b) for different strengths of the $e-h$ interaction: $g=0.4$ (solid curve) and $\mathrm{g}=0.3$ (dashed curve). The curves were calculated with $\Gamma=0.1 E_{F}$, and $t_{p}=2.0 E_{F}^{-1}$. The time-dependence of the pump pulse is also presented for comparison (dotted curve). 


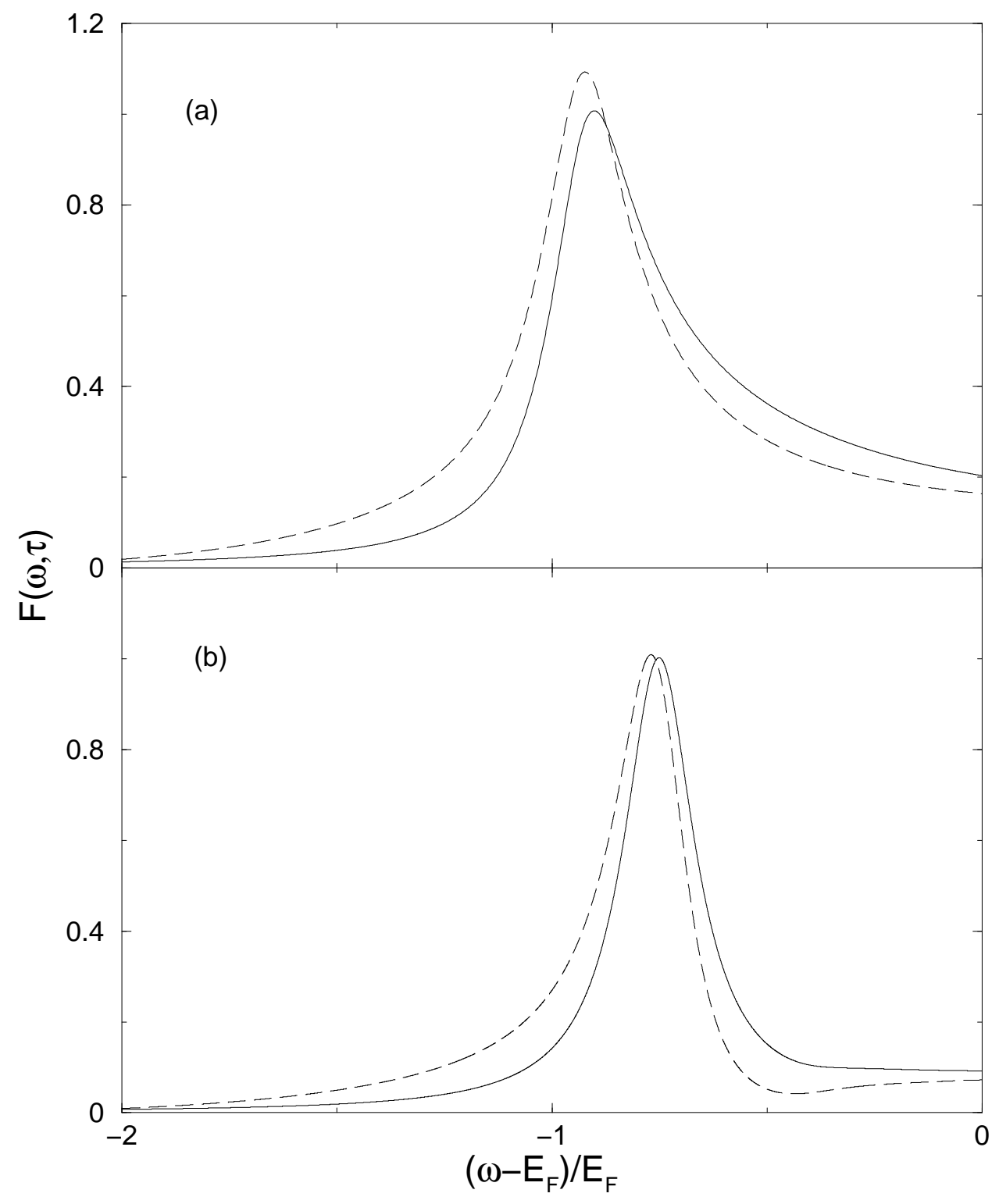

Figure 10: The effect of the pump-induced renormalization of the band dispersions on the $e-h$ interactions. The function $F(\omega, \tau)$, given by Eq. (60), for the FES (a) compared to the HFA (b) in the presence (dashed curve) and absence (solid curve) of the pump pulse. The curves were calculated with $g=0.4, \Gamma=0.1 E_{F}$, and $t_{p}=2.0 E_{F}^{-1}$. 


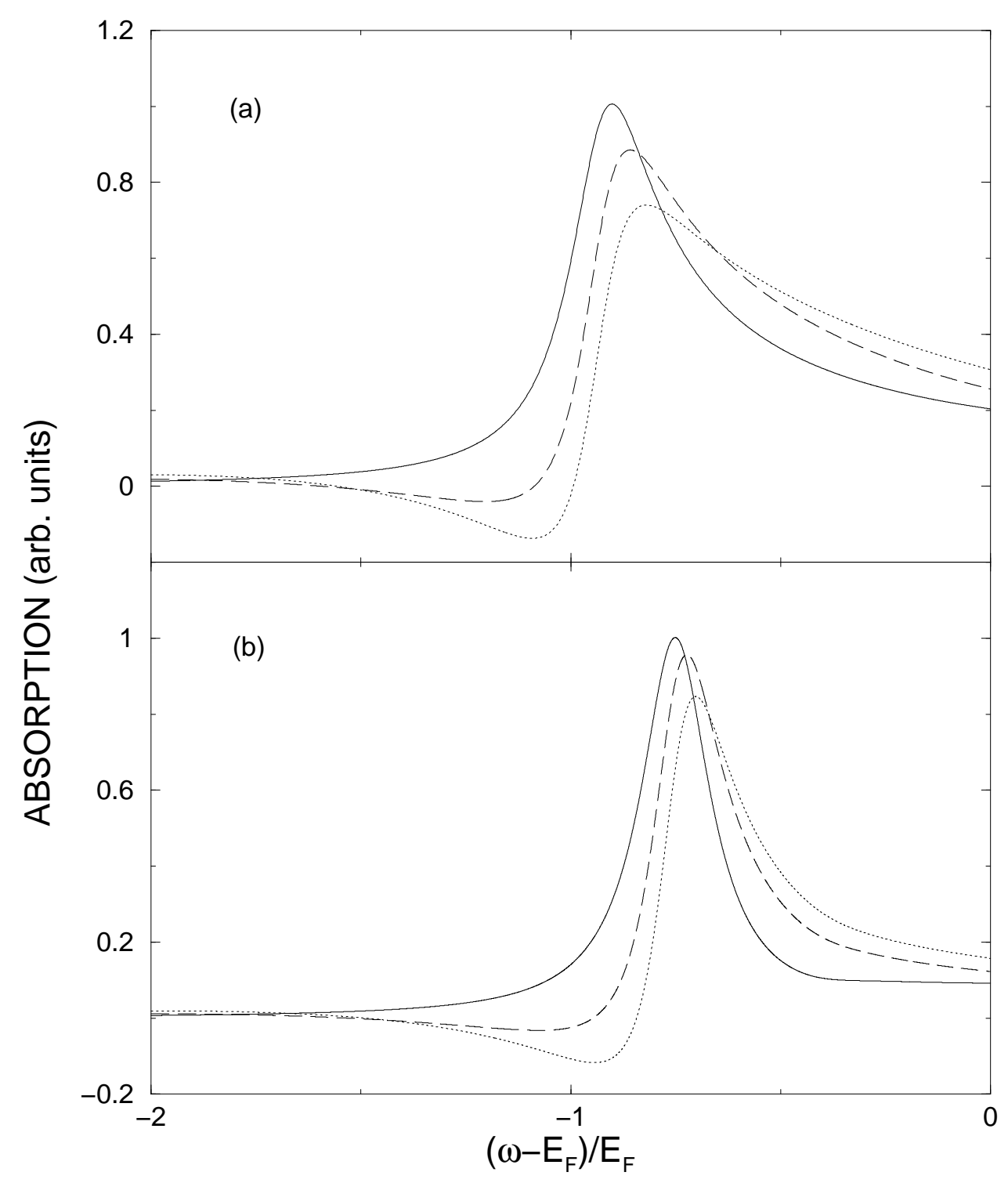

Figure 11: The effect of the pump-induced renormalization of the band dispersions on the resonance strength. The nonlinear absorption spectrum for the FES (a) compared to the HFA (b). Solid curves: Linear absorption. Dashed curves: Nonlinear absorption. Dotted curves: Nonlinear absorption for a rigid band shift only. The curves were calculated with $g=0.4, \Gamma=$ $0.1 E_{F}$, and $t_{p}=2.0 E_{F}^{-1}$. 


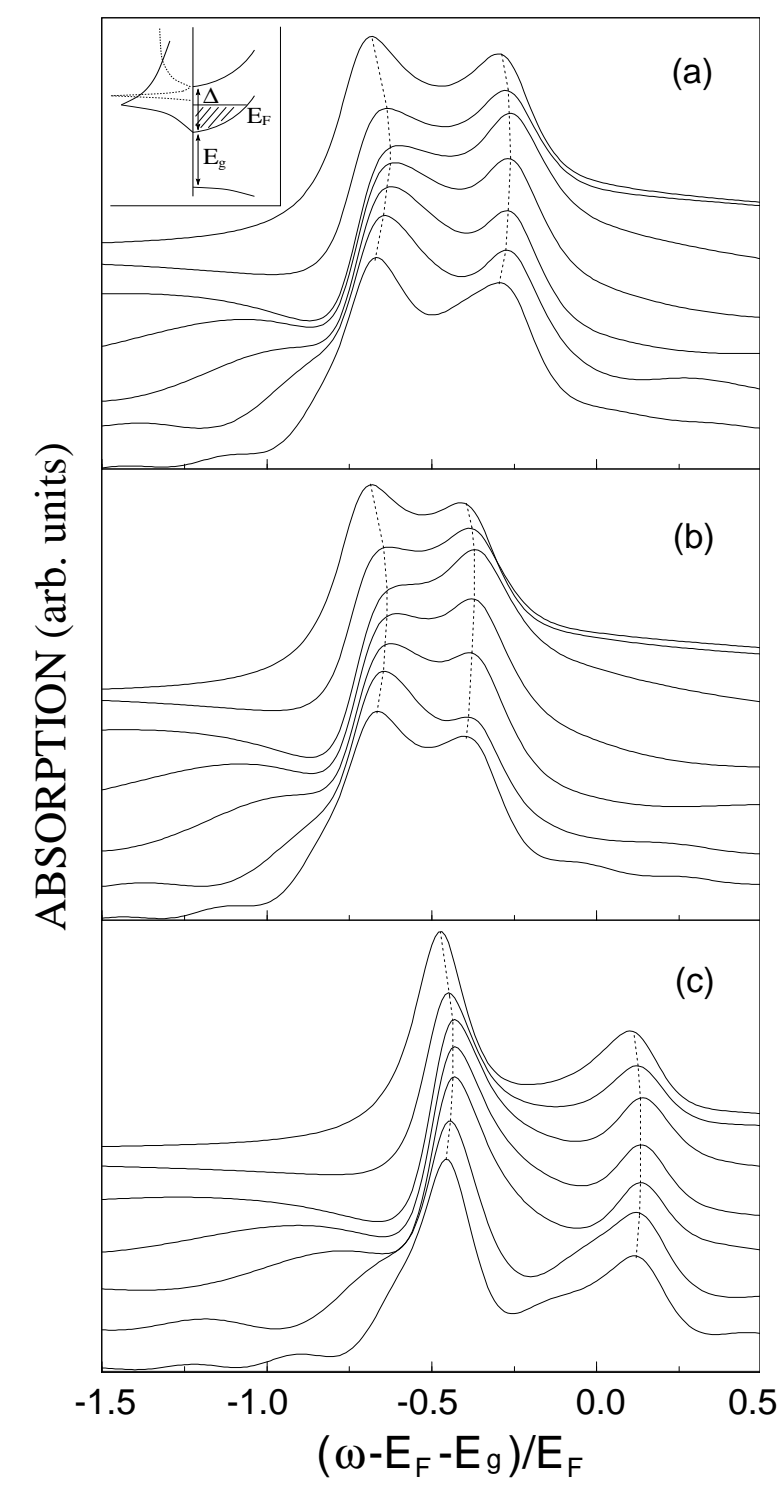

Figure 12: (a) Calculated PP spectra with (a) $\Delta=1.7 E_{F}$, (b) $\Delta=1.6 E_{F}$, and (c) $\Delta=1.6 E_{F}$ (HFA), for short pump duration $t_{p} E_{F} / \hbar=2.0$, and negative time delays $\tau \Gamma / \hbar=-2.0$ (lowest curve), $-1.2,-0.6,-0.4,-0.2,0$, and linear absorption spectrum (upper curve). Inset: schematic plot of the energy spectrum of the two-subband QW (right) and absorption spectrum (left). 

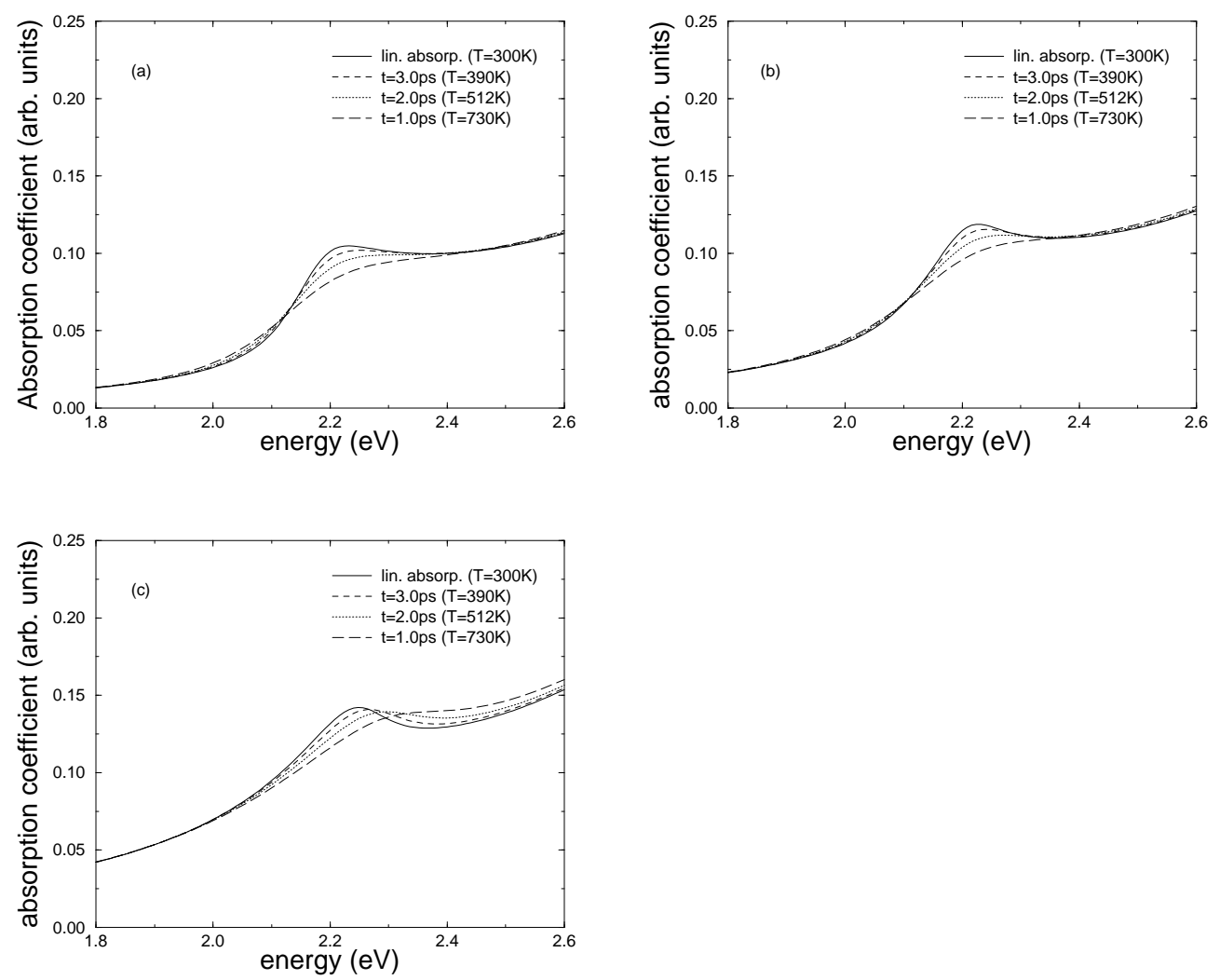

Figure 13: Calculated absorption spectra at positive time delays for nanoparticles with (a) $R=5 \mathrm{~nm}$, (b) $R=2.5 \mathrm{~nm}$, and (c) $R=1.2 \mathrm{~nm}$. 

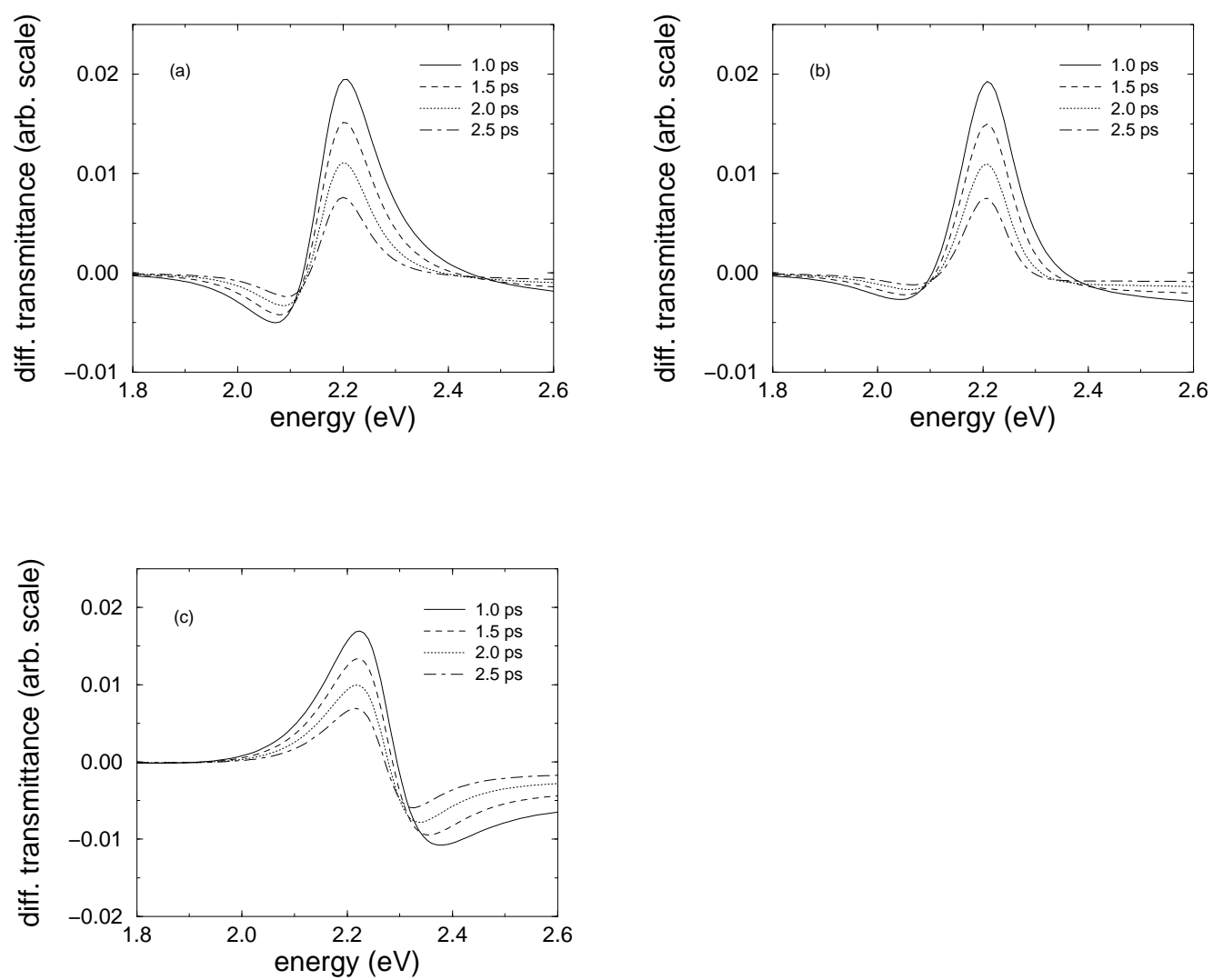

Figure 14: Calculated differential transmission spectra at positive time delays for nanoparticles with (a) $R=5 \mathrm{~nm}$, (b) $R=2.5 \mathrm{~nm}$, and (c) $R=1.2$ nm. 

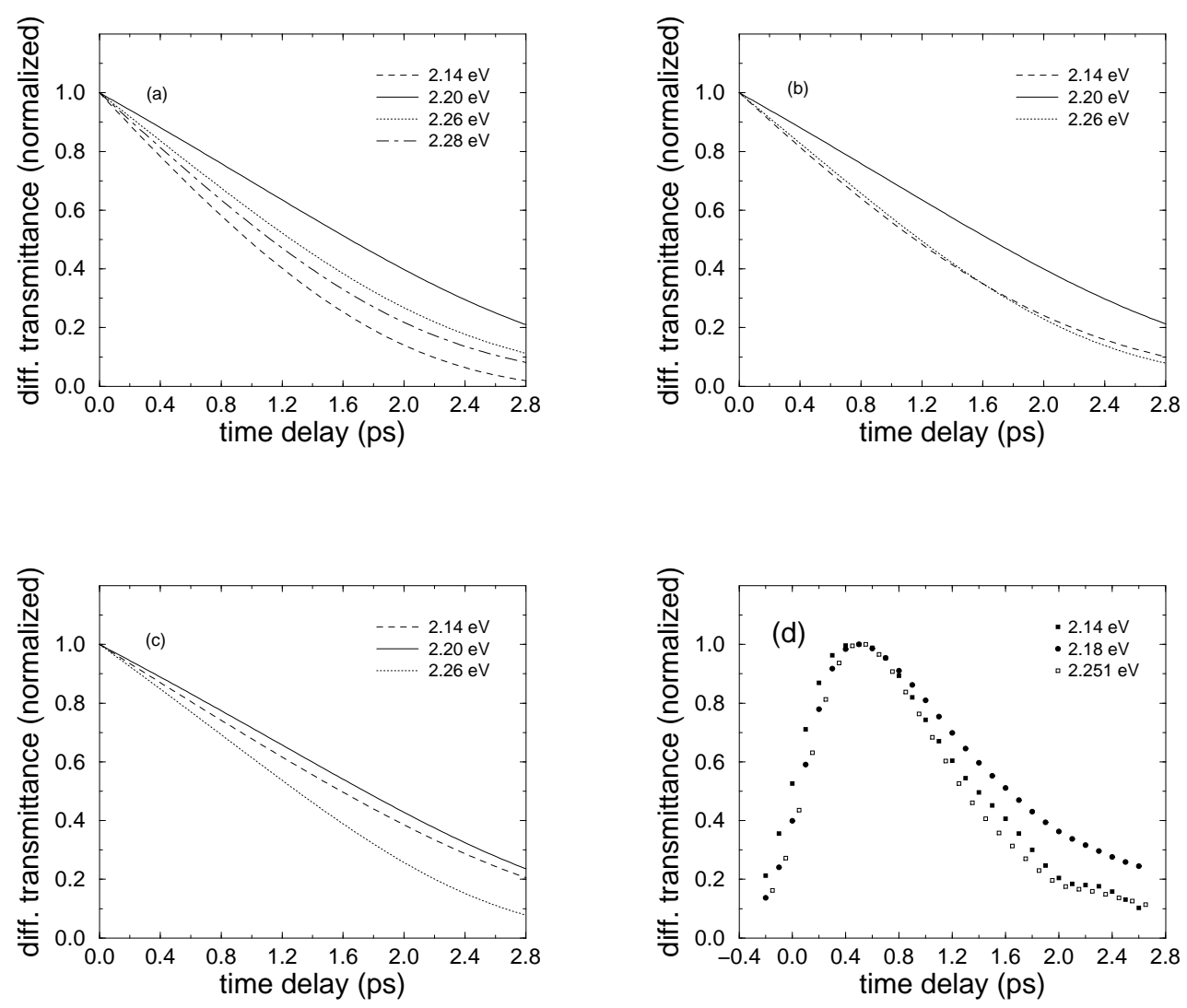

Figure 15: Temporal evolution of the differential transmission at frequencies close the SP resonance for nanoparticles with (a) $R=5 \mathrm{~nm}$, (b) $R=2.5 \mathrm{~nm}$, and (c) $R=1.2 \mathrm{~nm}$. (d) Measured time-resolved pump-probe signal. 